\title{
Design and Game-Theoretic Analysis of Community-Based Market Mechanisms in Heat
} and Electricity Systems

\author{
Mitridati, Lesia Marie-Jeanne Mariane; Kazempour, Jalal; Pinson, Pierre
}

Published in:

Omega: The International Journal of Management Science

Link to article, DOI:

10.1016/j.omega.2019.102177

Publication date:

2020

Document Version

Early version, also known as pre-print

Link back to DTU Orbit

Citation (APA):

Mitridati, L. M-J. M., Kazempour, J., \& Pinson, P. (2020). Design and Game-Theoretic Analysis of CommunityBased Market Mechanisms in Heat and Electricity Systems. Omega: The International Journal of Management Science, 99, [102177]. https://doi.org/10.1016/j.omega.2019.102177

\section{General rights}

Copyright and moral rights for the publications made accessible in the public portal are retained by the authors and/or other copyright owners and it is a condition of accessing publications that users recognise and abide by the legal requirements associated with these rights.

- Users may download and print one copy of any publication from the public portal for the purpose of private study or research.

- You may not further distribute the material or use it for any profit-making activity or commercial gain

- You may freely distribute the URL identifying the publication in the public portal 


\title{
Design and Game-Theoretic Analysis of Community-Based Market Mechanisms in Heat and Electricity Systems
}

\author{
Lesia Mitridati* \\ Georgia Institute of Technology, H. Milton Stewart School of Industrial \& Systems Engineering, 755 Ferst Dr NW, \\ Atlanta, GA 30318, USA \\ Jalal Kazempour, Pierre Pinson** \\ Technical University of Denmark, Department of Electrical Engineering, Elektrovej 325, DK-2800 Kongens Lyngby, \\ Denmark
}

\begin{abstract}
In an increasingly decentralized energy system with tight interdependencies with heat and electricity markets, prosumers, who act both as consumers and producers at the interface between the markets, are becoming important operational flexibility providers. Policy-makers and market operators need a better understanding of the motivations and behavior of prosumers in order to harness their underlying flexibility. In that context, existing market mechanisms must be accompanied by decision-making tools which enable direct participation of prosumers and cooperation among them towards a social choice. This work focuses on designing a community-based market mechanism, in which prosumers can pool their heat and electricity production and consumption, and coordinate their participation in heat and electricity wholesale markets. A central research question that is addressed, is to understand how this mechanism can affect the outcomes of the interactions among individuals towards a social choice, and in particular incentivize cooperation among prosumers. Game-theoretic concepts are used to analyze the properties of the proposed market mechanism with different allocation schemes, namely uniform pricing, Vickrey-Clarke-Groves, Shapley value, and nucleolus. This analysis shows that it is beneficial for the community as a whole to cooperate and that there exists a set of stable allocations for the proposed mechanism. Additionally, although no allocation can satisfy all fundamental desirable market properties, this study demonstrates that the proposed mechanism based on a nucleolus allocation can provide an interesting trade-off between stability, efficiency, and incentive compatibility. Finally, the concepts and properties discussed in this work are illustrated in a case study.
\end{abstract}

Keywords: Community-based market; Multi-carrier energy system; Mechanism design; Game theory

\footnotetext{
${ }^{*}$ Corresponding author. Email address lmitridati3@gatech.edu (L. Mitridati)

** This research was partially supported by the Danish Energy Development Programme (EUDP) through the EnergyLab Nordhavn project (64015-0055). Pierre Pinson was additionally supported by IF through CITIES project (DSF- 1305-00027B), while Jalal Kazempour and Lesia Mitridati were also supported by EUDP through the CORE project (64017-0005).
} 


\section{Introduction}

Worldwide, the energy transition towards a more environmentally friendly and sustainable energy system has lead to an unprecedented share of the generation mix being covered by renewable power production (Chu \& Majumdar, 2012; Lund, 2007; Chu et al., 2017). Managing these large shares of decentralized, stochastic, and non-dispatchable production has increased the need for operational flexibility ${ }^{1}$ in power systems (NERC, 2010; Morales et al., 2013).

In addition to the traditional sources of operational flexibility, such as dispatchable thermal power plants and electricity storage, flexibility in power systems may come from the interfacing and coordination with other energy systems, in particular the heat system (Lund, 2005; Lund et al., 2010, 2014; Meibom et al., 2013; Münster et al., 2012; Lund et al., 2015a; Wang et al., 2017c). At the wholesale level, harnessing this operational flexibility requires the design of new markets and trading mechanisms that model and exploit the interactions between the systems (Mitridati \& Pinson, 2016; Pinson et al., 2017).

In the meantime, a more profound and fundamental shift is happening in heat and electricity systems due to a change from a top-down energy system management approach to a decentralized approach (Bouffard \& Kirschen, 2008). Motivated by growing environmental concerns and social commitment (Goulden et al., 2014; Gangale et al., 2013), a new category of energy users, also called prosumers, who may consume, produce, store, and share energy, has emerged as central and proactive energy market participants. In particular, in dense urban areas, where electricity and district heating infrastructures cohabit, prosumers interface both energy systems. It is expected that harnessing their operational flexibility can induce a soft coordination between the systems and benefit the overall energy system, in terms of social welfare and sustainability (Wang et al., 2017b; Geidl et al., 2007; Lund et al., 2015b). Kirschen (2003) provides an overview of the potential benefits of better accounting for demand-side flexibility in future wholesale market designs. In addition, O'Connell et al. (2016), Liu et al. (2015), and Bobo et al. (2018) highlight the need for new trading mechanisms, such as flexibility-oriented products and extended bid formats, that facilitate market access to prosumers, and reveal the value of their operational flexibility in a market environment. Additionally, policy-makers and market operators lack a better understanding of the motivations and behavior of prosumers to effectively and efficiently integrate them into electricity markets (Stern, 1992; Allcott \& Mullainathan, 2010; Gyamfi et al., 2013; Da Silva et al., 2012; Lampropoulos et al., 2010). In that context, the aforementioned top-down market solutions should be accompanied by consumer-centric decision-making tools, which enable prosumers to directly manage their energy consumption and production. Parag \& Sovacool (2016) and Sousa et al. (2019) identify and discuss different potential consumer-centric market mechanisms. By opposition with top-down demand-response programs, consumer-centric market mechanisms focus on motivating direct participation from the prosumers, and provide a framework that coordinates the outcomes of interactions among them towards a social choice (Haring et al., 2016). The motivation behind this approach is to take advantage of under-utilized sources of flexibility and synergies among prosumers, not unlike the concept of sharing-economy in other industry sectors (Kalathil et al., 2017; Heinrichs, 2013). Kalkbrenner \& Roosen (2016) and Klein \& Coffey (2016) highlight how local energy communities can increase social acceptance and motivation to participate in demand response programs, compared to traditional top-down approaches.

\footnotetext{
${ }^{1}$ The term operational flexibility, or simply flexibility, is traditionally defined as the capability of a system to modify its output or state in response to a signal (Zhao et al., 2016).
} 
The increasingly decentralized energy systems highlight the need for consumer-centric market mechanisms aiming at harnessing the flexibility of prosumers across multiple energy carriers, namely heat and electricity. The design and analysis of such mechanisms raise two conceptual research questions.

First, what value can a group of prosumers achieve by exploiting the flexibility and synergies of different types of loads? The term of value is understood in a game-theoretic context as the increase in social welfare that a group of prosumers can achieve by cooperating rather than standing alone (Peters, 2015). This value represents the benefits for the community as a whole when prosumers collaborate. In a multi-carrier energy system, this value arises from exploiting the flexibility of prosumers and the synergies among different types of loads, as illustrated in Figure 1.

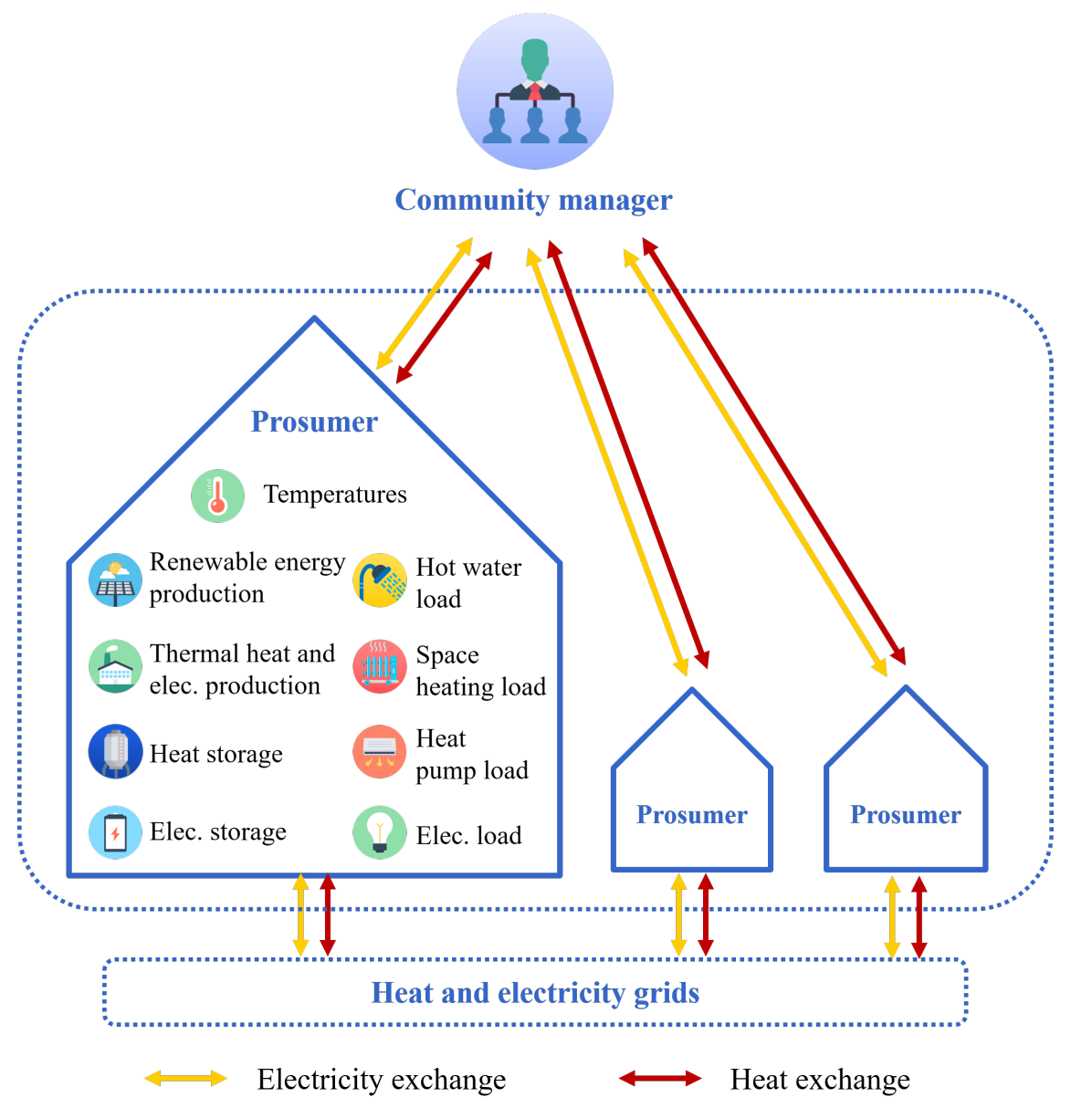

Figure 1: Detailed model of an individual prosumer in the energy community.

In particular, fuel-shift technologies, such as heat pumps (HPs) and micro combined heat and power plants (CHPs), in heat and electricity markets have been shown to be important sources of cross-carrier flexibility (Wang et al., 2017a; Alimohammadisagvand et al., 2016; De Paepe et al., 2006; Houwing et al., 2011). In addition, modeling and exploiting the thermal storage capacity of buildings greatly increase the operational flexibility of prosumers and their ability to provide demand response in both heat and electricity systems (Good \& Mancarella, 2017; Alimohammadisagvand et al., 2016; Dominković et al., 2018). From an economic perspective, valuing the flexibility 
of different types of loads, such as district heating and electricity consumption of heat pumps, requires modeling their synergies. Various studies have proposed community-based control strategies for the energy management of building that model these technical and economic aspects (Brahman et al., 2015; Good \& Mancarella, 2017; Wang et al., 2018; Darivianakis et al., 2015, 2017b). In particular, Shaikh et al. (2014) and Klein et al. (2012) propose control strategies for buildings based on the utility that prosumers derive from the indoor temperature, rather than the energy carrier consumed. These operational models highlight the potential benefits of cooperating and provide operational models to achieve that value (Alam et al., 2013; Wang et al., 2017a). In practice, the potential for exploiting the flexibility from heat and electricity prosumers at a local level is significant. The authors in Brange et al. (2016) carried out a techno-economic analysis of the impact of heat and electricity prosumers in Malmö, Sweden, and showed that excess heat could cover $50 \%$ to $120 \%$ of the heat demand in this neighborhood. Additionally, various research and demonstration projects have tackled the issue of the integration of heat and electricity systems at a local level in order to create net-zero emission neighborhoods, such as the Energylab Nordhavn project (Denmark) and Östra Sala (Sweden) projects Kılkış (2015); Energylab Nordhavn project (2019). As highlighted in the recommendations of the Energylab Nordhavn project, the focus should be placed on exploiting the flexibility of energy consumers through new market-based mechanisms Energylab Nordhavn project (2019). This raises a second conceptual question.

How to design a consumer-centric market mechanism that incentivizes prosumers to collaborate towards this social optimum? An efficient community-based market mechanism should aim at coordinating the energy production and consumption of a group of prosumers towards a social optimum, i.e., a maximum social welfare for the community as a whole. Achieving this value in a market environment requires designing institutions and trading mechanisms that accurately model the physical and economic characteristics of each agent, and facilitate interactions between them. However, commonly-used bid formats, such as price-quantity and block bids, are not adequate to accurately model the strong dependencies across multiple time steps and energy carriers. Various studies have suggested that extending standard price-quantity bids could help reveal the flexibility of prosumers (Liu et al., 2015). Yet, no such a bid format has been proposed for multi-carrier energy systems. Furthermore, a consumer-centric approach must ensure that prosumers are voluntarily participating in the proposed mechanism, and are satisfied with the outcomes. Such a mechanism is called stable. Beyond the concept of value for the community as a whole, this raises the issue of the fair allocation of this value among the prosumers. Addressing this question requires modeling the outcomes of this mechanism, as well as the behavior and interactions among the agents through game-theoretic models (Roth, 2000). A series of recent studies have adopted a consumer-centric approach for prosumers in electricity markets (Morstyn et al., 2018; Sorin et al., 2019; Haring et al., 2016; Moret \& Pinson, 2019), but has failed to provide a thorough analysis of the properties of these mechanisms. Han et al. (2018) and Tushar et al. (2018) propose a cooperative gametheoretic analysis of the efficiency and fairness of a community-based market mechanism for energy management. However, these studies assume complete information on the preferences of each agent, which is quite restrictive. Ensuring the truthful participation of agents with privately known preferences, i.e., incentive compatibility, is a central research question in the field of mechanism design (Hurwicz, 1972). However, a gap remains in analyzing the link and trade-off between incentive compatibility, efficiency, and stability of consumer-centric market mechanisms.

The main objective of this work is to design a consumer-centric market mechanism that incentivizes prosumers in a multi-carrier energy system to collaborate to optimize the energy man- 
agement of the community. In view of the existing literature, this work makes several applied and theoretical contributions to the state of the art. From an applied perspective, we design a novel community-based market mechanism, in which prosumers can pool their heat and electricity production and consumption, and coordinate their participation in heat and electricity wholesale markets. The proposed market mechanism provides adequate institutions and trading mechanisms to reflect the physical and economic aspects of the flexibility of prosumers at the interface between heat and electricity systems. From a theoretical perspective, we demonstrate two fundamental properties of the proposed community-based market mechanism through game-theoretic models. We first demonstrate that there exists a non-empty set of stabilizing allocations for the proposed mechanism. We then demonstrate that the particular structure of the proposed mechanism provides an interesting trade-off between the properties of incentive compatibility, efficiency, and stability. Finally, we analyze the properties of this community-based market mechanism for different allocation mechanisms, namely uniform pricing, Vickrey-Clarke-Groves (VCG), Shapley value, and nucleolus. While it is known that, among these allocations, solely the nucleolus allocation provides a stabilizing mechanism in the general case, we demonstrate that, under the assumption of perfect competition among the prosumers, the uniform pricing allocation is also stabilizing.

The remainder of this paper is organized as follows. Section 2 introduces the general framework and theoretical background for the design and analysis of consumer-centric market mechanisms. Section 3 presents in details the proposed mechanism, along with different allocation mechanisms. Section 4 analyzes the properties of the resulting mechanisms. Section 5 illustrates the theoretical findings of this work using a case study. Finally, Section 6 presents the conclusions and discussions.

\section{Status quo of consumer-centric market mechanism design}

This section presents an overview of the literature on consumer-centric market mechanisms for energy management and lays the foundation for the proposed approach. Additionally, for the sake of self-containment, general concepts related to the field of mechanism design, and game-theoretic models are presented in order to formulate and analyze the properties of the proposed mechanism.

\subsection{Consumer-centric market mechanisms in energy systems}

Traditionally, the operational flexibility of residential prosumers is being harnessed by entities, the so-called aggregators, who are profit-maximizers, and centrally manage a group of loads via direct control (Wang \& Deng, 2019) or indirect price signals (Callaway \& Hiskens, 2011). Koch \& Piette (2009) provide an overview of the relative benefits of direct and indirect load control programs. While these approaches are designed to provide incentives and elicit demand response from prosumers, they still suffer from various economic, regulatory, technological, and social barrier (O'Connell et al., 2014; Vallés et al., 2016; Good et al., 2017; Medina et al., 2010). In particular, these centralized mechanisms for demand response fail to motivate cooperation among prosumers, and exploit the synergies across different types of prosumers and load (Haring et al., 2016).

Contrary to centralized demand response mechanisms, in which a profit-maximizing aggregator controls a group of loads via prices or direct signals, decentralized mechanisms, also referred to as consumer-centric approaches, provide a framework in which consumers act cooperatively to exchange energy, and aggregate their flexibility (Haring et al., 2016). Parag \& Sovacool (2016) provide an overview of different consumer-centric market mechanisms. Consumer-centric approaches can broadly be divided into peer-to-peer and community-based mechanisms, as illustrated in Figure 2 . In a peer-to-peer mechanism, prosumers communicate directly with one another and trade 
their energy via bilateral contracts and agreements (Morstyn et al., 2019). This approach allows energy to be treated as a heterogeneous product with certain attributes, on which prosumers can express their preferences (Morstyn \& McCulloch, 2019). However, this approach raises issues of communication and convergence towards a social choice. By opposition, in community-based market mechanisms, the information $\mathcal{B}_{j}$ from all prosumers $j \in \mathcal{N}$ is gathered by a so-called community manager. Based on the revealed information $\mathcal{B}=\left[\mathcal{B}_{1}, \ldots, \mathcal{B}_{N}\right]$, the community manager seeks to maximize the social welfare of the community, i.e., the sum of the individual utilities of all agents (Moret et al., 2018; Morstyn et al., 2018; Sorin et al., 2019). Once an optimal strategy for the community has been identified $X^{\mathcal{N}^{*}}(\mathcal{B})=\left[X_{1}^{\mathcal{N}^{*}}(\mathcal{B}), . ., X_{N}^{\mathcal{N}^{*}}(\mathcal{B})\right]$, the community manager computes the value of this cooperation and derives a payment rule $\mathcal{P}\left(X^{\mathcal{N}^{*}}(\mathcal{B})\right)$ that redistributes this value among the prosumers.

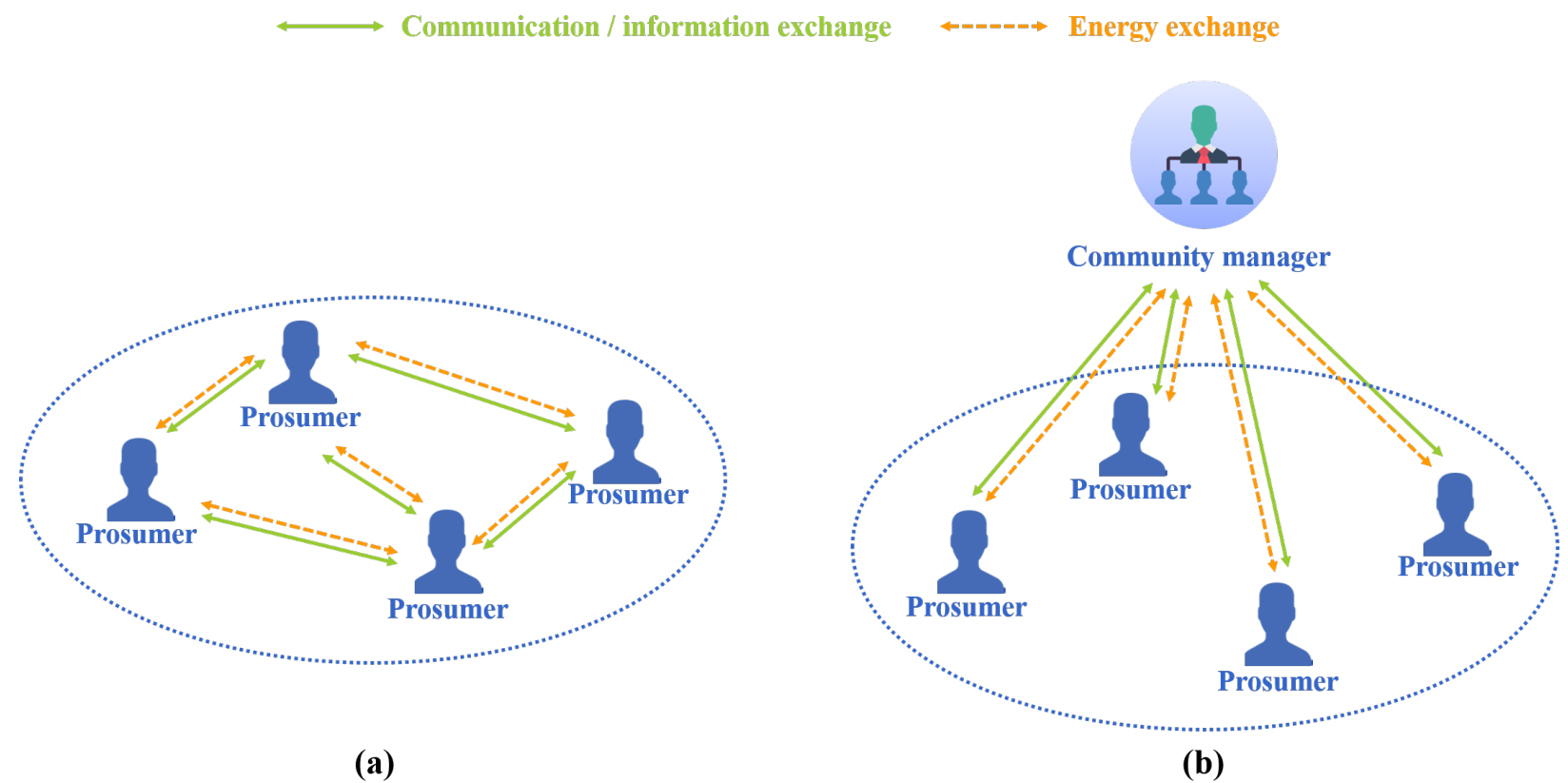

Figure 2: Organization of consumer-centric market mechanisms: (a) peer-to-peer, and (b) community-based.

At the community level, adequately-designed community-based energy markets incentivize prosumers to cooperate in order to achieve a social choice. At a societal level, community-based market design facilitates the coordination of power and heat systems at the level of end-users, and enable flexibility to be exchanged locally. This approach empowers individual end-users to exploit their own flexibility locally, and eventually facilitates the integration of end-users to the market. The flexibility unlocked by the local coordination will open new opportunities to further integrate renewables in energy systems. In order to achieve these individual and societal benefits, adequate market mechanisms should guarantee certain desirable properties. These properties are introduced below in the theoretical framework provided by the fields of mechanism design and game theory.

\subsection{Mechanism design and desirable properties}

The field of mechanism design provides a framework to design rules and institutions that coordinate the interactions between self-interested agents, and analyze the outcomes of these interactions. 
The interested reader is referred to Hurwicz (1973), Silva et al. (2001), Vulkan et al. (2013) and Hurwicz \& Reiter (2006) for an introduction to the theory of mechanism design. The central question that must be addressed when designing any mechanism, is how the proposed rules and institutions impact the interactions among the agents. In other words, what properties does the proposed mechanism satisfy? The following describes a number of desirable properties, analyzed in the context of a community-based market mechanism with decentralized information.

As by design the proposed consumer-centric market mechanism seeks to maximize the true social welfare of the energy community, it should satisfy the fundamental efficiency property ${ }^{2}$.

Property 1 (Efficiency). The social welfare of the agents participating in the mechanism, with respect to the revealed preferences $\mathcal{B}$, is maximized, and no agent desires to deviate unilaterally from its dispatch. In other words, the outcomes of social welfare maximization align with profitmaximization of agents.

This property is essential to guarantee that the community as a whole, benefits from cooperating. Indeed, it guarantees that the outcomes of social welfare maximization align with the maximization of the private utilities of the agents. Furthermore, the true social welfare of the community is maximized, and the true value of cooperating is achieved in case the agents are truthful, i.e., reveal their true preferences. As the agents hold private information, they might have incentives to misreport their true preferences in order to manipulate the outcomes of the community management problem and increase their profits, or for privacy concerns. Thus, designing a mechanism that motivates the agents to be truthful is a major challenge for the community manager. Such a property is referred to as incentive compatibility.

Property 2 (Incentive compatibility). Every agent can maximize its utility by acting according to its true preferences.

Furthermore, in order to ensure social acceptance, a consumer-centric market mechanism should ensure that the outcomes of the mechanism are considered fair by all agents. Various definitions of fairness in mechanism design have been used in the literature. In this study, it is used in the sense of a stable allocation mechanism that ensures that all agents are satisfied with the outcomes of the mechanism and do not wish to split from the community. Tushar et al. (2018) link the social acceptance of a consumer-centric market mechanism to this stability property using behavioral economy theory. For that purpose, two essential properties are budget balance and group rationality.

Property 3 (Budget balance (allocation efficiency)). The community manager has neither financial deficit nor financial excess.

This property ensures that the community manager redistributes the entire benefits, or losses, among the prosumers. In this context, it is particularly important as it guarantees that the community manager is non-profit.

Property 4 (Group rationality). No group of agents can benefit by splitting from the community.

\footnotetext{
${ }^{2}$ Note that the concept of efficiency is defined with respect to the revealed preferences rather than the true preferences.
} 
As no individual agent or group of agents can benefit by splitting from the community, this property guarantees that the outcomes of the mechanism are stable. These two properties will be formally defined in Section 2.3, within the conceptual framework of cooperative game theory.

A mechanism satisfying Properties 1 - 4 ensures that the private utilities of the agents are aggregated to achieve a social choice. In the context of the proposed community-based market mechanism, these properties incentivize prosumers to collaborate to maximize the social welfare of the community. An in-depth analysis of these properties for different mechanisms will be provided in Section 4. However, a fundamental result states that no mechanism with decentralized information can guarantee simultaneously efficiency, incentive compatibility, budget balance, as well as cost recovery for all agents (Hurwicz, 1972; Myerson \& Satterthwaite, 1983). Similarly, Roth (1982) states that no mechanism can guarantee incentive compatibility, efficiency, budget balance, and group rationality. Thus, a major design challenge which is addressed in this study is to identify the links and the trade-off between these properties.

\subsection{Game-theoretic tools}

Analyzing the properties of a given mechanism requires modeling the outcomes of this mechanism, as well as the behavior of the agents and the interactions between them. The literature on mechanism design relies on cooperative and non-cooperative game-theoretic tools to do so (Roth, 2000). The main distinction between cooperative and non-cooperative game theory is in the modeling technique that is used. Non-cooperative game theory provides an explicit model of the behavior and interactions among the agents, whereas cooperative game theory provides an abstract representation of the agents through the outcomes and payoffs that they can reach (Peters, 2015; Saad et al., 2009; Osborne \& Rubinstein, 1994; Nisan et al., 2007). Although cooperative and non-cooperative game-theoretic tools rely on different modeling techniques, they are both used to answer the same central question, i.e., what is the impact of the institutions and rules imposed by a mechanism on the behavior of the agents and interactions between them? Therefore, this section revisits the properties introduced in Section 2.2 under both cooperative and non-cooperative game-theoretic frameworks.

\subsubsection{Cooperative game theory}

The starting point of a cooperative game-theoretic analysis of a mechanism is to define the value $v^{\mathcal{S}}(\mathcal{B})$ with respect to the revealed preferences $\mathcal{B}$ that can be achieved by a group of agents forming a coalition $\mathcal{S} \in 2^{\mathcal{N}}$, where $2^{\mathcal{N}}$ represents the set of all coalitions included in the grand-coalition $\mathcal{N}$. In particular, in the community-based market mechanism considered, the value of a coalition is defined as the increase in social welfare, compared to the case where all agents in the coalition optimize their energy management strategy alone. The coalitional game form of a mechanism is defined as follows.

Definition 1 (Coalitional game form). The pair $(\mathcal{N}, v(\mathcal{B}))$ defines a cooperative game if and only if the value function $v(\mathcal{B}): \mathcal{S} \in 2^{\mathcal{N}} \mapsto v^{\mathcal{S}}(\mathcal{B}) \in \mathbb{R}^{+}$satisfies

1. $v^{\emptyset}(\mathcal{B})=0$, i.e., the value of an empty coalition is null.

2. For any two selected coalitions $\mathcal{S} \in 2^{\mathcal{N}}, \mathcal{V} \in 2^{\mathcal{N}}$, such that $\mathcal{S} \cap \mathcal{V}=\emptyset$ super-additivity holds, i.e., $v^{\mathcal{S}}(\mathcal{B})+v^{\mathcal{V}}(\mathcal{B}) \leq v^{\mathcal{S} \cup \mathcal{V}}(\mathcal{B})$.

Super-additivity implies that the value of any coalition cannot be improved by splitting it into smaller coalitions. In practice, this guarantees that overall it is beneficial to form the grandcoalition $\mathcal{N}$, rather than collaborating in smaller coalitions $\mathcal{S} \in 2^{\mathcal{N}}$, or standing alone. However, 
it does not guarantee that all agents benefit individually from joining the grand-coalition, and are satisfied with the outcomes of the proposed mechanism. This raises the issue of a fair redistribution of this value through the design of an allocation mechanism. The allocation mechanism of a cooperative game can be directly expressed as a function of the payment rule of the corresponding mechanism. Therefore, the notions of allocation and payment, though different, can be used equivalently to describe the outcomes of a mechanism and its properties. The vector of allocations $\pi\left(X^{\mathcal{N}^{*}}(\mathcal{B})\right) \in \mathbb{R}^{N}$, whose entries $\pi_{j}\left(X^{\mathcal{N}^{*}}(\mathcal{B})\right)$ represent the share of the value $v^{\mathcal{N}}(\mathcal{B})$ allocated to an agent $j \in \mathcal{N}$.

In this cooperative game-theoretic framework, the budget balance (or efficient allocation) property of the proposed community-based market mechanism introduced in Property 3 rewrites as

$$
\sum_{j \in \mathcal{N}} \pi_{j}\left(X^{\mathcal{N}^{*}}(\mathcal{B})\right)=v^{\mathcal{N}}(\mathcal{B})
$$

This ensures that the entire value of the coalition, i.e., the increase in social welfare, is allocated to the agents. Additionally, in order to measure the satisfaction of agents in the grand-coalition $\mathcal{N}$ with respect to an allocation $\pi\left(X^{\mathcal{N}^{*}}(\mathcal{B})\right)$, we define the excess of a coalition as follows.

Definition 2 (Excess). The excess $\epsilon\left(\pi\left(X^{\mathcal{N}^{*}}(\mathcal{B})\right)\right) \in \mathbb{R}^{2^{N}-2}$ is a vector whose entries

$$
\epsilon_{\mathcal{S}}\left(\pi\left(X^{\mathcal{N}^{*}}(\mathcal{B})\right)\right)=v^{\mathcal{S}}(\mathcal{B})-\sum_{j \in \mathcal{S}} \pi_{j}\left(X^{\mathcal{N}^{*}}(\mathcal{B})\right)
$$

represent the dissatisfaction of the coalition $\mathcal{S} \in 2^{\mathcal{N}} \backslash\{\mathcal{N}, \emptyset\}$ with respect to the allocation $\pi\left(X^{\mathcal{N}^{*}}(\mathcal{B})\right)$, arranged in decreasing order.

By construction, the excess of the grand-coalition or an empty coalition would be equal to zero for any efficient allocation $\pi\left(X^{\mathcal{N}^{*}}(\mathcal{B})\right) \in \mathcal{I}(v(\mathcal{B}))$, but this is not necessarily true for other coalitions $\mathcal{S} \in 2^{\mathcal{N}} \backslash\{\mathcal{N}, \emptyset\}$. In fact, if $\epsilon_{\mathcal{S}}\left(\pi\left(X^{\mathcal{N}^{*}}(\mathcal{B})\right)\right)>0$, the agents in the coalition $\mathcal{S} \in 2^{\mathcal{N}} \backslash\{\mathcal{N}, \emptyset\}$ can achieve higher profits by leaving the grand-coalition $\mathcal{N}$. In that context, a cooperative gametheoretic interpretation of the group rationality property is the set of allocations that guarantee non-positive excesses for all coalitions $\mathcal{S} \in 2^{\mathcal{N}} \backslash\{\mathcal{N}, \emptyset\}$. Let us define the revealed core $\mathcal{C}(\mathcal{N}, v(\mathcal{B}))$ of the cooperative game $\mathcal{N}, v(\mathcal{B})$ as the set of efficient allocation mechanisms that respect group rationality, i.e.,

Definition 3 (Revealed core). The revealed core $\mathcal{C}(\mathcal{N}, v(\mathcal{B}))$ of a cooperative game is defined as the set of allocations such that no coalition $\mathcal{S} \in 2^{\mathcal{N}} \backslash\{\mathcal{N}, \emptyset\}$ has a positive excess with respect to any of those allocations, i.e., $\mathcal{C}(\mathcal{N}, v(\mathcal{B}))=\left\{\pi\left(X^{\mathcal{N}^{*}}(\mathcal{B})\right) \in \mathcal{I}(v(\mathcal{B})) \mid \epsilon_{\mathcal{S}}\left(\pi\left(X^{\mathcal{N}^{*}}(\mathcal{B})\right)\right) \leq 0, \quad \forall \mathcal{S} \in\right.$ $\left.2^{\mathcal{N}} \backslash\{\mathcal{N}, \emptyset\}\right\}$.

Thus, a so-called core-selecting mechanism that systematically selects an allocation that is in the revealed core of the cooperative game is considered fair by the agents participating in this mechanism, and is referred to as a stabilizing mechanism (Day \& Milgrom, 2008).

Definition 4 (Core-selecting mechanism). A core-selecting mechanism selects, for any revealed preferences $\mathcal{B}$, a dispatch $X^{\mathcal{N}^{*}}(\mathcal{B})$ and an allocation $\pi\left(X^{\mathcal{N}^{*}}(\mathcal{B})\right)$ in the revealed core $\mathcal{C}(\mathcal{N}, v(\mathcal{B}))$ of the cooperative game $(\mathcal{N}, v(\mathcal{B}))$.

Note that, the main concepts of cooperative game theory are defined with respect to the revealed preferences $\mathcal{B}$. However, cooperative game-theoretic tools do not provide an explicit model of the decisions of individual agents. Therefore, non-cooperative game theory can expand the scope of this analysis and provide a framework to study the incentive compatibility of the proposed mechanism. 


\subsubsection{Non-cooperative game theory}

Non-cooperative game theory provides tools to analyze the decisions and interactions among the agents in a mechanism. In a game-theoretic framework, the vector of revealed preferences $\mathcal{B}_{j}$ of any agent $j \in \mathcal{N}$ is also referred to as its strategy. The most commonly used concept in non-cooperative game theory is the Nash equilibrium, which guarantees that the strategy $\mathcal{B}_{j}$ of each agent $j \in \mathcal{N}$ is optimal with respect to the strategies $\mathcal{B}_{-j}$ of the other agents $k \in \mathcal{N} \backslash\{j\}$, i.e., no agent can benefit from unilaterally deviating from the equilibrium outcome (Maskin, 1999). Therefore, the efficiency property can be interpreted as the existence of a Nash equilibrium that coincides with the maximum social welfare, i.e., a Pareto efficient Nash equilibrium.

A stronger concept is the dominant-strategy equilibrium, which guarantees that the strategy of each agent is optimal, regardless of the strategies of the other agents. Any dominant-strategy equilibrium is a Nash equilibrium. The incentive compatibility property, also called dominant-strategy incentive compatibility, requires that there exists a truth-revealing dominant-strategy equilibrium, i.e., each agent can achieve its optimal objective, regardless of what the others do, by revealing its true preferences. In other words, this guarantees that no agent can benefit from manipulating the outcomes of the mechanism by misreporting its true preferences. Therefore, this property is central in mechanisms with decentralized information.

\subsubsection{Link between stability, efficiency, and incentive compatibility}

The notion of revealed core in cooperative game theory has a clear interpretation in noncooperative game theory and equilibrium. Karaca et al. (2019) show that a mechanism is coalitionproof, in the sense that it prevents shill bidding, i.e., bidding under multiple identities, and collusion, if and only if it is a core-selecting mechanism. In other words, the notion of revealed core is directly linked to the coalition-proof Nash equilibrium concept introduced in Bernheim et al. (1987) and Bernheim \& Whinston (1987), which ensures that a Nash equilibrium is immune not only to individual deviations but also to self-enforcing coalitional deviations.

Furthermore, while it is known that there exists no dominant-strategy incentive compatible, efficient, and stabilizing mechanism (Roth, 1982), Day \& Milgrom (2008) investigate the trade-off between these three properties by introducing a weaker version of the dominant-strategy incentive compatibility property, called incentive optimality, which minimizes incentives to deviate from a truth-revealing strategy.

Definition 5 (Incentive profile). The incentive profile $\mu^{\pi}(\mathcal{B}) \in \mathbb{R}^{N}$ of a core-selecting mechanism $\left(X^{\mathcal{N}^{*}}(\mathcal{B}), \pi\left(X^{\mathcal{N}^{*}}(\mathcal{B})\right)\right)$ at the revealed preferences $\mathcal{B}$, defines the maximum gain for each agent $j \in$ $\mathcal{N}$ from unilaterally deviating from this strategy, i.e., $\mu_{j}^{\pi}(\mathcal{B})=\sup _{\hat{\mathcal{B}}_{j}} \pi_{j}\left(X^{\mathcal{N}^{*}}\left(\hat{\mathcal{B}}_{j}, \mathcal{B}_{-j}\right)\right), \quad \forall j \in$ $\mathcal{N}$.

Property 5 (Incentive optimality). A core-selecting mechanism $(X, p)$ provides optimal incentives at the revealed preferences $\mathcal{B}$ if there exists no other core-selecting mechanism $(X, \hat{p})$ that has a lower incentive profile at $\mathcal{B}$, i.e., $\mu_{j}^{\hat{\pi}}(\mathcal{B}) \leq \mu_{j}^{\pi}(\mathcal{B}), \quad \forall j \in \mathcal{N}$, with a strict inequality for at least one agent $j \in \mathcal{N}$. In particular, a core-selecting mechanism is incentive-optimal if it provides optimal incentives at any revealed preferences $\mathcal{B}$.

A fundamental theorem introduced by Day \& Milgrom (2008) states that a core-selecting mechanism is incentive-optimal if and only if for any revealed preferences $\mathcal{B}$ it chooses an allocation $\pi\left(X^{\mathcal{N}^{*}}(\mathcal{B})\right)$ that is Pareto efficient for the agents. In other words, if there is no other allocation 
in the revealed core $\hat{\pi}(\mathcal{B}) \in \mathcal{C}(\mathcal{N}, v(\mathcal{B}))$ that dominates it, i.e., $\hat{\pi}_{j}(\mathcal{B}) \geq \pi_{j}\left(X^{\mathcal{N}^{*}}(\mathcal{B})\right), \quad \forall j \in \mathcal{N}$ with strict inequality for at least one prosumer.

\section{Proposed community-based market mechanism for energy management in heat and electricity systems}

This section introduces the problem statement and details different steps of the proposed mechanism. First, the individual agents, i.e., prosumers in the community, and their private information $\mathcal{B}$ are described. We then introduce the mathematical formulation of the optimal energy management problem solved by the community manager. Finally, different payment rules and allocation mechanisms are presented. For brevity, this section introduces a compact formulation of these models.

A description of the optimization variables and preference matrices, all well as their dimensions, are provided in Appendix A. The mathematical models representing the operating constraints of the prosumers, and the optimization problem of the community manager are detailed in Appendix B.

\subsection{Problem statement}

This study aims at designing a market mechanism which incentivizes prosumers at the interface between electricity and district heating systems to truthfully cooperate in order to achieve a social choice. Based on the market design literature presented in Section 2.1, we propose a communitybased market mechanism, in which prosumers can pool their heat and electricity production and consumption, and coordinate their participation in heat and electricity wholesale markets.

The community manager in this mechanism complies with the following three general steps:

1. Elicit information from individual agents, i.e., prosumers in the community, on their preferences, represented by the matrix $\mathcal{B}=\left[\mathcal{B}_{1}, \ldots, \mathcal{B}_{N}\right] \in \mathbb{R}^{K_{1} \times N}$, where each column $\mathcal{B}_{j} \in \mathbb{R}^{K_{1}}$ represents the vector of preferences of a prosumer $j \in \mathcal{N}$. For notational simplicity, dimension $K_{1}$ represents the length of the vector of preferences of the prosumers, which includes their load utilities, production costs, and technical parameters of their building and assets over all hours $t \in \mathcal{T}$ of the day. In a market environment this information is elicited through the bids submitted by the individual agents, i.e., prosumers, to the market operator, i.e., community manager.

2. Determine the optimal energy dispatch and procurement strategy $X^{\mathcal{N}^{*}}(\mathcal{B}) \in \mathbb{R}^{K_{2} \times N}$ that maximizes the social welfare of the community with respect to the revealed preferences $\mathcal{B}$, where each column $X_{j}^{\mathcal{N}^{*}}(\mathcal{B}) \in \mathbb{R}^{K_{2}}$ represents the vector of optimal decisions of a prosumer $j \in \mathcal{N}$. For notational simplicity, dimension $K_{2}$ represents the length of the vector of decision variables of the prosumers, which includes their energy dispatch, exchanges with the grid and within the community, and state of their system over all hours $t \in \mathcal{T}$ of the day.

3. Given the optimal energy management strategy and the revealed preferences, derive the payment rule $\mathcal{P}\left(X^{\mathcal{N}^{*}}(\mathcal{B})\right) \in \mathbb{R}^{N}$, whose entries $\mathcal{P}_{j}\left(X^{\mathcal{N}^{*}}(\mathcal{B})\right)$ represent the revenue (if $\mathcal{P}_{j}\left(X^{\mathcal{N}^{*}}(\mathcal{B})\right) \geq 0$ ) or payment (if $\mathcal{P}_{j}\left(X^{\mathcal{N}^{*}}(\mathcal{B})\right) \leq 0$ ) of each prosumer $j \in \mathcal{N}$.

This mechanism is similar to a so-called indirect mechanism described in Nisan et al. (2007) and Khazaei \& Zhao (2019).

The proposed consumer-centric market mechanism must provide institutions and trading mechanisms that coordinate the interactions between the prosumers in the community towards a social choice. Firstly, the structure of the bids must adequately represent the physical and economic 
characteristics $\mathcal{B}$ of non-conventional flexibility assets, such as prosumers at the interface of heat and electricity systems. These prosumers may derive flexibility from fuel-shift technologies, such as heat-pumps and electric boilers (Meibom et al., 2007), as well as from exploiting the thermal dynamics of their buildings and shifting thermostatically controlled loads (Lesage-Landry \& Taylor, 2017; Dominković et al., 2018). As outlined in Liu et al. (2015), the classic bid formats may fail to represent these complex techno-economic characteristics. Secondly, the outcomes of the proposed mechanism $\left(X^{\mathcal{N}^{*}}(\mathcal{B}), \mathcal{P}\left(X^{\mathcal{N}^{*}}(\mathcal{B})\right)\right)$ must guarantee certain desirable properties, introduced in Section 2.2 in order to incentivize the prosumers to cooperate towards a social choice.

The proposed structure of the bids $\mathcal{B}$, the energy management problem of the community manager, and the form of the payment rule $\mathcal{P}\left(X^{\mathcal{N}^{*}}(\mathcal{B})\right)$ are discussed in the remainder of this section. Once this mechanism has been defined, its properties will be analyzed using the gametheoretic tools described in Section 2.3.

\subsection{Model of individual agents with private information: bid formats}

Individual agents participating in the proposed mechanism are prosumers at the interface between heat and electricity systems. As illustrated in Figure 1, for each prosumer $j \in \mathcal{N}$, we can distinguish four main types of flexible loads over all hours $t \in \mathcal{T}$ of the day, namely space heating, domestic hot water consumption, electricity load of heat pump, and other electrical appliances. In order to model the flexibility of space heating loads, the temperature dynamics of the buildings are represented through a set of linear equations detailed in Appendix B. The utility derived from space heating is defined with respect to a vector of temperature comfort level $T_{j}^{\text {set }} \in \mathbb{R}^{T}$ and flexibility range $\bar{u}_{j}^{\text {up }} \in \mathbb{R}_{+}^{T}, \bar{u}_{j}^{\text {down }} \in \mathbb{R}_{+}^{T}$ preferences. It can be supplied by district heating, electricity (via heat pumps), or heat storage tanks.

At each hour of the following day $t \in \mathcal{T}$, these loads may be supplied by heat and electricity exchanged with the grids or within the community, as well as by production from thermal generation, renewable energy, heat pumps, or storage units, as illustrated in Figure 1. These represent the space of decision variables of each prosumer in the community. Furthermore, a set of state variables, including the state of charge of heat and electricity storage units, the building's fabric temperatures, and upward and downward deviations from the indoor temperature comfort level at each hour of the following day $t \in \mathcal{T}$, represents the state of the prosumer and may influence its utility. This set of variables for all prosumers in the community $j \in \mathcal{N}$ and over all hours $t \in \mathcal{T}$ of the day is denoted by the matrix $X=\left[X_{1}, \ldots, X_{N}\right] \in \mathbb{R}^{K_{2} \times N}$, where each column $X_{j} \in \mathbb{R}^{K_{2}}$ represents the decision and state variables of a prosumer $j \in \mathcal{N}$ over all hours $t \in \mathcal{T}$ of the day.

Each prosumer $j \in \mathcal{N}$ holds private information that represents its load utilities and production costs, for all hours $t \in \mathcal{T}$ of the day, and the thermal characteristics of its building and the assets it owns. This set of true private preferences is denoted by $\tilde{\mathcal{B}}_{j} \in \mathbb{R}^{K_{1}}$. Prosumers are rational and selfinterested, i.e., seeking to maximize their individual utilities, through their interactions with the rest of the community within the institutions of the proposed mechanism. For that, each prosumer submits bids to the community manager, denoted by the vector $\mathcal{B}_{j} \in \mathbb{R}^{K_{1}}$. The structure of the bids $\mathcal{B}_{j} \in \mathbb{R}^{K_{1}}$ impacts how the prosumers can represent and communicate their preferences. In order to embed the complex techno-economic characteristics of the prosumers at the interface between heat and electricity systems, in this community-based market mechanism, the proposed bid format is represented by a set of matrices. Firstly, a cost matrix $Q^{\mathrm{c}}=\left[Q_{1}^{\mathrm{c}}, \ldots, Q_{N}^{\mathrm{c}}\right] \in \mathbb{R}^{K_{4} \times N}$, whose columns $Q_{j}^{\mathrm{c}} \in \mathbb{R}^{K_{4}}$ represent the vectors of variable load utilities and production costs of a prosumer $j \in \mathcal{N}$ over all hours $t \in \mathcal{T}$ of the day. Additionally, the technical characteristics of the prosumers are 
represented by the set of matrices $Q^{(0)} \in \mathbb{R}^{K_{3} \times N}$ and $Q^{(1)} \in \mathbb{R}^{K_{2} \times N}$, whose columns $Q_{j}^{(0)} \in \mathbb{R}^{K_{3}}$ and $Q_{j}^{(1)} \in \mathbb{R}^{K_{2}}$ represent the vectors of technical parameters of a prosumer over all hours of the day. For notational simplicity, dimensions $K_{3}$, and $K_{4}$ represent the lengths of the vectors of preferences of the prosumers over all hours of the day, such that $K_{1}=K_{2}+K_{3}+K_{4}$. These matrices can be derived from the compact and detailed expressions of the energy management problem, which are introduced in Appendix B. Contrary to the basic price-quantity bids, this generalized bid format allows the prosumers to model their cross-carrier flexibility and the synergies between different types of loads by a combination of linear constraints. These technical and economic parameters are detailed in Appendix B.

\subsection{Energy management strategy of the community manager}

The community manager chooses the optimal energy dispatch $X^{\mathcal{N}^{*}}(\mathcal{B})$ that maximizes the social welfare of the community with respect to the submitted bids $\mathcal{B}$. The linear objective function of the energy management problem is denoted by $\mathcal{H}\left(X, Q^{\mathrm{c}}\right)$. This objective function is separable, with respect to each prosumer $j \in \mathcal{N}$. Additionally, we can differentiate the components $\mathcal{F}_{j}\left(X_{j}, Q_{j}^{\mathrm{c}}\right)$ of the objective function which depend on the bids $Q_{j}^{\mathrm{c}}$ of the prosumers, representing the load utilities minus production costs, and the additional revenue $\mathcal{G}_{j}\left(X_{j}\right)$ from exchanges with the grids. The expression of these functions are detailed in Appendix B. As a result, the social welfare of the community can be expressed as

$$
\mathcal{H}\left(X, Q^{\mathrm{c}}\right)=\sum_{j \in \mathcal{N}} \mathcal{H}_{j}\left(X_{j}, Q_{j}^{\mathrm{c}}\right)=\sum_{j \in \mathcal{N}}\left(\mathcal{F}_{j}\left(X_{j}, Q_{j}^{\mathrm{c}}\right)+\mathcal{G}_{j}\left(X_{j}\right)\right) .
$$

The community manager ensures that heat and electricity consumption and production are balanced, by enforcing the following constraints:

$$
\begin{aligned}
& f^{\mathrm{E}} \mathbf{1}_{N}=\mathbf{0}_{T} \\
& f^{\mathrm{H}} \mathbf{1}_{N}=\mathbf{0}_{T},
\end{aligned}
$$

where the matrices $f^{\mathrm{E}} \in \mathbb{R}^{T \times N}$ and $f^{\mathrm{H}} \in \mathbb{R}^{T \times N}$ represent the electricity and heat exchanges, respectively, within the community. The feasible space of any coalition of prosumers $\mathcal{S} \in 2^{\mathcal{N}}$ with respect to the submitted bids $\mathcal{B}$ is represented by a set of linear equations, i.e.,

$$
\Omega_{\mathcal{S}}(\mathcal{B})=\left\{X \in \mathbb{R}^{K_{2} \times N} \mid(4 \mathrm{a})-(4 \mathrm{~b}), Q^{(2)}\left(X \circ Q^{(1)}\right) \leq Q^{(0)^{\mathcal{S}}}\right\},
$$

where the operator o represents the Hadamard product, and the matrix $Q^{(2)} \in \mathbb{R}^{K_{3} \times K_{2}}$ represents the shape of the operating constraints defined by the community manager. Additionally, the matrices $Q^{(0)} \in \mathbb{R}^{K_{3} \times N}$ and $Q^{(1)} \in \mathbb{R}^{K_{2} \times N}$ represent the bids of the prosumers. The matrix $Q^{(0)^{\mathcal{S}}}$ is indexed by the coalition $\mathcal{S}$, which ensures that the column $Q_{j}^{(0)^{\mathcal{S}}}=\mathbf{0}_{K_{2}}$ for any prosumer $j \notin \mathcal{S}$. Operations on matrices indexed by coalitions are defined in Appendix A. In particular, $Q^{(0)^{\mathcal{S}}}=\left[Q_{1}^{(0)} \delta_{1}^{\mathcal{S}}, \ldots, Q_{N}^{(0)} \delta_{N}^{\mathcal{S}}\right]$, where the Dirac delta function is defined as

$$
\delta^{\mathcal{S}}: k \in \mathcal{N} \mapsto \delta_{k}^{\mathcal{S}}=\left\{\begin{array}{l}
1 \text { if } k \in \mathcal{S} \\
0 \text { otherwise }
\end{array} .\right.
$$

Note that, this feasible set is non-empty, because $\mathbf{0}_{K_{2} \times T}$ is always feasible, and compact, because it includes upper and lower bounds on all variables. A feasible solution with respect to the revealed 
preferences $\mathcal{B}$ is denoted by $X^{\mathcal{S}}(\mathcal{B}) \in \Omega_{\mathcal{S}}(\mathcal{B})$. In particular, this definition of the feasible set of a coalition guarantees that a feasible solution $X_{j}^{\mathcal{S}}(\mathcal{B})$ for any prosumer $j \notin \mathcal{S}$ is fixed to zero.

The energy management problem of any coalition $\mathcal{S} \in 2^{\mathcal{N}}$ with respect to these revealed preferences $\mathcal{B}$ can be expressed as the following linear program:

$$
\max _{X \in \Omega_{\mathcal{S}}(\mathcal{B})} \mathcal{H}\left(X, Q^{\mathrm{c}}\right) .
$$

This optimization problem has a linear objective function and is defined on a convex, non-empty, and compact feasible set, hence it attains a global optimum $\theta^{\mathcal{S}}(\mathcal{B})=\mathcal{H}\left(X^{\mathcal{S}^{*}}(\mathcal{B}), Q^{\mathrm{c}}\right)$ at an optimal solution, i.e.,

$$
X^{\mathcal{S}^{*}}(\mathcal{B}) \in \arg \max _{X \in \Omega_{\mathcal{S}}(\mathcal{B})} \mathcal{H}\left(X, Q^{\mathrm{c}}\right) .
$$

Note that the optimal solution $X^{\mathcal{S}^{*}}(\mathcal{B})$ of $(7)$ and the optimal value $\theta^{\mathcal{S}}(\mathcal{B})$ are dependent on the bids of the prosumers in the coalition. In particular, the individual energy management problem is a special case of (7) for the coalition $\mathcal{S}=\{j\}$. The individual problem represents a base-case that we use for comparison purposes against the coalitional energy management problem. The detailed formulation of this mathematical problem is presented in Appendix B.

As previously mentioned, in the proposed mechanism the value $v^{\mathcal{S}}(\mathcal{B})$ of a coalition $\mathcal{S} \in 2^{\mathcal{N}}$ is defined as the increase in social welfare between the individual problems of all prosumers $j \in \mathcal{S}$ and the coalitional problem, with respect to the revealed preferences $\mathcal{B}$, such that

$$
v^{\mathcal{S}}(\mathcal{B})=\theta^{\mathcal{S}}(\mathcal{B})-\sum_{j \in \mathcal{S}} \theta^{\{j\}}(\mathcal{B})
$$

The revealed utility of a prosumer $j \in \mathcal{N}$ in this mechanism, which is defined as the utility with respect to its revealed preferences $\mathcal{B}_{j}$ evaluated at the outcomes $X^{\mathcal{N}^{*}}(\mathcal{B})$, can be formulated as

$$
u_{j}\left(X^{\mathcal{N}^{*}}(\mathcal{B}), \mathcal{B}\right)=\mathcal{P}_{j}\left(X^{\mathcal{N}^{*}}(\mathcal{B})\right)+\mathcal{F}_{j}\left(X_{j}^{\mathcal{N}^{*}}(\mathcal{B}), Q_{j}^{\mathrm{c}}\right)=\pi_{j}\left(X^{\mathcal{N}^{*}}(\mathcal{B})\right)+\theta^{\{j\}}(\mathcal{B}), \quad \forall j \in \mathcal{N} .
$$

By opposition, the true utility $u_{j}\left(X^{\mathcal{N}^{*}}(\mathcal{B}),\left(\tilde{\mathcal{B}}_{j}, \mathcal{B}_{-j}\right)\right)$ of a prosumer is defined as the utility with respect to its true preferences $\tilde{\mathcal{B}}_{j}$ evaluated at the outcomes $X^{\mathcal{N}^{*}}(\mathcal{B})$. This highlights the direct relationship between the payment rule of a mechanism and the allocation mechanism of the coalitional game form associated with this mechanism. As a result, the outcomes of the proposed mechanism with respect to the revealed preferences $\mathcal{B}$ can equivalently be given by the pair of optimal dispatch and payment rule $\left(X^{\mathcal{N}^{*}}(\mathcal{B}), \mathcal{P}\left(X^{\mathcal{N}^{*}}(\mathcal{B})\right)\right)$, or optimal dispatch and allocation $\operatorname{mechanism}\left(X^{\mathcal{N}^{*}}(\mathcal{B}), \pi\left(X^{\mathcal{N}^{*}}(\mathcal{B})\right)\right)$.

\subsection{Payment rule and allocation mechanism}

This section introduces different types of payment rules and allocation mechanisms that are commonly used in mechanism design. The properties of the proposed community-based market mechanism associated with these payments and allocation mechanisms will be studied in Section 4 . 


\subsubsection{Uniform pricing}

In the energy management problem (7), prosumers exchange heat and electricity within the community. Thus, by analogy with wholesale electricity markets, a common payment rule that can be used for exchanges within the community is based on uniform pricing. The revenues of each prosumer from exchanges within the community and with the grids are defined as

$$
\begin{aligned}
\mathcal{P}_{j}^{\lambda}\left(X_{j}^{\mathcal{N}^{*}}(\mathcal{B}), \lambda^{\mathcal{N}^{*}}(\mathcal{B})\right)= & \left(\lambda^{\mathrm{E}^{\mathcal{N}^{*}}}(\mathcal{B})\right)^{\top} f_{j}^{\mathrm{E}^{\mathcal{N}^{*}}}(\mathcal{B})+\left(\lambda^{\mathrm{H}^{\mathcal{N}^{*}}}(\mathcal{B})\right)^{\top} f_{j}^{\mathrm{H}^{\mathcal{N}^{*}}} \\
& +\mathcal{G}_{j}\left(X_{j}^{\mathcal{N}^{*}}(\mathcal{B})\right), \quad \forall j \in \mathcal{N}
\end{aligned}
$$

where $f^{E^{\mathcal{N}^{*}}}(\mathcal{B})$ and $f^{E^{\mathcal{N}^{*}}}(\mathcal{B})$ represent the value of electricity and heat exchanges within the community, at an optimal solution $X^{\mathcal{N}^{*}}(\mathcal{B})$ of $(7)$. Additionally, the uniform prices $\lambda^{E^{\mathcal{N}^{*}}}(\mathcal{B})$ and $\lambda^{H^{\mathcal{N}^{*}}}(\mathcal{B})$ represent the optimal value of the dual variables of $(7)$ associated with the balance equations (4a) and (4b) at the optimal solution $X^{\mathcal{N}^{*}}(\mathcal{B})$. Note that the value of both primal and dual variables at optimality are dependent on the submitted bids $\mathcal{B}$. For notational simplicity, we introduce the vector $\lambda^{\mathcal{N}^{*}}(\mathcal{B})=\left[\lambda^{\mathrm{E}^{\mathcal{N}^{*}}}(\mathcal{B}), \lambda^{\mathrm{H}^{\mathcal{N}^{*}}}(\mathcal{B})\right] \in \mathbb{R}^{2 T}$.

\subsubsection{Vickrey-Clarke-Groves (VCG)}

Another payment rule that has recently received increasing interest in the literature of electricity markets is the VCG payment rule with the Clarke pivot rule (Vickrey, 1961; Clarke, 1971; Groves, 1973; Green \& Laffont, 1979). The revenue of each prosumer with this payment rule is defined as

$$
\mathcal{P}_{j}^{\mathrm{VCG}}\left(X^{\mathcal{N}^{*}}(\mathcal{B})\right)=-\mathcal{F}_{j}\left(X_{j}^{\mathcal{N}^{*}}(\mathcal{B}), Q_{j}^{\mathrm{c}}\right)+\left(\theta^{\mathcal{N} \backslash\{j\}}(\mathcal{B})-\theta^{\mathcal{N}}(\mathcal{B})\right), \quad j \in \mathcal{N} .
$$

This payment rule represents the marginal contribution of each prosumer to the social welfare of the community. A series of recent studies has proposed VCG mechanisms applied to electricity markets, such as Karaca et al. (2019); Karaca \& Kamgarpour (2017); Sessa et al. (2017); Xu \& Low (2017); Exizidis et al. (2019).

\subsubsection{Shapley value}

An allocation mechanism commonly used in cooperative game theory is the Shapley value, where each prosumer receives an allocation as

$$
\pi_{j}^{\mathrm{s}}\left(X^{\mathcal{N}^{*}}(\mathcal{B})\right)=\sum_{\mathcal{S} \in 2^{\mathcal{N}}} \frac{(|\mathcal{S}|-1) !(N-|\mathcal{S}|) !}{N !}\left(v^{\mathcal{S}}(\mathcal{B})-v^{\mathcal{S} \backslash\{j\}}(\mathcal{B})\right) \delta_{j}^{\mathcal{S}}, \quad \forall j \in \mathcal{N} .
$$

Note that the sum of the coefficients in the left-hand side of (13) is equal to one, i.e.,

$$
\sum_{S \in 2^{\mathcal{N}}} \frac{(|\mathcal{S}|-1) !(N-|\mathcal{S}|) !}{N !} \delta_{j}^{\mathcal{S}}=1
$$

Therefore, this allocation mechanism associates to each prosumer $j \in \mathcal{N}$ its average marginal contribution to the values of all coalitions $\mathcal{S} \in 2^{\mathcal{N}}$. It is worth mentioning that the payment rule based on Shapley value requires computing the outcomes of the mechanism over all coalitions in the community, which is computationally intensive. 


\subsubsection{Nucleolus}

Finally, a well-known allocation mechanism in cooperative game theory is the nucleolus allocation $\pi^{\mathrm{n}}\left(X^{\mathcal{N}^{*}}(\mathcal{B})\right) \in \mathbb{R}^{N}$, which is defined as the allocation that minimizes the dissatisfaction of the prosumers with respect to the revealed information $\mathcal{B}$. In other words, the nucleolus is defined as the lexicographical maximum of the vector of excesses $\epsilon($.$) over all allocations, i.e.,$

$$
\epsilon_{\mathcal{S}}\left(\pi^{\mathrm{n}}\left(X^{\mathcal{N}^{*}}(\mathcal{B})\right)\right) \leq \epsilon_{\mathcal{S}}(\tilde{\pi}(\mathcal{B})), \quad \forall \tilde{\pi}(\mathcal{B}) \in \mathcal{I}(v(\mathcal{B})), \quad \forall \mathcal{S} \in 2^{\mathcal{N}} \backslash\{\mathcal{N}, \emptyset\}
$$

The nucleolus allocation always exists and is unique (Schmeidler, 1969). However, its computation requires solving a sequence of $\mathcal{O}\left(2^{N}\right)$ linear programs, which is computationally intensive. In practice, we compute the nucleolus of the cooperative game $(\mathcal{N}, v(\mathcal{B}))$ defined in $(9)$ by following steps (Guajardo \& Jörnsten, 2015; Han et al., 2018):

1. We generate a complete list of the values $v^{\mathcal{S}}(\mathcal{B})$ by solving the energy management problem (7) for all coalitions $\mathcal{S} \in 2^{\mathcal{N}}$.

2. We solve the following linear problem $\left(L P_{1}\right)$, which determines the maximum excess $\epsilon_{1}$ among all potential coalitions:

$$
\begin{array}{ll}
\epsilon_{1}=\min _{\epsilon, \pi \in \mathbb{R}^{N}} \epsilon \\
\text { s.t. } \quad \sum_{j=1}^{N} \pi_{j}=v^{\mathcal{N}}(\mathcal{B}) \\
& \pi_{j} \geq 0, \quad \forall j \in\{1, \ldots, N\} \\
& v^{\mathcal{S}}(\mathcal{B})-\sum_{j \in \mathcal{S}} \pi_{j} \leq \epsilon, \quad \forall \mathcal{S} \in 2^{\mathcal{N}} \backslash\{\mathcal{N}, \emptyset\},
\end{array}
$$

where (16b) enforces the efficient allocation property, and (16d) ensures that the excess of any coalition is lower than or equal to the maximum excess $\epsilon_{1} . L P_{1}$ gives us the set of coalitions $\Psi_{1}$ such that $(16 \mathrm{~d})$ is binding. However, the nucleolus allocation has not yet been computed.

3. At any iteration $l>1$, we solve a linear program $\left(L P_{l}\right)$ which determines the maximum excess $\epsilon_{l}$ over all coalitions that are not binding in the previous iterations $k<l$ :

$$
\begin{array}{ll}
\epsilon_{l}=\min _{\epsilon, \pi \in \mathbb{R}^{N}} & \epsilon \\
\text { s.t. } & (16 \mathrm{~b})-(16 \mathrm{c}) \\
& v^{\mathcal{S}}(\mathcal{B})-\sum_{j \in \mathcal{S}} \pi_{j} \leq \epsilon_{k}, \quad \forall \mathcal{S} \in \Psi_{k}, \forall k \in\{1, \ldots, l-1\} \\
& v^{\mathcal{S}}(\mathcal{B})-\sum_{j \in \mathcal{S}} \pi_{j} \leq \epsilon, \quad \forall \mathcal{S} \in 2^{\mathcal{N}} \backslash\left\{\mathcal{N}, \emptyset, \bigcup_{k=1}^{l-1} \Psi_{k}\right\},
\end{array}
$$

where $(17 \mathrm{c})$ imposes that the excesses of all coalitions $\mathcal{S} \in \Psi_{k}$ that are binding at iteration $k<l$ are lower than or equal to the maximum excess $\epsilon_{k}$, which is defined as the optimal value of $\left(L P_{k}\right)$ at iteration $k<l$. Additionally, (17d) enforces that all excesses of the coalitions that are not binding in the previous iterations are lower than or equal to $\epsilon_{l} . L P_{l}$ gives us the set of coalitions $\Psi_{l}$ such that (17d) is binding.

4. When a unique optimal solution is found, the solution $\pi^{\mathrm{n}}\left(X^{\mathcal{N}^{*}}(\mathcal{B})\right)$ is the nucleolus allocation. 


\section{Properties of the proposed community-based market mechanism and payment rules}

This section analyzes the properties of the proposed community-based market mechanism along with different payment rules and allocation mechanisms. We first demonstrate the existence of coreselecting mechanisms for the proposed community-based market. In addition, we show that such a mechanism provides a trade-off between efficiency, incentive compatibility, and stabilility. We then discuss which specific payment rules and allocation mechanisms can achieve these desirable properties in the proposed community-based market mechanism. The proofs of these propositions are provided in Appendix C. For the sake of self-containment, other well-known properties are also discussed.

\subsection{Existence of core-selecting mechanisms}

It results directly from the definition of the value function in $(9)$ that $v^{\{i\}}(\mathcal{B})=v^{\emptyset}(\mathcal{B})=$ $0, \forall j \in \mathcal{N}$. In order to define the pair $(\mathcal{N}, v(\mathcal{B}))$ as a coalitional game form, the value function must be super-additive.

Proposition 1. The value function $v^{\mathcal{S}}(\mathcal{B})$ defined in $(9)$ is super-additive, and the pair $(\mathcal{N}, v(\mathcal{B}))$ defines a coalitional game form associated with the proposed community-based market mechanism.

The proof of Proposition 1 is provided in Appendix C. This property guarantees that the prosumers can benefit by forming the grand-coalition $\mathcal{N}$.

Despite this, it is common for practical cooperative games to have an empty core. The notion of balanced game is essential in cooperative game theory, as a cooperative game has a non-empty core if and only if it is balanced (Scarf, 1967).

Definition 6 (Balanced map). A map $\alpha: 2^{\mathcal{N}} \rightarrow[0 ; 1]$ is balanced if $\sum_{\mathcal{S} \in 2^{\mathcal{N}}} \alpha(\mathcal{S}) \delta_{j}^{\mathcal{S}}=1, \forall j \in$ $\{1, \ldots, N\}$.

Definition 7 (Balanced game). A cooperative game $(\mathcal{N}, v(\mathcal{B}))$ is balanced if for any balanced map $\alpha$,

$$
\sum_{\mathcal{S} \in 2^{\mathcal{N}}} \alpha(\mathcal{S}) v^{\mathcal{S}} \leq v^{\mathcal{N}}
$$

An interesting property of the proposed community-based market mechanism is that, for any revealed preferences $\mathcal{B}$, the cooperative game defined by the pair $(\mathcal{N}, v(\mathcal{B}))$ is balanced, and thus has a non-empty revealed core.

Proposition 2. For any preferences $\mathcal{B}$, the cooperative game defined by the pair $(\mathcal{N}, v(\mathcal{B}))$ is balanced.

The proof of Proposition 2 is provided in Appendix C. As a result of this proposition, there exists a core-selecting mechanism $\left(X^{\mathcal{N}^{*}}(\mathcal{B}), \pi\left(X^{\mathcal{N}^{*}}(\mathcal{B})\right)\right)$ that redistributes the value of the energy community among the agents in a fair way, with respect to the revealed preferences $\mathcal{B}$. This property is fundamental in mechanism design, as it shows that there exists an adequate community-based mechanism that incentivizes the prosumers in the community to cooperate towards a social choice.

Furthermore, the outcomes $X^{\mathcal{N}^{*}}(\mathcal{B})$ of the energy management problem (7) maximize the social welfare of the community with respect to the revealed preferences $\mathcal{B}$. In order to guarantee that the true social welfare of the energy community is maximized, an allocation mechanism should provide incentives for agents to bid truthfully. Hence, the issue of modeling the behavior of the producers is central. We demonstrate an important linkage between the stability and incentive compatibility properties of the proposed cooperative game. 
Proposition 3 (incentive optimality). Any core-selecting mechanism $\left(X^{\mathcal{N}^{*}}(\mathcal{B}), \pi\left(X^{\mathcal{N}^{*}}(\mathcal{B})\right)\right)$ of the cooperative game $(\mathcal{N}, v(\mathcal{B}))$ defined in $(9)$ is Pareto efficient for the agents, and therefore, guarantees incentive optimality.

This property arises from the structure of the energy procurement problem and the expression of the efficient allocation property in (1). This property is essential, as it ensures that, an adequately designed community-based mechanism selects an allocation that is Pareto efficient for the agents, i.e. that maximizes the incentives for the agents to bid truthfully (Day \& Milgrom, 2008). In practice, the main challenge in designing such a mechanism relies in the choice of the payment allocation mechanism.

\subsection{Properties of payment rules and allocation mechanisms}

\subsubsection{Stability}

The stability property is fundamental in the design of a community-based market, as in ensures that the prosumers are satisfied with the outcomes of the mechanism, and that no coalition is willing to split from the community. Without this property, an energy community would not be able to form. Among the proposed payment and allocation mechanisms, solely the nucleolus is known to be in the revealed core of any cooperative game when this set is non-empty, i.e., the game is balanced (Peters, 2015). Therefore, the proposed community-based market along with a nucleolus allocation mechanism defines a core-selecting mechanism.

Although the Shapley value is, by construction, budget-balanced, there is no guarantee that it is in the revealed core of a cooperative game, even when this set is non-empty. In the case of the proposed community-based market mechanism, the case study in Section 5 shows that the Shapley value is not in the core of this game.

Furthermore, the VCG payment mechanism is not guaranteed to be budget-balanced or respect group rationality in the general case (Cavallo, 2006; Ausubel \& Milgrom, 2006). Therefore, the community manager may incur financial losses, and prosumers are not guaranteed to be satisfied with the outcomes of the mechanism. In fact, Karaca et al. (2019) show that the VCG mechanism is in the core of a game if and only if the objective value $\theta^{\mathcal{N}}(\mathcal{B})$ is submodular, i.e., has decreasing differences, which does not apply to the proposed community-based market mechanism. The shortcomings of the VCG mechanism are explained in detail in Ausubel \& Milgrom (2006); Sessa et al. (2017).

Finally, the uniform pricing payment mechanism is not in the revealed core of this cooperative game in the general case. Indeed, in the absence of network constraints or by redistributing congestion rents to transmission owners, the balance equations in (4a) and (4b) guarantee budget balance (Schweppe et al., 1988), but group rationality is not guaranteed. Yet, we demonstrate in Appendix $\mathrm{C}$ that under the assumption of perfect competition this allocation mechanism is in the revealed core of the cooperative game.

Proposition 4. Under the assumption of perfect competition, the community-based mechanism (7) with the payment rule $\mathcal{P}^{\lambda}$ based on uniform pricing is a core-selecting mechanism.

Note that the assumption of perfect competition is seldom verified in practice (Wolfram, 1997; Joskow \& Kahn, 2001), and may not be realistic for energy communities with a limited number of agents (Hurwicz, 1972). 


\subsubsection{Incentive compatibility}

Incentive compatibility is essential in mechanism design, as it ensure that the participants reveal their true preferences, and that the outcomes of this mechanism achieve a social choice with respect to these revealed preferences. In particular, in the case of small-scale community-based mechanisms, each prosumer may hold significant market power. Therefore, deviations from truthful bidding of any participant may result in significant changes in the social welfare of the community. Among the proposed payment and allocation mechanisms, solely the VCG mechanism is known to be dominant-strategy incentive-compatible (Hobbs et al., 2000; Silva et al., 2001; Zou et al., 2015; Xu \& Low, 2017; Sessa et al., 2017; Karaca \& Kamgarpour, 2017; Karaca et al., 2019).

Proposition 3 guarantees that any core-selecting mechanism is incentive-optimal. This property limits the benefits that prosumers can derive by deviating from truthful bidding. In addition, it is practically hard for a prosumer to form a strategy that can impact the excess over all coalitions, which is the basis of the nucleolus mechanism. Hence, the nucleolus allocation mechanism is defined in such a way that makes it practically difficult for agents to deviate from a truth-telling strategy.

In the general case with a finite number of agents, the proposed mechanism with uniform pricing is not incentive compatible. It is known that in order to maintain efficiency and incentive compatibility, perfect competition must be assumed (Wilson, 1977; Hobbs et al., 2004; Kazempour et al., 2018). Otherwise, the outcomes of the mechanism $X^{\mathcal{N}^{*}}(\mathcal{B})$ and $\mathcal{P}^{\lambda}\left(X_{j}^{\mathcal{N}^{*}}(\mathcal{B}), \lambda^{N^{*}}(\mathcal{B})\right)$ can be manipulated by the strategies $\mathcal{B}$ of individual agents. Additionally, Roberts \& Postlewaite (1976) show that incentive compatibility is achievable in the limit, as the number of agents grows to infinity. In such an ideal condition, this payment rule converges towards the VCG mechanism.

\subsubsection{Efficiency}

As the nucleolus allocation defines a core-selecting mechanism, it is a coalition-proof mechanism (Karaca et al., 2019). Furthermore, the proposed community-based market mechanism with a VCG payment is known to be efficient (Karaca et al., 2019). However, this mechanism with uniform pricing may not be efficient. Indeed, although the community manager seeks to maximize the social welfare of the community, Tang \& Jain (2013) show that a Nash equilibrium may not exist in the general case. However, under the assumption of perfect competition, and therefore incentive compatibility, this mechanism is indeed efficient.

\section{Illustrative case study: Impact and performance of the proposed mechanisms in a small community}

In this section the concepts and properties previously introduced are illustrated through a case study. This illustrative example shows in practice the value that can be achieved by a group of prosumers through cooperation, and briefly discusses how this value arises from the synergies between different types of loads. Furthermore, this case study highlights the impact of each payment rule and allocation mechanism on different types of prosumers, in terms of revenue and satisfaction. This case study setup and results are detailed in Appendix D.

\subsection{Individuals in the community}

We consider an energy community with twelve prosumers grouped into four categories. These prosumers wish to design a community-based market mechanism to exchange their local energy production, and coordinate their energy procurement from the grid. The two main challenges to 
motivate these prosumers to join the community are to demonstrate the value of cooperating and redistribute fairly the value created.

In this community, each category of prosumers has a different type of energy usage and flexibility, as gathered in Table 1. However, the prosumers in each category are considered to have identical loads, utilities, and flexible appliances. Therefore, each category is named after a stereotypical prosumer in it, namely Christos, Anna, Vladimir and Andrea. The Christos prosumers own an electric storage and rooftop solar panels, whose power generation is assumed to be perfectly known, and at a zero marginal cost. The Anna prosumers provide cross-carrier flexibility via a heat-pump, and own a heat storage. The Vladimir and Andrea prosumers have neither production units nor storage. However, The Andrea prosumers have flexible indoor temperatures whereas the Vladimir prosumers do not. In this case study, we model an asymmetric utility function for the deviations from the indoor temperature set-point, i.e., downward deviations are more penalized than upward deviations. Moreover, for simplicity, solely space heating loads and electricity consumption of heat pumps are assumed flexible. The electricity consumption of other electrical appliances and domestic hot water loads are considered inelastic and perfectly known for all prosumers.

Table 1: Flexible assets and loads of each category of prosumers in the community.

\begin{tabular}{lcccc} 
& Christos & Anna & Vladimir & Andrea \\
\hline Solar panel & Yes & No & No & No \\
Electricity storage & Yes & No & No & No \\
Heat storage & No & Yes & No & No \\
Heat pump & No & Yes & No & No \\
Electrical appliances & Inelastic & Inelastic & Inelastic & Inelastic \\
Domestic hot water & Inelastic & Inelastic & Inelastic & Inelastic \\
Space heating load & Elastic & Elastic & Inelastic & Elastic \\
\hline
\end{tabular}

In order to highlight the impact of the distribution of these stereotypical prosumers on the value of the community and the payment allocations to individual prosumers, we consider five scenarios, which correspond to different ratios of prosumers in each category, as summarized in Table 2.

Table 2: Number of prosumers in each category across five scenarios

Scenario 1 Scenario $2 \quad$ Scenario 3 Scenario 4 Scenario 5

\begin{tabular}{lccccc}
\hline Christos & 3 & 6 & 2 & 2 & 2 \\
Anna & 3 & 2 & 6 & 2 & 2 \\
Andrea & 3 & 2 & 2 & 6 & 2 \\
Vladimir & 3 & 2 & 2 & 2 & 6 \\
Total & 12 & 12 & 12 & 12 & 12 \\
\hline
\end{tabular}

These prosumers can exchange heat and electricity within the community, and with the grid. The import and export tariffs with the grid are modeled as hourly tariffs, of which the import price is systematically higher than the export price in order to account for grid tariffs. Heat and electricity export prices are proportional to the import prices, with a coefficient 0.9 , in order to reflect the effect of grid tariffs on end-users. For the sake of clarity and simplicity, we assume that the prosumers reveal their true preferences (though this is a dominant strategy under the VCG mechanism). Based on the true preferences of the prosumers, we solve the individual and 
community-based energy procurement problems for all hours of the following day. The results of this case study illustrate the impact of the community-based dispatch, different payment rules and allocation mechanisms on all four categories of prosumers.

Note that, due to the nature of the energy management problem and the payment allocation mechanisms considered, the energy dispatch and the payment allocation to each prosumer are solely dependent on the ratio of prosumers in each category. Therefore, this approach can directly be generalized to communities with a larger number of prosumers. In practice, communities with a large number of prosumers, e.g., 1,000 prosumers, can be modeled in such a way by designing appropriate contracts between the community manager and individual prosumers. Indeed, each prosumer can enter into a contract with the community manager, which groups the prosumers into multiple categories depending on their characteristics, such as load profiles, utilities of loads, flexible production units. In addition, this approach can be used to reduce the computational burden of computing the Shapley value and Nucleolus payment allocation mechanisms.

\subsection{Value of cooperation}

In this section, the impact of the community-based dispatch on the community as a whole and on individual prosumers is discussed. For each scenario, the community-based dispatch is compared to the individual dispatch, in which each prosumer optimizes its energy procurement from the grids on its own. The difference in social welfare of the community between these two cases represents the value of cooperating as defined in (9). As reported in Table 3, the social welfare of the community in each scenario is significantly increased compared to the individual case. This represents the value of cooperating for the community as a whole. This is achieved by exploiting the synergies between the prosumers and different types of loads. In particular, during the day, the community-based market mechanism is able to exploit the synergies between the cross-carrier flexibility offered by the heat pump belonging to Anna, and the excess electricity production from solar panels belonging to Christos. Additionally, during the night and evening, when electricity prices are low and heat demand is high, the community-based market mechanism is able to exploit the temperature flexibility of the prosumers, due to the heat pump belonging to Anna. These sources of flexibility and their synergies are under-utilized in the individual case. Additionally, the values of the community are heterogeneous across the five scenarios. The gain in social welfare is the highest in Scenario 3, i.e., for the highest ratio of Anna prosumers. These prosumers provide flexibility in both heat and electricity systems by utilizing their heat-pump. This cross-carrier flexibility allows the community to exploit the thermal flexibility of buildings, as well as the solar electricity production of the Christos prosumers. While the increase in social welfare in Scenario 2, i.e., for the highest ratio of Christos prosumers, is still significant, it is just around $50 \%$ of that in Scenario 3. The community is able to exploit the solar production of Christos prosumers to supply the electricity loads. However, these loads are less flexible that space heating loads. Therefore, the lack of cross-carrier flexibility provided by Anna prosumers prevents the community from taking more advantage of the solar production of Christos prosumers.

\subsection{Payment rules and allocation mechanisms}

This section discusses the impact of different allocation mechanisms on individual prosumers. Table 4 summarizes how different allocation mechanisms redistribute the increase in social welfare among the four categories of prosumers. Note that, due to the symmetry of the optimization problem and the payment allocation mechanisms, the payments to all prosumers in a given category are identical. As expected, the Shapley value, nucleolus, and uniform pricing mechanisms are 
Table 3: Social welfare in the individual and community-based cases, across Scenarios 1 to 5 (in $€$, and \%). The value of cooperation is calculated as the absolute difference in social welfare.

Social welfare Value of cooperation

\begin{tabular}{llcc}
\hline & Scenario 1 & $-285.3 €$ & - \\
Individual & Scenario 2 & $40.4 €$ & - \\
& Scenario 3 & $-117.5 €$ & - \\
& Scenario 4 & $-485.7 €$ & - \\
& Scenario 5 & $-578.6 €$ & - \\
\hline \multirow{5}{*}{ Community } & Scenario 1 & $-22.1 €$ & $+263.2 €$ \\
& Scenario 2 & $266.1 €$ & $+225.7 €$ \\
& Scenario 3 & $310.3 €$ & $+427.8 €$ \\
& Scenario 4 & $-307.7 €$ & $+178.1 €$ \\
& Scenario 5 & $-374.1 €$ & $+204.5 €$ \\
\hline
\end{tabular}

efficient allocations, i.e., they are budget-balanced. However, the VCG mechanism incurs losses for the community manager, as the total allocation attributed to the prosumers exceeds the value of the community. This is a significant shortcoming for this mechanism, and an ex-post redistribution is required (Cavallo, 2006). Furthermore, the four allocation mechanisms considered are individually rational in this example, i.e., the allocations to all prosumers are positive. This guarantees that the prosumers are willingly participating in the proposed mechanisms.

Table 4: Allocation of the value of the community attributed to each (individual) prosumer, and total allocations (in $€$ ), across different allocation mechanisms and scenarios.

\begin{tabular}{llccccc} 
& Christos & Anna & Vladimir & Andrea & Total \\
\hline Scenario 1 & Uniform pricing & 14.6 & 63.8 & 1.4 & 8.0 & 263.2 \\
& VCG & 16.1 & 63.8 & 1.3 & 8.0 & 267.7 \\
& Shapley & 16.3 & 57.8 & 4.3 & 9.3 & 263.2 \\
& Nucleolus & 15.1 & 63.6 & 1.2 & 7.8 & 263.2 \\
\hline Scenario 2 & Uniform pricing & 11.2 & 65.8 & 3.3 & 10.2 & 225.7 \\
& VCG & 11.2 & 79.6 & 3.5 & 10.4 & 254.1 \\
& Shapley & 8.7 & 69.8 & 6.1 & 10.7 & 225.7 \\
& Nucleolus & 11.2 & 65.8 & 3.3 & 10.2 & 225.7 \\
\hline Scenario 3 & Uniform pricing & 38.0 & 44.4 & 18.0 & 24.6 & 427.8 \\
& VCG & 47.2 & 62.4 & 27.3 & 34.2 & 591.9 \\
& Shapley & 41.7 & 37.9 & 26.3 & 32.1 & 427.8 \\
& Nucleolus & 45.7 & 35.7 & 27.3 & 33.9 & 427.8 \\
\hline Scenario 4 & Uniform pricing & 20.0 & 62.5 & 0.0 & 6.6 & 178.1 \\
& VCG & 20.0 & 62.5 & 0.0 & 6.6 & 178.1 \\
& Shapley & 17.1 & 59.2 & 2.1 & 6.5 & 178.1 \\
& Nucleolus & 19.8 & 62.5 & 0.0 & 6.7 & 178.1 \\
\hline Scenario 5 & Uniform pricing & 20.0 & 62.5 & 0.0 & 6.6 & 204.5 \\
& VCG & 20.0 & 65.4 & 0.0 & 6.6 & 210.4 \\
& Shapley & 17.1 & 63.9 & 2.1 & 6.4 & 204.5 \\
& Nucleolus & 19.4 & 62.9 & 0.0 & 6.6 & 204.5 \\
\hline
\end{tabular}


Furthermore, it can be observed in Table 4 that the allocations to each prosumer vary greatly across the scenarios. In particular, as previously explained, the value of the community in Scenario 3 is the largest among the five scenarios due to the cross-carrier flexibility of Anna producers. However, the allocations to Anna producers decrease in Scenario 3 compared to the other scenarios, across all four allocation mechanisms. This can be explained by the large number of Anna producers in this scenario, among which to share this value. In other words, the community is saturated with flexible sources, and therefore, the marginal contribution of each individual flexible prosumer is lower compared to the other scenarios.

In order to ensure fairness of the redistribution mechanism and the satisfaction of all prosumers, the chosen allocation mechanisms should be in the core of the cooperative game. Table 5 summarizes the maximum excess over all coalitions under different allocation mechanisms.

Table 5: Excess (in $€$ ), group rationality and stability of different payment rules and allocation mechanisms.

\begin{tabular}{|c|c|c|c|c|c|}
\hline & & Uniform pricing & VCG & Shapley & Nucleolus \\
\hline \multirow{3}{*}{ Scenario 1} & Max. excess & 0.0 & -1.3 & 12.9 & 0.0 \\
\hline & Group rationality & Yes & Yes & No & Yes \\
\hline & Stability & Yes & No & No & Yes \\
\hline \multirow{3}{*}{ Scenario 2} & Max. excess & 0.0 & -1.0 & 3.2 & 0.0 \\
\hline & Group rationality & Yes & Yes & No & Yes \\
\hline & Stability & Yes & No & No & Yes \\
\hline \multirow{3}{*}{ Scenario 3} & Max. excess & 0.0 & -27.3 & 1.3 & 0.0 \\
\hline & Group rationality & Yes & Yes & No & Yes \\
\hline & Stability & Yes & No & No & Yes \\
\hline \multirow{3}{*}{ Scenario 4} & Max. excess & 0.0 & -1.0 & 9.4 & 0.0 \\
\hline & Group rationality & Yes & Yes & No & Yes \\
\hline & Stability & Yes & No & No & Yes \\
\hline \multirow{3}{*}{ Scenario 5} & Max. excess & 0.0 & -3.9 & 3.7 & 0.0 \\
\hline & Group rationality & Yes & Yes & No & Yes \\
\hline & Stability & Yes & No & No & Yes \\
\hline
\end{tabular}

For each scenario of this numerical example, the Shapley value is not in the core of the cooperative game because all coalitions that contain Anna prosumers have a positive excess. In practice, these coalitions have an incentive to split from the energy community to increase their utility. As a result, this allocation mechanism is not suitable to incentivize prosumers to form an energy community and to cooperate towards a social choice.

Furthermore, despite guaranteeing group rationality, the VCG mechanism is not in the core of the cooperative game because it is not budget balanced. As a result, despite the interesting properties of this allocation mechanism, it is not feasible in practice. Indeed, the community manager would need to design an additional redistribution mechanism to ensure budget balance. However, these mechanisms have been proven to violate the incentive-compatibility property Karaca et al. (2019).

In the presented scenarios, both the nucleolus and uniform-pricing allocation mechanisms are in the core of the cooperative game. However, as previously discussed, the uniform-pricing mechanism cannot be guaranteed to be a core-selecting mechanism. Indeed, budget-balance cannot be guaranteed if networks or centrally-operated energy storages are accounted for. Additionally, in- 
centive compatibility cannot be reasonably assumed for a small number of prosumers. This makes this allocation mechanism impractical for small-scale energy communities.

Finally, these numerical results validate the theoretical properties the nucleolus allocation mechanism, and show that a community-based energy market with this allocation mechanism provides adequate incentives for prosumers to cooperate truthfully and maximize the social welfare of the community.

\section{Conclusion and future research directions}

The community-based market mechanisms developed in this work provides a promising framework to coordinate the energy management of prosumers in local multi-carrier energy markets. This work highlights the value of harnessing the synergies between different types of loads and prosumers. The theoretical and applied contributions presented in this paper show that it is beneficial for the community as a whole to cooperate. However, it is known that no mechanism can satisfy stability, dominant-strategy incentive compatibility, and efficiency in the general case (Roth, 1982). Therefore, the four payment rules and allocation mechanisms studied only achieve certain of these fundamental mechanism properties. In particular, the nucleolus allocation is the only allocation that can guarantee stability in the general case. Furthermore, we demonstrate that it provides an interesting trade-off between stability, incentive-optimality, and efficiency. However, its main limitation is its computational complexity.

This work opens up various directions for future research, directed at consumer-centric market mechanism designers, as well as wholesale market operators and regulators. Firstly, uncertainty on the states of the system and exogenous perturbations should be accounted for in the optimal energy management of the community. Various studies have proposed robust control approaches for communities of buildings in multi-carrier energy systems via linear decision rules (Rey et al., 2019; Darivianakis et al., 2017a,b). Such approaches model the coordinated response of prosumers to exogenous deviations both from the grids, e.g., price signals or reserve activation, and outside conditions, e.g., renewable energy production of the community and temperatures. This approach would allow a better coordination between day-ahead scheduling and real-time operation of the flexible assets of the community, and reveal financial opportunities for this operational flexibility. For that purpose, wholesale market mechanisms should evolve in order to integrate prosumers and other decentralized flexibility assets, such as micro CHPs, energy storage, across multiple energy systems. Novel trading mechanisms facilitating market access to these non-conventional sources of flexibility must be developed. In particular, a promising research direction consists of affine reserve policies, that model the coordinated response of flexible assets to deviations across multiple energy systems, time horizons and time scales (Warrington et al., 2013).

Furthermore, despite the theoretical guarantees on the proposed allocation mechanisms, the agents in this community may deviate from truthful bidding for strategic reasons, i.e., in order to increase their profits, or for privacy concerns. This raises the issue of equilibrium in games with asymmetric information. In particular, privacy concerns should be accounted for in the design of peer-to-peer and community-based market mechanisms. A widely used approach to preserve privacy is differential privacy, which protects sensitive information by adding systematic noise to the revealed preferences of the agents (Dwork \& Roth, 2014; Dwork, 2008). An interesting extension to this work would be to investigate differentially-private consumer-centric market mechanisms. From a theoretical perspective, a number of recent studies in mechanism design have focused on the issue of truthfulness and fairness of differentially-private mechanisms. In addition to the intrinsic 
benefit of preserving private information, McSherry \& Talwar (2007) show that differentially private mechanisms can reduce the effect of the strategic behavior of the agents on the outcomes of the mechanism, and thus limit their incentives to misreport. Additionally, Forges \& Minelli (2001) and Forges et al. (2001) study the relationship between incentive compatibility, stability, and efficiency of differentially private mechanisms. Furthermore, privacy guarantees in repeated games deteriorate, due to the accumulation of observations. Therefore, the learning capabilities and the strategic behavior of agents in dynamic games should be investigated (Angeletos et al., 2007).

An alternative research direction to preserve privacy in decentralized optimization problem and mechanism designs, is to allow prosumers to communicate endogenous uncertainty sets of their preferences to the community. Building on the work by Darivianakis et al. (2018) and Zhang et al. (2017) the properties of a consumer-centric market mechanism with adjustable uncertainty sets should be investigated. From a technical perspective, the convergence and the trade-off between optimality and privacy of differentially-private decentralized optimization algorithms should be studied (Huang et al., 2015; Nozari et al., 2018; Bellet et al., 2017; Vanhaesebrouck et al., 2017). These algorithms, based on Lagrangian relaxation and the alternating direction of multipliers methods, may facilitate decentralized decision-making over peer-to-peer networks, while preserving privacy over sensitive information. However, they may fail to converge to a global optimal solution.

Finally, in order to fully exploit the potential of these decentralized flexibility assets, consumercentric market mechanism designers, market operators and regulators need a better understanding of the motivations and behavior of prosumers and market participants. In particular, the field of behavioral economy and prospect theory (Kahneman \& Tversky, 2013) provides a promising research direction by challenging the assumption of perfect rationality of agents in markets (Arthur, 1994; Sargent et al., 1993; Simon, 1997; Gigerenzer \& Selten, 2002). In particular, such models have been applied to analyze the behavior of prosumers in energy markets (Stern, 1992; Sanstad \& Howarth, 1994; Jaffe \& Stavins, 1994; Wilson \& Dowlatabadi, 2007; Gyamfi et al., 2013). Thus, integrating the notion of bounded rationality in community-based market mechanism design, and analyzing the properties of these mechanisms under this assumption would be an interesting extension of the work presented (Ming \& Xie, 2014). Moreover, the effect of bounded rationality on wholesale markets and energy systems should be studied. Indeed, accounting for bounded rationality in the response of agents to demand response programs in energy policy and market design may help increase the cost-effectiveness of existing organizations (Allcott \& Mullainathan, 2010).

\section{Appendix A. Notations}

This appendix summarizes the main notations and conventions used in this study.

\section{Appendix A.1. Nomenclature}

Sets and indexes

$\mathcal{T}$ Set of $T$ time periods, typically the 24 hours of a day

$\mathcal{N}$ Grand-coalition of $N$ prosumers

$2^{\mathcal{N}}$ Set of all coalitions in the grand-coalition $\mathcal{N}$

$\mathcal{S}, \mathcal{V}$ Coalitions of prosumers in the grand-coalition $\mathcal{N}$ 
$\tilde{\mathcal{B}}$ True preferences of the prosumers in the community, in $\mathbb{R}^{K_{1} \times N}$

$\mathcal{B}$ Revealed preferences of prosumers in the community, in $\mathbb{R}^{K_{1} \times N}$

$\Omega_{\mathcal{S}}(\mathcal{B})$ Feasible region of the energy management problem of coalition $S$, given the bids $\mathcal{B}$ of prosumers

\section{Parameters}

$Q^{c}$ Matrix of revealed price preferences, in $\mathbb{R}^{K_{4} \times N}$

$Q^{(0)}$ Matrix of revealed technical parameters, in $\mathbb{R}^{K_{3} \times N}$

$Q^{(1)}$ Matrix of revealed technical parameters, in $\mathbb{R}^{K_{2} \times N}$

$Q^{(2)}$ Matrix representing the constraints of the energy management problem, in $\mathbb{R}^{K_{3} \times K_{2}}$

$c^{\mathrm{b}, \mathrm{H}}$ Vector of heat import prices, in $\mathbb{R}^{T}(€ / \mathrm{Wh})$

$c^{\mathrm{b}, \mathrm{E}}$ Vector of electricity import prices, in $\mathbb{R}^{T}(€ / \mathrm{Wh})$

$c^{\mathrm{s}, \mathrm{H}}$ Vector of heat export prices, in $\mathbb{R}^{T}(€ / \mathrm{Wh})$

$c^{\mathrm{s}, \mathrm{E}}$ Vector of electricity export prices, in $\mathbb{R}^{T}(€ / \mathrm{Wh})$

$c^{\mathrm{g}, \mathrm{H}}$ Matrix of heat production costs, in $\mathbb{R}^{T \times N}(€ / \mathrm{Wh})$

$c^{\mathrm{g}, \mathrm{E}}$ Matrix of electricity production costs, in $\mathbb{R}^{T \times N}(€ / \mathrm{Wh})$

$c^{\mathrm{E}}$ Matrix of elasticity parameters of electricity consumption, in $\mathbb{R}^{T \times N}(€ / \mathrm{Wh})$

$c^{\mathrm{HW}}$ Matrix of elasticity parameters of hot water consumption, in $\mathbb{R}^{T \times N}(€ / \mathrm{Wh})$

$c^{\mathrm{up}}$ Matrix of elasticity parameters of upward temperature deviations, in $\mathbb{R}^{T \times N}\left(€ /{ }^{\circ} \mathrm{C}\right)$

$c^{\text {down }}$ Matrix of elasticity parameters of upward temperature deviations, in $\mathbb{R}^{T \times N}\left(€ /{ }^{\circ} \mathrm{C}\right)$

COP Matrix of coefficient of performance of heat pumps, in $\mathbb{R}^{T \times N}$

$T^{\text {set }}$ Matrix of set points of indoor temperatures, in $\mathbb{R}^{T \times N}$

$e n^{\mathrm{E}, 0}$ Matrix of initial charging state of electricity storage, in $\mathbb{R}^{T \times N}$ (Wh)

$\rho^{\mathrm{ch}^{\mathrm{E}}}$ Matrix of charging efficiency coefficients of electricity storage, in $\mathbb{R}^{T \times N}$

$\rho^{\mathrm{dis}, \mathrm{E}}$ Matrix of discharging efficiency coefficients of electricity storage, in $\mathbb{R}^{T \times N}$

$e n^{\mathrm{H}, 0}$ Matrix of initial charging state of heat storage, in $\mathbb{R}^{T \times N}(\mathrm{Wh})$

$\rho^{\mathrm{ch}, \mathrm{H}}$ Matrix of charging efficiency coefficients of heat storage, in $\mathbb{R}^{T \times N}$

$\rho^{\mathrm{dis}, \mathrm{H}}$ Matrix of discharging efficiency coefficients of heat storage, in $\mathbb{R}^{T \times N}$ 
$\rho^{\mathrm{E}}$ Matrix of electricity efficiency coefficients of thermal units, in $\mathbb{R}^{T \times N}$

$\rho^{\mathrm{H}}$ Matrix of heat efficiency coefficients of thermal units, in $\mathbb{R}^{T \times N}$

$r$ Matrix of minimum heat to power ratios of thermal units, in $\mathbb{R}^{T \times N}$

$\bar{b}^{\mathrm{E}}$ Matrix of upper bounds for electricity import from the grid, in $\mathbb{R}^{T \times N}$

$\bar{s}^{\mathrm{E}}$ Matrix of upper bounds for electricity export to the grid, in $\mathbb{R}^{T \times N}$ (Wh)

$\bar{b}^{\mathrm{H}}$ Matrix of upper bounds for heat import from the grid, in $\mathbb{R}^{T \times N}$ (Wh)

$\bar{s}^{\mathrm{H}}$ Matrix of upper bounds for heat export to the grid, in $\mathbb{R}^{T \times N}$ (Wh)

$\bar{f}^{\mathrm{E}}$ Matrix of upper bounds for electricity exchange within the community, in $\mathbb{R}^{T \times N}$

$\bar{f}^{\mathrm{H}}$ Matrix of upper bounds for heat exchange within the community, in $\mathbb{R}^{T \times N}$ (Wh)

$\bar{\Phi}^{\mathrm{E}}$ Matrix of upper bounds for electricity production from thermal generation, in $\mathbb{R}^{T \times N}$

$\bar{\Phi}^{\mathrm{H}}$ Matrix of upper bounds for heat production from thermal generation, in $\mathbb{R}^{T \times N}(\mathrm{Wh})$

$\bar{\Phi}^{\mathrm{ENR}}$ Matrix of upper bounds for electricity production from renewable energy, in $\mathbb{R}^{T \times N}$ (Wh)

$\bar{F}$ Matrix of upper bounds for fuel consumption from thermal generation, in $\mathbb{R}^{T \times N}$ (Wh)

$\bar{L}^{\mathrm{HP}}$ Matrix of upper bounds for electricity consumption of heat-pump, in $\mathbb{R}^{T \times N}(\mathrm{Wh})$

$\overline{e n}^{\mathrm{E}}$ Matrix of upper bounds for state of charge of electricity storage, in $\mathbb{R}^{T \times N}$ (Wh)

$\overline{e n}^{\mathrm{H}}$ Matrix of upper bounds for state of charge of heat storage, in $\mathbb{R}^{T \times N}$ (Wh)

$\overline{c h}^{\mathrm{E}}$ Matrix of upper bounds for charging of electricity storage, in $\mathbb{R}^{T \times N}$ (Wh)

$\overline{c h}^{\mathrm{H}}$ Matrix of upper bounds for charging of heat storage, in $\mathbb{R}^{T \times N}$

$\overline{d i s}^{\mathrm{E}}$ Matrix of upper bounds for charging of electricity storage, in $\mathbb{R}^{T \times N}$ (Wh)

$\overline{\text { dis }}^{\mathrm{H}}$ Matrix of upper bounds for charging of heat storage, in $\mathbb{R}^{T \times N}$ (Wh)

$\bar{u}^{\text {up }}$ Matrix of upper bounds for upward indoor temperature deviations, in $\mathbb{R}^{T \times N}\left({ }^{\circ} \mathrm{C}\right)$

$\bar{u}^{\text {down }}$ Matrix of upper bounds for downward indoor temperature deviations, in $\mathbb{R}^{T \times N}\left({ }^{\circ} \mathrm{C}\right)$

$\bar{L}^{\mathrm{E}}$ Matrix of upper bounds for bounds of electrical appliances consumption, in $\mathbb{R}^{T \times N}(\mathrm{Wh})$

$\bar{L}^{\mathrm{HW}}$ Matrix of upper bounds for hot water consumption, in $\mathbb{R}^{T \times N}(\mathrm{Wh})$

$\bar{L}^{\mathrm{HP}}$ Matrix of upper bounds for electric consumption of heat pump, in $\mathbb{R}^{T \times N}$ (Wh)

$\bar{L}^{\mathrm{SH}}$ Matrix of upper bounds for space heating consumption, in $\mathbb{R}^{T \times N}$ (Wh) 
Primal variables of energy management problem

$X$ Matrix of primal variables of the energy management problem, in $\mathbb{R}^{K_{2} \times N}$

$b^{\mathrm{E}}$ Matrix of electricity import, in $\mathbb{R}^{T \times N}$ (Wh)

$s^{\mathrm{E}}$ Matrix of electricity export, in $\mathbb{R}^{T \times N}$ (Wh)

$b^{\mathrm{H}}$ Matrix of heat import, in $\mathbb{R}^{T \times N}(\mathrm{Wh})$

$s^{\mathrm{H}}$ Matrix of heat export, in $\mathbb{R}^{T \times N}(\mathrm{Wh})$

$f^{\mathrm{E}}$ Matrix of electricity exchange within the community, in $\mathbb{R}^{T \times N}(\mathrm{Wh})$

$f^{\mathrm{H}}$ Matrix of heat exchange within the community, in $\mathbb{R}^{T \times N}$ (Wh)

$L^{\mathrm{E}}$ Matrix of electrical appliances consumption, in $\mathbb{R}^{T \times N}(\mathrm{Wh})$

$L^{\mathrm{HW}}$ Matrix of hot water consumption, in $\mathbb{R}^{T \times N}(\mathrm{Wh})$

$L^{\mathrm{HP}}$ Matrix of electricity consumption for heat pump, in $\mathbb{R}^{T \times N}(\mathrm{Wh})$

$L^{\mathrm{SH}}$ Matrix of space heating consumption, in $\mathbb{R}^{T \times N}(\mathrm{Wh})$

$T^{\mathrm{a}}$ Matrix of indoor temperature, in $\mathbb{R}^{T \times N}\left({ }^{\circ} \mathrm{C}\right)$

$T^{\mathrm{s}}$ Matrix of buildings' fabric temperature, in $\mathbb{R}^{T \times N}\left({ }^{\circ} \mathrm{C}\right)$

$u^{\text {up }}$ Matrix of upward deviations from indoor temperature set points, in $\mathbb{R}^{T \times N}\left({ }^{\circ} \mathrm{C}\right)$

$u^{\text {down }}$ Matrix of downward deviations from indoor temperature set points, in $\mathbb{R}^{T \times N}\left({ }^{\circ} \mathrm{C}\right)$

$\Phi^{\mathrm{E}}$ Matrix of electricity production from thermal generation, in $\mathbb{R}^{T \times N}(\mathrm{Wh})$

$\Phi^{\mathrm{H}}$ Matrix of heat production from thermal generation, in $\mathbb{R}^{T \times N}(\mathrm{Wh})$

$\Phi^{\mathrm{ENR}}$ Matrix of electricity production from renewables, in $\mathbb{R}^{T \times N}$ (Wh)

$c h^{\mathrm{E}}$ Matrix of charging of electricity storage, in $\mathbb{R}^{T \times N}(\mathrm{Wh})$

$d i s^{\mathrm{E}}$ Matrix of discharging of electricity storage, in $\mathbb{R}^{T \times N}$ (Wh)

$e n^{\mathrm{E}}$ Matrix of state of charge of electricity storage, in $\mathbb{R}^{T \times N}$ (Wh)

$c h^{\mathrm{H}}$ Matrix of charging of heat storage, in $\mathbb{R}^{T \times N}(\mathrm{Wh})$

$d i s^{\mathrm{H}}$ Matrix of discharging of heat storage, in $\mathbb{R}^{T \times N}(\mathrm{Wh})$

$e n^{\mathrm{H}}$ Matrix of state of charge of heat storage, in $\mathbb{R}^{T \times N}(\mathrm{Wh})$

Functions 
$\mathcal{H}($.$) Objective function of the energy management problem, representing the social welfare of the$ community $(€)$

$\mathcal{F}_{j}($.$) Utility of loads minus production cost of each prosumer j \in \mathcal{N}(€)$

$\mathcal{G}_{j}($.$) Revenue of each prosumer j \in \mathcal{N}$ from exchanges with the grids (€)

$\mathcal{P}($.$) Vector of payment rules, whose entries \mathcal{P}_{j}($.$) are the revenues of each prosumer j \in \mathcal{N}(€)$

$v($.$) Value function defined over the set of coalitions 2^{\mathcal{N}}(€)$

$\pi($.$) Vector of allocations for a cooperative game (\mathcal{N}, v)$, whose entries $\pi_{j}($.$) are the allocation of$ each prosumer $j \in \mathcal{N}(€)$

$\epsilon($.$) Vector of excesses, whose entries \epsilon_{\mathcal{S}}($.$) are the excess of each coalition \mathcal{S} \in 2^{\mathcal{N}}(€)$

$u($.$) Vector of utilities, whose entries u_{j}($.$) are the individual utilities of each prosumer j \in \mathcal{N}(€)$

The dual variables of the energy management problem are not detailed in this nomenclature. However, they will be introduced in Appendix B, along with the constraints that they are associated with.

\section{Appendix A.2. Conventions}

In this paper we use the following conventions and mathematical operators. For any matrix $A=\left[A_{1}, . ., A_{J}\right] \in \mathbb{R}_{I \times J}$, we denote the column $A_{j} \in \mathbb{R}^{I}$ for any $j \in\{1, \ldots, J\}$. We define $A^{\top} \in \mathbb{R}_{J \times I}$ the transpose matrix, such that $A_{j, i}^{\top}=A_{i, j}, \quad \forall i \in\{1, \ldots, I\}, \forall j \in\{1, \ldots, J\}$. We define $A_{j} \in \mathbb{R}_{I}$ the vector of the $j^{\text {th }}$ column, and $(A)_{i}^{T} \in \mathbb{R}_{J}$ the vector of the $i^{\text {th }}$ line. The operators $\leq, \geq$, $=$, and $\perp$, are used element-by-element. For instance, for $A \in \mathbb{R}_{I \times J}$ and $B \in \mathbb{R}_{I \times J}$, the inequality $A \leq B$ is understood as $A_{i, j} \leq B_{i, j}, \quad \forall i \in\{1, \ldots, I\}, \forall j \in\{1, \ldots, J\}$.

The Euclidean inner product between $A \in \mathbb{R}_{I \times K}$ and $B \in \mathbb{R}_{K \times J}$, is defined as $(A B)_{i, j}=$ $\sum_{k=1}^{K} A_{i, k} B_{k, j}$, for $i \in\{1, \ldots, I\}$ and $j \in\{1, \ldots, J\}$. In particular, the right-side product of matrix $A \in \mathbb{R}_{I \times T}$ with the column vector of ones $\mathbf{1}_{T}$ represents a sum over the rows of the matrix $A \in \mathbb{R}_{I \times T}$, and the left-side product with the row vector of ones $\mathbf{1}_{1 \times I}$ represents a sum of the columns of the matrix $A \in \mathbb{R}_{I \times T}$.

We denote $\circ$ as the Hadamard product between two matrices of the same dimensions $A, B \in$ $\mathbb{R}_{I \times J}$, such that $A \circ B=\left(A_{i, j} B_{i, j}\right)_{1 \leq i \leq I, 1 \leq j \leq J}$. By extension we define the Hadamard product between a matrix $A \in \mathbb{R}_{I \times J}$ and a column vector $\vec{B} \in \mathbb{R}_{I}$ as $A \circ \vec{B}=\left[A_{1} \circ \vec{B}, \ldots, A_{J} \circ \vec{B}\right] \in \mathbb{R}_{I \times J}$.

Furthermore, a matrix $Z=\left[Z_{1}, \ldots, Z_{N}\right] \in \mathbb{R}^{M \times N}$, with the columns $Z_{j} \in \mathbb{R}^{M}$ for $j \in\{1, \ldots, N\}$, indexed by a coalition $\mathcal{S} \in 2^{\mathcal{N}}$ is defined as $Z^{\mathcal{S}}=\left[Z_{1} \delta_{1}^{\mathcal{S}}, \ldots, Z_{N} \delta_{N}^{\mathcal{S}}\right]$. Following this definition, we introduce the summation operation of the coalition superscripts.

Definition 8 (Coalition summation). For a matrix $Z=\left[Z_{1}, \ldots, Z_{N}\right] \in \mathbb{R}^{M \times N}$, and the coalitions $\mathcal{S}_{k} \in 2^{\mathcal{N}}$, and $a_{k} \geq 0$ for $j \in\left\{1, \ldots, 2^{N}\right\}$ is defined as:

$$
Z^{\sum_{k=1}^{2^{N}} a_{k} \mathcal{S}_{k}}=\left[\sum_{k=1}^{2^{N}} a_{k} Z_{1} \delta_{1}^{\mathcal{S}_{k}}, \ldots, \sum_{k=1}^{2^{N}} a_{k} Z_{N} \delta_{N}^{\mathcal{S}_{k}}\right] .
$$


From (A.1), we can derive the following:

$$
Z^{\sum_{k=1}^{2^{N}} a_{k} \mathcal{S}_{k}}=\sum_{k=1}^{2^{N}}\left[a_{k} Z_{1} \delta_{1}^{\mathcal{S}_{k}}, \ldots, a_{k} Z_{k} \delta_{N}^{\mathcal{S}_{k}}\right]=\sum_{k=1}^{2^{N}} Z^{a_{k} \mathcal{S}_{k}}=\sum_{k=1}^{2^{N}} a_{k} Z^{\mathcal{S}_{k}}
$$

In particular, for $\mathcal{S} \in 2^{\mathcal{N}}, \mathcal{V} \in 2^{\mathcal{N}}$ such that $\mathcal{S} \cap \mathcal{V}=\emptyset$, we have $\delta_{j}^{\mathcal{S}}+\delta_{j}^{\mathcal{V}}=\delta_{j}^{\mathcal{S} \cup \mathcal{V}}, \forall j \in\{1, \ldots, N\}$. Hence, $Z^{\mathcal{S}}+Z^{\mathcal{V}}=Z^{\mathcal{S} \cup \mathcal{V}}$.

\section{Appendix B. Mathematical formulations}

This Appendix details the mathematical models representing the dynamics and constraints of the prosumers, and the energy management problem of the community manager.

\section{Appendix B.1. Temperature dynamics of buildings}

We introduce the following additional notations representing the technical characteristics of the buildings in the community:

$C_{j}^{a}$ Thermal capacity of the indoor air of a building $j \in \mathcal{N}\left(\mathrm{J} /{ }^{\circ} \mathrm{C}\right)$

$C_{j}^{\mathrm{s}}$ Thermal inertia of the fabric of a building $j \in \mathcal{N}\left(\mathrm{J} /{ }^{\circ} \mathrm{C}\right)$

$T_{t, j}^{\mathrm{e}}$ External outdoor air temperatures of a building $j \in \mathcal{N}$ at time $t \in \mathcal{T}\left({ }^{\circ} \mathrm{C}\right)$

$T_{j}^{\mathrm{g}}$ Ground temperature of a building $j \in \mathcal{N}\left({ }^{\circ} \mathrm{C}\right)$

$T_{t, j}^{\mathrm{v}}$ Ventilation supply air temperature of a building $j \in \mathcal{N}$ at time $t \in \mathcal{T}\left({ }^{\circ} \mathrm{C}\right)$

$H_{j}^{\text {ae }}$ Sum of the infiltration heat capacity flow and windows heat conductance $\left(\mathrm{W} /{ }^{\circ} \mathrm{C}\right)$

$H_{j}^{\text {as }}$ Sum of heat conductance in the solid wall and convection on the surface $\left(\mathrm{W} /{ }^{\circ} \mathrm{C}\right)$

$H_{j}^{\mathrm{ag}}$ Floor heat conductance $\left(\mathrm{W} /{ }^{\circ} \mathrm{C}\right)$

$H_{j}^{\text {av }}$ Ventilation air heat capacity flow $\left(\mathrm{W} /{ }^{\circ} \mathrm{C}\right)$

$H_{j}^{\text {se }}$ Sum of heat conductance in the solid wall and convection on the surface $\left(\mathrm{W} /{ }^{\circ} \mathrm{C}\right)$

$\Phi_{t, j}^{\mathrm{R}}$ Total heating power from solar radiation, and internal heat gains of a building $j \in \mathcal{N}$ at time $t \in \mathcal{T}(\mathrm{W})$

Note that every parameter depends on the building type except for $T_{t, j}^{\mathrm{e}}$ and $T_{j}^{\mathrm{g}}$, which depend on the location of the building. The parameter $\Phi_{t, j}^{\mathrm{R}}$ depends on the location and the building type.

Based on the set of differential equations introduced in (Alahäivälä et al., 2017) representing the temporal dynamics of the ambient room temperatures $T^{\mathrm{a}} \in \mathbb{R}^{T \times N}$, the building's fabric temperatures $T^{\mathrm{s}} \in \mathbb{R}^{T \times N}$, we can derive the following linear model:

$$
\begin{aligned}
& \alpha_{j}^{3} T_{t, j}^{\mathrm{a}}-T_{t-1, j}^{\mathrm{a}}=\alpha_{t, j}^{0}+\alpha_{j}^{1} T_{t, j}^{\mathrm{s}}+\alpha_{j}^{2} L_{t, j}^{\mathrm{SH}}, \quad \forall j \in \mathcal{N}, t \in \mathcal{T} \\
& \beta_{j}^{2} T_{t, j}^{\mathrm{s}}-T_{t-1, j}^{\mathrm{s}}=\beta_{t, j}^{0}+\beta_{j}^{1} T_{t, j}^{\mathrm{a}}, \quad \forall j \in \mathcal{N}, t \in \mathcal{T},
\end{aligned}
$$


where the parameters are defined as

$$
\begin{aligned}
& \alpha_{t, j}^{0}=\frac{\Delta t}{C_{j}^{\mathrm{a}}}\left(H_{j}^{\mathrm{ae}} T_{t, j}^{\mathrm{e}}+H_{j}^{\mathrm{ag}} T_{j}^{\mathrm{g}}+H_{j}^{\mathrm{av}} T_{t, j}^{\mathrm{v}}+\Phi_{t, j}^{\mathrm{R}}\right), \quad \forall j \in \mathcal{N}, t \in \mathcal{T} \\
& \alpha_{j}^{1}=\frac{\Delta t}{C_{j}^{\mathrm{a}}} H_{j}^{\mathrm{as}}, \quad \forall j \in \mathcal{N} \\
& \alpha_{j}^{2}=\frac{\Delta t}{C_{j}^{\mathrm{a}}}, \quad \forall j \in \mathcal{N} \\
& \alpha_{j}^{3}=1+\frac{\Delta t}{C_{j}^{\mathrm{a}}}\left(H_{j}^{\mathrm{ae}}+H_{j}^{\mathrm{as}}+H_{j}^{\mathrm{ag}}+H_{j}^{\mathrm{av}}\right) \\
& \beta_{t, j}^{0}=\frac{\Delta t}{C_{j}^{\mathrm{s}}} H_{j}^{\mathrm{se}} T_{t, j}^{\mathrm{e}}, \quad \forall j \in \mathcal{N}, t \in \mathcal{T} \\
& \beta_{j}^{1}=\frac{\Delta t}{C_{j}^{\mathrm{s}}} H_{j}^{\mathrm{as}}, \quad \forall j \in \mathcal{N} \\
& \beta_{j}^{2}=1+\frac{\Delta t}{C_{j}^{\mathrm{s}}}\left(H_{j}^{\mathrm{as}}+H_{j}^{\mathrm{se}}\right), \quad \forall j \in \mathcal{N},
\end{aligned}
$$

where $\Delta t$ represents the discretization of the time steps (in seconds). Equations (B.1) can be reformulated in a compact form as

$$
\begin{aligned}
& T^{\mathrm{a}}=A^{0}+\sum_{k=1}^{T} M^{(k)} T^{\mathrm{s}} \circ A^{1, k}+\sum_{k=1}^{T} M^{(k)} L^{\mathrm{SH}} \circ A^{2, k} \\
& T^{\mathrm{s}}=B^{0}+\sum_{k=1}^{T} M^{(k)} T^{\mathrm{a}} \circ B^{1, k}
\end{aligned}
$$

where the matrices $A^{0} \in \mathbb{R}^{T \times N}, A^{1, k} \in \mathbb{R}^{T \times N}, A^{2, k} \in \mathbb{R}^{T \times N}, B^{0} \in \mathbb{R}^{T \times N}$, and $B^{1, k} \in \mathbb{R}^{T \times N}$ are defined by

$$
\begin{aligned}
& A_{t, j}^{0}=\sum_{\tau=1}^{T} \frac{\alpha_{\tau, j}^{0}}{\left(\alpha_{j}^{3}\right)^{t+1-\tau}}+\frac{1}{\left(\alpha_{j}^{3}\right)^{T}} T_{j}^{\mathrm{a}, 0}, \quad \forall j \in \mathcal{N}, \forall t \in \mathcal{T} \\
& A_{t, j}^{1, k}=\frac{\alpha_{j}^{1}}{\left(\alpha_{j}^{3}\right)^{k}}, \quad \forall j \in \mathcal{N}, \forall k \in \mathcal{T}, \forall t \in \mathcal{T} \\
& A_{t, j}^{2, k}=\frac{\alpha_{j}^{2}}{\left(\alpha_{j}^{3}\right)^{k}}, \quad \forall j \in \mathcal{N}, \forall t \in \mathcal{T} \\
& B_{t, j}^{0}=\sum_{\tau=1}^{T} \frac{\beta_{\tau, j}^{0}}{\left(\beta_{j}^{2}\right)^{t+1-\tau}}+\frac{1}{\left(\beta_{j}^{2}\right)^{T}} T_{j}^{\mathrm{s}, 0}, \quad \forall j \in \mathcal{N}, \forall t \in \mathcal{T} \\
& B_{t, j}^{1, k}=\frac{\beta_{j}^{1}}{\left(\beta_{j}^{2}\right)^{k}}, \quad \forall j \in \mathcal{N}, \forall k \in \mathcal{T}, \forall t \in \mathcal{T},
\end{aligned}
$$


and, for any $k \in\{1, \ldots, T\}$ the lower-triangular matrix $M^{(k)} \in \mathbb{R}_{T \times T}$ is defined as

$$
M_{i, j}^{(k)}=\left\{\begin{array}{l}
1 \text { if } i=j-1+k \\
0 \text { otherwise }
\end{array}\right.
$$

such that $M^{(k)}=\mathbb{I}_{T \times T}$ is the identity matrix, and $\sum_{k=1}^{T} M^{(k)}=\mathbf{1}_{T \times T}^{\downarrow}$ is the lower triangular matrix which lower coefficients are all equal to 1 . The left-side product of a matrix with the matrix $M^{(k)}$ represents the selection of the elements of that matrix at time $T-k$.

\section{Appendix B.2. Energy management problem: extended formulation}

In this section we introduce an extended formulation of the energy management problem of the community manager introduced in Section 3.

\section{Appendix B.2.1. Decision variables}

For each prosumer $j \in \mathcal{N}$, we distinguish three main types of variable loads over all hours $t \in \mathcal{T}$ of the day, depending on the utility that is derived from them, rather than the energy carrier (district heating or electricity) consumed:

1. Space heating: $L_{j}^{\mathrm{SH}} \in \mathbb{R}^{T}$ is the energy needed at each hour to heat a building. This load can be supplied by district heating, or electricity (via heat pumps). In order to model the flexibility of space heating loads, the temperature dynamics of each building are represented through a set of linear equations detailed in Appendix B (Alahäivälä et al., 2017). The utility derived from space heating is defined with respect to a vector of temperature comfort level $T_{j}^{\text {set }} \in \mathbb{R}^{T}$, and vectors of upward and downward deviation range $\bar{u}_{j}^{\text {up }} \in \mathbb{R}_{+}^{T}, \bar{u}_{j}^{\text {down }} \in \mathbb{R}_{+}^{T}$.

2. Domestic hot water: $L_{j}^{\mathrm{HW}} \in \mathbb{R}^{T}$ is the energy needed at each hour in the form of domestic hot water. This load can be supplied either by district heating, assuming that the district heating supply temperature is sufficient, or by electricity (via heat pumps).

3. Heat pumps: $L_{j}^{\mathrm{HP}} \in \mathbb{R}^{T}$ designates the electricity consumption of heat pumps.

4. Electrical appliances: $L_{j}^{\mathrm{E}} \in \mathbb{R}^{T}$ designates all electrical loads that do not serve the purpose of supplying one of the two aforementioned loads, i.e., heat pumps are not included.

The space of decisions for each prosumer $j \in \mathcal{N}$ over all hours of the following day, includes their import and export of heat and electricity from the grids $b_{j}^{\mathrm{H}} \in \mathbb{R}_{+}^{T}, b_{j}^{\mathrm{E}} \in \mathbb{R}_{+}^{T}, s_{j}^{\mathrm{H}} \in \mathbb{R}_{+}^{T}, s_{j}^{\mathrm{E}} \in \mathbb{R}_{+}^{T}$, the exchange of heat and electricity within the community $f_{j}^{\mathrm{H}} \in \mathbb{R}^{T}, f_{j}^{\mathrm{E}} \in \mathbb{R}^{T}$, flexible loads, electricity production from renewable energy $\Phi_{j}^{\mathrm{ENR}} \in \mathbb{R}^{T}$, heat and electricity production from thermal units $\Phi_{j}^{\mathrm{H}} \in \mathbb{R}_{+}^{T}, \Phi_{j}^{\mathrm{E}} \in \mathbb{R}_{+}^{T}$, charging and discharging of the heat and electricity storage units $c h_{j}^{\mathrm{E}} \in \mathbb{R}_{+}^{T}$, $d i s_{j}^{\mathrm{E}} \in \mathbb{R}_{+}^{T}, c h_{j}^{\mathrm{H}} \in \mathbb{R}_{+}^{T}, d i s_{j}^{\mathrm{H}} \in \mathbb{R}_{+}^{T}$. For notational simplicity, we assume that each prosumer has at most one of each type of generation or storage units. Furthermore, a set of state variables defined over all hours $t \in \mathcal{T}$ of the day, including the state of charge of heat and electricity storage units $e n_{j}^{\mathrm{E}} \in \mathbb{R}_{+}^{T}$, $e n_{j}^{\mathrm{H}} \in \mathbb{R}_{+}^{T}$, the indoor and fabric temperatures of the building $T_{j}^{\mathrm{a}} \in \mathbb{R}_{+}^{T}, T_{j}^{\mathrm{s}} \in \mathbb{R}_{+}^{T}$, upward and downward deviations from the temperature comfort level $u_{j}^{\text {up }} \in \mathbb{R}_{+}^{T}, u_{j}^{\text {down }} \in \mathbb{R}_{+}^{T}$, represents the state of the prosumer and may influence its utility. This set of variables for all prosumers in the community and over all hours of the planning horizon is denoted by the matrix $X=\left[X_{1}, \ldots, X_{N}\right] \in \mathbb{R}^{K_{2} \times N}$, where each column

$$
\begin{aligned}
X_{j}= & {\left[b_{j}^{\mathrm{H}}, b_{j}^{\mathrm{E}}, s_{j}^{\mathrm{H}}, s_{j}^{\mathrm{E}}, f_{j}^{\mathrm{H}}, f_{j}^{\mathrm{E}}, \Phi_{j}^{\mathrm{H}}, \Phi_{j}^{\mathrm{E}}, L_{j}^{\mathrm{SH}}, L_{j}^{\mathrm{HW}}, L_{j}^{\mathrm{E}}, u_{j}^{\mathrm{up}}, u_{j}^{\mathrm{down}}, T_{j}^{\mathrm{s}}, T_{j}^{\mathrm{a}}, e n_{j}^{\mathrm{E}}, e n_{j}^{\mathrm{H}},\right.} \\
& \left.c h_{j}^{\mathrm{E}}, d i s_{j}^{\mathrm{E}}, e n_{j}^{\mathrm{H}}, c h_{j}^{\mathrm{H}}, d i s_{j}^{\mathrm{H}}\right] \in \mathbb{R}^{K_{2}}
\end{aligned}
$$


represents the decision variables of a prosumer $j \in \mathcal{N}$ over all hours of the following day. 


\section{Appendix B.2.2. Community manager problem}

The energy management problem of a coalition $\mathcal{S} \in 2^{\mathcal{N}}$ solved by the community manager with respect to the revealed preferences $\mathcal{B}$ can be formulated as

$$
\begin{aligned}
\max [ & \mathbf{1}_{1 \times T}\left(L^{\mathrm{E}} \circ c^{\mathrm{E}}+L^{\mathrm{HW}} \circ c^{\mathrm{HW}}-u^{\mathrm{up}} \circ c^{\mathrm{up}}-u^{\mathrm{down}} \circ c^{\mathrm{down}}-\Phi^{\mathrm{H}} \circ c^{\mathrm{g}, \mathrm{H}}\right. \\
& \left.\left.-\Phi^{\mathrm{E}} \circ c^{\mathrm{g}, \mathrm{E}}\right)+c^{\mathrm{s}, \mathrm{E}^{\top}} s^{\mathrm{E}}+c^{\mathrm{s}, \mathrm{H}^{\top}} s^{\mathrm{H}}-c^{\mathrm{b}, \mathrm{E}^{\top}} b^{\mathrm{E}}-c^{\mathrm{b}, \mathrm{H}^{\top}} b^{\mathrm{H}}\right] \mathbf{1}_{N}
\end{aligned}
$$

s.t. $(4 \mathrm{a})-(4 \mathrm{~b}): \quad \lambda^{\mathrm{E}}, \lambda^{\mathrm{H}}$

$\Phi^{\mathrm{ENR}}+\Phi^{\mathrm{E}}-L^{\mathrm{E}}-L^{\mathrm{HP}}+d i s^{\mathrm{E}}-c h^{\mathrm{E}}=f^{\mathrm{E}}+s^{\mathrm{E}}-b^{\mathrm{E}}: \quad \gamma^{\mathrm{E}}$

$\Phi^{\mathrm{H}}+L^{\mathrm{HP}} \circ C O P-L^{\mathrm{SH}}-L^{\mathrm{HW}}+d i s^{\mathrm{H}}-c h^{\mathrm{H}}=f^{\mathrm{H}}+s^{\mathrm{H}}-b^{\mathrm{H}}: \gamma^{\mathrm{H}}$

$T^{\mathrm{a}}=A^{0^{\mathcal{S}}}+\sum_{k=1}^{T} M^{(k)}\left(T^{\mathrm{s}} \circ A^{1, k}\right)+\sum_{k=1}^{T} M^{(k)}\left(L^{\mathrm{SH}} \circ A^{2, k}\right): \quad \gamma^{T^{\mathrm{a}}}$

$T^{\mathrm{a}}=T^{\mathrm{set}^{\mathcal{S}}}+u^{\mathrm{up}}-u^{\text {down }}: \gamma^{T^{\mathrm{set}}}$

$T^{\mathrm{s}}=B^{0^{\mathcal{S}}}+\sum_{k=1}^{T} M^{(k)}\left(T^{\mathrm{a}} \circ B^{1, k}\right): \quad \gamma^{T^{\mathrm{s}}}$

$e n^{\mathrm{E}}=e n^{\mathrm{E}, 0^{\mathcal{S}}}+\mathbf{1}_{T \times T}^{\downarrow}\left(c h^{\mathrm{E}} \circ \rho^{\mathrm{ch}, \mathrm{E}}-d i s^{\mathrm{E}} \circ \rho^{\mathrm{dis}, \mathrm{E}}\right): \gamma^{e n^{\mathrm{E}}}$

$e n^{\mathrm{H}}=e n^{\mathrm{H}, 0^{\mathcal{S}}}+\mathbf{1}_{T \times T}^{\downarrow}\left(c h^{\mathrm{H}} \circ \rho_{j}^{\mathrm{ch}, \mathrm{H}}-d i s^{\mathrm{H}} \circ \rho^{\mathrm{dis}, \mathrm{H}}\right): \quad \gamma^{e n^{\mathrm{H}}}$

$e n_{T}^{\mathrm{E}} \geq e n_{T}^{\mathrm{E}, 0^{\mathcal{S}}}: \mu^{\mathrm{en}, \mathrm{E}^{0}}$

$e n_{T}^{\mathrm{H}} \geq e n_{T}^{\mathrm{H}, 0^{\mathcal{S}}}: \mu^{\mathrm{en}, \mathrm{H}^{0}}$

$\Phi^{\mathrm{E}} \circ \rho^{\mathrm{E}}+\Phi_{j}^{\mathrm{H}} \circ \rho^{\mathrm{H}} \leq \bar{F}^{\mathcal{S}}: \quad \bar{\mu}^{F}$

$\Phi_{j}^{\mathrm{H}} \circ r \leq \Phi^{\mathrm{E}}: \quad \mu^{r}$

$\mathbf{0}_{T \times N} \leq b^{\mathrm{E}} \leq \bar{b}^{\mathrm{E}^{\mathcal{S}}}, \mathbf{0}_{T \times N} \leq s^{\mathrm{E}} \leq \bar{s}^{\mathrm{E}^{\mathcal{S}}}: \quad \underline{\mu}^{b^{\mathrm{E}}}, \bar{\mu}^{b^{\mathrm{E}}}, \underline{\mu}^{s^{\mathrm{E}}}, \bar{\mu}^{s^{\mathrm{E}}}$

$\mathbf{0}_{T \times N} \leq b^{\mathrm{H}} \leq \bar{b}^{\mathrm{H}^{\mathcal{S}}}, \mathbf{0}_{T \times N} \leq s^{\mathrm{H}} \leq \bar{s}^{\mathrm{H}^{\mathcal{S}}}: \quad \underline{\mu}^{b^{\mathrm{H}}}, \bar{\mu}^{b^{\mathrm{H}}}, \underline{\mu}^{s^{\mathrm{H}}}, \bar{\mu}^{s^{\mathrm{H}}}$

$-\bar{f}^{\mathrm{E}^{\mathcal{S}}} \leq f^{\mathrm{E}} \leq \bar{f}^{\mathrm{E}^{\mathcal{S}}},-\bar{f}^{\mathrm{H}^{\mathcal{S}}} \leq f^{\mathrm{H}} \leq \bar{f}^{\mathrm{H}^{\mathcal{S}}}: \quad \underline{\mu}^{\mathrm{f}, \mathrm{E}}, \bar{\mu}^{\mathrm{f}, \mathrm{E}}, \mu^{\mathrm{f}, \mathrm{H}}, \bar{\mu}^{\mathrm{f}, \mathrm{H}}$

$\mathbf{0}_{T \times N} \leq \Phi^{\mathrm{E}} \leq \bar{\Phi}^{\mathrm{E}^{\mathcal{S}}}, \mathbf{0}_{T \times N} \leq \Phi^{\mathrm{H}} \leq \bar{\Phi}^{\mathrm{H}^{\mathcal{S}}}: \quad \underline{\mu}^{\Phi^{\mathrm{E}}}, \bar{\mu}^{\Phi^{\mathrm{E}}}, \underline{\mu}^{\Phi^{\mathrm{H}}}, \bar{\mu}^{\Phi^{\mathrm{H}}}$

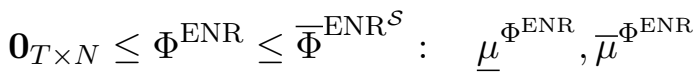

$\mathbf{0}_{T \times N} \leq u^{\mathrm{up}} \leq \bar{u}^{\mathrm{up}}{ }^{\mathcal{S}}, \mathbf{0}_{T \times N} \leq u^{\text {down }} \leq \bar{u}^{\mathrm{down}}{ }^{\mathcal{S}}: \quad \underline{\mu}^{u^{\text {up }}}, \bar{\mu}^{u^{\text {up }}}, \underline{\mu}^{u^{\text {down }}}, \bar{\mu}^{u^{\text {down }}}$

$\mathbf{0}_{T \times N} \leq L^{\mathrm{E}} \leq \bar{L}^{\mathrm{E}^{\mathcal{S}}}, \mathbf{0}_{T \times N} \leq L^{\mathrm{HP}} \leq \bar{L}^{\mathrm{HP} \mathcal{S}}: \quad \underline{\mu}^{L^{\mathrm{E}}}, \bar{\mu}^{L^{\mathrm{E}}}, \underline{\mu}^{L^{\mathrm{HP}}}, \bar{\mu}^{L^{\mathrm{HP}}}$

$\mathbf{0}_{T \times N} \leq L^{\mathrm{HW}} \leq \bar{L}^{\mathrm{HW}}{ }^{\mathcal{S}}, \mathbf{0}_{T \times N} \leq L^{\mathrm{SH}} \leq \bar{L}^{\mathrm{SH}}{ }^{\mathcal{S}}: \quad \mu^{L^{\mathrm{HW}}}, \bar{\mu}^{L^{\mathrm{HW}}}, \mu^{L^{\mathrm{SH}}}, \bar{\mu}^{L^{\mathrm{SH}}}$

$\mathbf{0}_{T \times N} \leq e n^{\mathrm{E}} \leq \overline{e n}^{\mathrm{E}^{\mathcal{S}}}, \mathbf{0}_{T \times N} \leq e n^{\mathrm{H}} \leq \overline{e n} \mathrm{H}^{\mathcal{S}}: \quad \mu^{e n^{\mathrm{E}}}, \bar{\mu}^{e n^{\mathrm{E}}}, \mu^{e n^{\mathrm{H}}}, \bar{\mu}^{e n^{\mathrm{H}}}$

$\mathbf{0}_{T \times N} \leq c h^{\mathrm{E}} \leq \overline{c h}^{\mathrm{E}^{\mathcal{S}}}, \mathbf{0}_{T \times N} \leq c h^{\mathrm{H}} \leq \overline{c h}^{\mathrm{H}^{\mathcal{S}}}: \quad \underline{\mu}^{c h^{\mathrm{E}}}, \bar{\mu}^{c h^{\mathrm{E}}}, \underline{\mu}^{c h^{\mathrm{H}}}, \bar{\mu}^{c h^{\mathrm{H}}}$

$\mathbf{0}_{T \times N} \leq d i s^{\mathrm{E}} \leq \overline{d i s}^{\mathrm{E}^{\mathcal{S}}}, \mathbf{0}_{T \times N} \leq d i s^{\mathrm{H}} \leq \overline{d i s}{ }^{\mathrm{H}^{\mathcal{S}}}: \quad \underline{\mu}^{d i s^{\mathrm{E}}}, \bar{\mu}^{d i s^{\mathrm{E}}}, \underline{\mu}^{d i s^{\mathrm{H}}}, \bar{\mu}^{d i s^{\mathrm{H}}}$. 
The dual variables are denoted in front of the constraints they are associated with. Equation (B.6b) represents the heat and electricity balance for the community, and the associated dual variables represent the heat and electricity uniform prices introduced in Section 3. Equations (B.6c) and (B.6d) represent the heat and electricity balance of individual prosumers in the community, (B.6e)-(B.6g) model the temperature dynamics of the buildings, (B.6h)-(B.6k) model the dynamics and state of charge of the heat and electricity storage units, and (B.6n)-(B.6x) represent the upper bounds for all decision variables. Furthermore, (B.6l) and (B.6m) represent a general formulation of the linking constraints between the heat and electricity outputs of thermal units, which encompass extraction CHPs, heat-only, and thermal power plants. In particular, (B.6l) represents the maximum fuel consumption, as a function of heat and electricity outputs, and (B.6m) represents the minimum heat to power ratio of thermal units.

This linear optimization problem may be reformulated in the compact form (7) by introducing the matrix $Q^{\mathrm{c}}=\left[Q_{1}^{\mathrm{c}}, \ldots, Q_{N}^{\mathrm{c}}\right] \in \mathbb{R}^{K_{4} \times N}$, which columns represent the load utilities and production costs of the prosumers over all hours $t \in \mathcal{T}$ of the day, i.e.,

$$
Q_{j}^{\mathrm{c}}=\left[c_{j}^{\mathrm{E}}, c_{j}^{\mathrm{HW}}, c_{j}^{\mathrm{up}}, c_{j}^{\mathrm{down}}, c_{j}^{\mathrm{g}, \mathrm{E}}, c_{j}^{\mathrm{g}, \mathrm{H}}\right] \in \mathbb{R}^{K_{4}}, \quad \forall j \in \mathcal{N},
$$

and the matrices $Q^{(0)} \in \mathbb{R}^{K_{3} \times N}, Q^{(1)} \in \mathbb{R}^{K_{2} \times N}, Q^{(2)} \in \mathbb{R}^{K_{3} \times K_{2}}$ which represent the feasible region of the decision variables $X \in \mathbb{R}^{K_{2} \times N}$ over all hours $t \in \mathcal{T}$ of the day. These matrices of technical parameters can be directly derived from the expression of the constraints (B.6c)-(B.6x).

Furthermore, the objective function (B.6a) can be separated for each prosumer $j \in \mathcal{N}$ into the element that depend on their bids $Q_{j}^{\mathrm{c}}$, i.e.,

$$
\begin{gathered}
\mathcal{F}_{j}\left(X_{j}, Q_{j}^{\mathrm{c}}\right)=\mathbf{1}_{1 \times T}\left(L_{j}^{\mathrm{E}} \circ c_{j}^{\mathrm{E}}+L_{j}^{\mathrm{HW}} \circ c_{j}^{\mathrm{HW}}-u_{j}^{\mathrm{up}} \circ c_{j}^{\mathrm{up}}-u_{j}^{\text {down }} \circ c_{j}^{\text {down }}\right. \\
\left.-\Phi_{j}^{\mathrm{H}} \circ c_{j}^{\mathrm{g}, \mathrm{H}}-\Phi_{j}^{\mathrm{E}} \circ c_{j}^{\mathrm{g}, \mathrm{E}}\right), \quad \forall j \in \mathcal{N},
\end{gathered}
$$

and the additional revenue from exchanges with the grid, i.e.,

$$
\mathcal{G}_{j}\left(X_{j}\right)=c_{j}^{\mathrm{s}, \mathrm{E}^{\top}} s_{j}^{\mathrm{E}}+c_{j}^{\mathrm{s}, \mathrm{H}^{\top}} s_{j}^{\mathrm{H}}-c_{j}^{\mathrm{b}, \mathrm{E}^{\top}} b_{j}^{\mathrm{E}}-c_{j}^{\mathrm{b}, \mathrm{H}^{\top}} b_{j}^{\mathrm{H}}, \quad \forall j \in \mathcal{N} .
$$

Appendix B.3. Energy management problem: Karush-Kuhn-Tucker (KKT) conditions

In the following we provide a detailed expression of the KKT conditions of the energy management problem (B.6). This formulation will be used to demonstrate the equivalence between this optimization problem and an equilibrium problem, where each (price-taker) agent tries to maximize its own profit in the community.

As the energy management problem (B.6) is linear in the continuous variables $X \in \mathbb{R}^{K_{2} \times N}$, it 
is equivalent to its KKT conditions, which can be expressed as

$$
\begin{aligned}
& \frac{\partial \mathcal{L}}{\partial f_{t, j}^{\mathrm{E}}}=-\lambda_{t, j}^{\mathrm{E}}+\gamma_{t, j}^{\mathrm{E}}-\underline{\mu}_{t, j}^{f^{\mathrm{E}}}+\bar{\mu}_{t, j}^{f^{\mathrm{E}}}=0, \quad \forall j \in \mathcal{N}, t \in \mathcal{T} \\
& \frac{\partial \mathcal{L}}{\partial f_{t, j}^{\mathrm{H}}}=-\lambda_{t, j}^{\mathrm{H}}+\gamma_{t, j}^{\mathrm{H}}-\underline{\mu}_{t, j}^{f^{\mathrm{H}}}+\bar{\mu}_{t, j}^{f^{\mathrm{H}}}=0, \quad \forall j \in \mathcal{N}, t \in \mathcal{T} \\
& \frac{\partial \mathcal{L}}{\partial b_{t, j}^{\mathrm{E}}}=c_{t}^{\mathrm{b}, \mathrm{E}}-\gamma_{t, j}^{\mathrm{E}}-\underline{\mu}_{t, j}^{b^{\mathrm{E}}}+\bar{\mu}_{t, j}^{b^{\mathrm{E}}}=0, \quad \forall j \in \mathcal{N}, t \in \mathcal{T} \\
& \frac{\partial \mathcal{L}}{\partial b_{t, j}^{\mathrm{H}}}=c_{t}^{\mathrm{b}, \mathrm{H}}-\gamma_{t, j}^{\mathrm{H}}-\underline{\mu}_{t, j}^{b^{\mathrm{H}}}+\bar{\mu}_{t, j}^{b^{\mathrm{H}}}=0, \quad \forall j \in \mathcal{N}, t \in \mathcal{T} \\
& \frac{\partial \mathcal{L}}{\partial s_{t, j}^{\mathrm{E}}}=-c_{t}^{\mathrm{s}, \mathrm{E}}+\gamma_{t, j}^{\mathrm{E}}-\underline{\mu}_{t, j}^{s^{\mathrm{E}}}+\bar{\mu}_{t, j}^{s^{\mathrm{E}}}=0, \quad \forall j \in \mathcal{N}, t \in \mathcal{T} \\
& \frac{\partial \mathcal{L}}{\partial s_{t, j}^{\mathrm{H}}}=-c_{t}^{\mathrm{s}, \mathrm{H}}+\gamma_{t, j}^{\mathrm{H}}-\underline{\mu}_{t, j}^{s^{\mathrm{H}}}+\bar{\mu}_{t, j}^{s^{\mathrm{H}}}=0, \quad \forall j \in \mathcal{N}, t \in \mathcal{T} \\
& \frac{\partial \mathcal{L}}{\partial \Phi_{t, j}^{\mathrm{E}}}=c_{j}^{\mathrm{g}, \mathrm{E}}-\gamma_{t, j}^{\mathrm{E}}-\underline{\mu}_{t, j}^{\Phi^{\mathrm{E}}}+\bar{\mu}_{t, j}^{\Phi^{\mathrm{E}}}+\bar{\mu}_{t, j}^{F} \rho_{t, j}^{\mathrm{E}}-\mu_{t, j}^{r}=0, \quad \forall j \in \mathcal{N}, t \in \mathcal{T} \\
& \frac{\partial \mathcal{L}}{\partial \Phi_{t, j}^{\mathrm{H}}}=c_{j}^{\mathrm{g}, \mathrm{H}}-\gamma_{t, j}^{\mathrm{H}}-\underline{\mu}_{t, j}^{\Phi^{\mathrm{H}}}+\bar{\mu}_{t, j}^{\Phi^{\mathrm{H}}}+\bar{\mu}_{t, j}^{F} \rho_{t, j}^{\mathrm{H}}+\mu_{t, j}^{r} r_{t, j}=0, \quad \forall j \in \mathcal{N}, t \in \mathcal{T} \\
& \frac{\partial \mathcal{L}}{\partial \Phi_{t, j}^{\mathrm{ENR}}}=-\gamma_{t, j}^{\mathrm{E}}-\underline{\mu}_{t, j}^{\Phi^{\mathrm{ENR}}}+\bar{\mu}_{t, j}^{\Phi^{\mathrm{ENR}}}=0, \quad \forall j \in \mathcal{N}, t \in \mathcal{T} \\
& \frac{\partial \mathcal{L}}{\partial L_{t, j}^{\mathrm{E}}}=-c_{t, j}^{\mathrm{E}}+\gamma_{t, j}^{\mathrm{E}}-\underline{\mu}_{t, j}^{L^{\mathrm{E}}}+\bar{\mu}_{t, j}^{L^{\mathrm{E}}}=0, \quad \forall j \in \mathcal{N}, t \in \mathcal{T} \\
& \frac{\partial \mathcal{L}}{\partial L_{t, j}^{\mathrm{HP}}}=\gamma_{t, j}^{\mathrm{E}}-C O P_{t, j} \gamma_{t, j}^{\mathrm{H}}+\bar{\mu}_{t, j}^{L^{\mathrm{HP}}}-\underline{\mu}_{t, j}^{L^{\mathrm{HP}}}=0, \quad \forall j \in \mathcal{N}, t \in \mathcal{T} \\
& \frac{\partial \mathcal{L}}{\partial L_{t, j}^{\mathrm{HW}}}=-c_{t, j}^{\mathrm{HW}}+\gamma_{t, j}^{\mathrm{H}}-\underline{\mu}_{t, j}^{L^{\mathrm{HW}}}+\bar{\mu}_{t, j}^{L^{\mathrm{HW}}}=0, \quad \forall j \in \mathcal{N}, t \in \mathcal{T} \\
& \frac{\partial \mathcal{L}}{\partial L_{t, j}^{\mathrm{SH}}}=\gamma_{t, j}^{\mathrm{H}}+\sum_{k, \tau=1}^{T} M_{\tau, t}^{(k)} A_{t, j}^{2, k} \gamma_{\tau, j}^{T^{\mathrm{a}}}-\underline{\mu}_{t, j}^{L^{\mathrm{SH}}}+\bar{\mu}_{t, j}^{L^{\mathrm{SH}}}=0, \quad \forall j \in \mathcal{N}, t \in \mathcal{T} \\
& \frac{\partial \mathcal{L}}{\partial e n_{t, j}^{\mathrm{E}}}=-\gamma_{t, j}^{e n^{\mathrm{E}}}-\underline{\mu}_{t, j}^{e n^{\mathrm{E}}}+\bar{\mu}_{t, j}^{e n^{\mathrm{E}}}-\mu^{\mathrm{en}, \mathrm{E}^{0}} \delta_{t}^{T}=0, \quad \forall j \in \mathcal{N}, t \in \mathcal{T} \\
& \frac{\partial \mathcal{L}}{\partial e n_{t, j}^{\mathrm{H}}}=-\gamma_{t, j}^{e n^{\mathrm{H}}}-\underline{\mu}_{t, j}^{e n^{\mathrm{H}}}+\bar{\mu}_{t, j}^{e n^{\mathrm{H}}}-\mu^{\mathrm{en}, \mathrm{H}^{0}} \delta_{t}^{T}=0, \quad \forall j \in \mathcal{N}, t \in \mathcal{T} \\
& \frac{\partial \mathcal{L}}{\partial c h_{t, j}^{\mathrm{E}}}=\gamma_{t, j}^{\mathrm{E}}+\sum_{\tau=1}^{T} \mathbf{1}_{\tau, t}^{\downarrow} \rho_{t, j}^{c h^{\mathrm{E}}} \gamma_{\tau, j}^{e n^{\mathrm{E}}}-\underline{\mu}_{t, j}^{c h^{\mathrm{E}}}+\bar{\mu}_{t, j}^{c h}=0, \quad \forall j \in \mathcal{N}, t \in \mathcal{T} \\
& \frac{\partial \mathcal{L}}{\partial c h_{t, j}^{\mathrm{H}}}=\gamma_{t, j}^{\mathrm{H}}+\sum_{\tau=1}^{T} \mathbf{1}_{\tau, t}^{\downarrow} \rho_{t, j}^{c h^{\mathrm{H}}} \gamma_{\tau, j}^{e n^{\mathrm{H}}}-\underline{\mu}_{t, j}^{c h^{\mathrm{H}}}+\bar{\mu}_{t, j}^{c h}=0, \quad \forall j \in \mathcal{N}, t \in \mathcal{T}
\end{aligned}
$$




$$
\begin{aligned}
& \frac{\partial \mathcal{L}}{\partial d i s_{t, j}^{\mathrm{E}}}=-\gamma_{t, j}^{\mathrm{E}}-\sum_{\tau=1}^{T} \mathbf{1}_{\tau, t}^{\downarrow} \rho_{t, j}^{d i s^{\mathrm{E}}} \gamma_{\tau, j}^{e n^{\mathrm{E}}}-\underline{\mu}_{t, j}^{d i s^{\mathrm{E}}}+\bar{\mu}_{t, j}^{d i s^{\mathrm{E}}}=0, \quad \forall j \in \mathcal{N}, t \in \mathcal{T} \\
& \frac{\partial \mathcal{L}}{\partial d i s_{t, j}^{\mathrm{H}}}=-\gamma_{t, j}^{\mathrm{H}}-\sum_{\tau=1}^{T} \mathbf{1}_{\tau, t}^{\downarrow} \rho_{t, j}^{d i s^{\mathrm{H}}} \gamma_{\tau, j}^{e n^{\mathrm{H}}}-\underline{\mu}_{t, j}^{d i s^{\mathrm{H}}}+\bar{\mu}_{t, j}^{d i s^{\mathrm{H}}}=0, \quad \forall j \in \mathcal{N}, t \in \mathcal{T} \\
& \frac{\partial \mathcal{L}}{\partial T_{t, j}^{\mathrm{a}}}=-\gamma_{t, j}^{T^{\mathrm{set}}}-\gamma_{t, j}^{T^{\mathrm{a}}}+\sum_{k, \tau=1}^{T} M_{\tau, t}^{(k)} B_{t, j}^{1, k} \gamma_{\tau, j}^{T^{\mathrm{s}}}=0, \quad \forall j \in \mathcal{N}, t \in \mathcal{T} \\
& \frac{\partial \mathcal{L}}{\partial T_{t, j}^{\mathrm{s}}}=-\gamma_{t, j}^{T^{\mathrm{s}}}+\sum_{k, \tau=1}^{T} M_{\tau, t}^{(k)} A_{t, j}^{1, k} \gamma_{\tau, j}^{T^{\mathrm{a}}}-\underline{\mu}_{j, t}^{T^{\mathrm{s}}}+\bar{\mu}_{j, t}^{T^{\mathrm{s}}}=0, \quad \forall j \in \mathcal{N}, t \in \mathcal{T} \\
& \frac{\partial \mathcal{L}}{\partial u_{t, j}^{\mathrm{up}}}=+c_{t, j}^{\mathrm{up}}+\gamma_{t, j}^{T^{\mathrm{set}}}-\underline{\mu}_{j, t}^{u^{\mathrm{up}}}+\bar{\mu}_{j, t}^{u_{\mathrm{up}}^{\mathrm{up}}}=0, \quad \forall j \in \mathcal{N}, t \in \mathcal{T} \\
& \frac{\partial \mathcal{L}}{\partial u_{t, j}^{\text {down }}}=+c_{t, j}^{\text {down }}-\gamma_{t, j}^{T^{\text {set }}}-\underline{\mu}_{j, t}^{u^{\text {down }}}+\bar{\mu}_{j, t}^{u^{\text {down }}}=0, \quad \forall j \in \mathcal{N}, t \in \mathcal{T} \\
& \mathbf{0}_{T \times N} \leq \bar{\mu}^{F} \perp\left(\bar{F}^{\mathcal{S}}-\Phi^{\mathrm{E}} \circ \rho^{\mathrm{E}}-\Phi^{\mathrm{H}} \circ \rho^{\mathrm{H}}\right) \geq \mathbf{0}_{T \times N} \\
& \mathbf{0}_{T \times N} \leq \mu^{r} \perp\left(\Phi^{\mathrm{E}}-\Phi^{\mathrm{H}} \circ r\right) \geq \mathbf{0}_{T \times N} \\
& \mathbf{0}_{T \times N} \leq \underline{\mu}^{b^{\mathrm{E}}} \perp b^{\mathrm{E}} \geq \mathbf{0}_{T \times N}, \mathbf{0}_{T \times N} \leq \bar{\mu}^{b^{\mathrm{E}}} \perp\left(\bar{b}^{\mathrm{E}^{\mathcal{S}}}-b^{\mathrm{E}}\right) \geq \mathbf{0}_{T \times N} \\
& \mathbf{0}_{T \times N} \leq \underline{\mu}^{s^{\mathrm{E}}} \perp s^{\mathrm{E}} \geq \mathbf{0}_{T \times N}, \mathbf{0}_{T \times N} \leq \bar{\mu}^{s^{\mathrm{E}}} \perp\left(\bar{s}^{\mathrm{E}^{\mathcal{S}}}-s^{\mathrm{E}}\right) \geq \mathbf{0}_{T \times N} \\
& \mathbf{0}_{T \times N} \leq \underline{\mu}^{b^{\mathrm{H}}} \perp b^{\mathrm{H}} \geq \mathbf{0}_{T \times N}, \mathbf{0}_{T \times N} \leq \bar{\mu}^{b^{\mathrm{H}}} \perp\left(\bar{b}^{\mathrm{H}^{\mathcal{S}}}-b^{\mathrm{H}}\right) \geq \mathbf{0}_{T \times N} \\
& \mathbf{0}_{T \times N} \leq \underline{\mu}^{s^{\mathrm{H}}} \perp s^{\mathrm{H}} \geq \mathbf{0}_{T \times N}, \mathbf{0}_{T \times N} \leq \bar{\mu}^{s^{\mathrm{H}}} \perp\left(\bar{s}^{\mathrm{H}^{\mathcal{S}}}-s^{\mathrm{H}}\right) \geq \mathbf{0}_{T \times N} \\
& \mathbf{0}_{T \times N} \leq \underline{\mu}^{\mathrm{f}, \mathrm{E}} \perp\left(\bar{f}^{\mathrm{E}^{\mathcal{S}}}+f^{\mathrm{E}}\right) \geq \mathbf{0}_{T \times N}, \mathbf{0}_{T \times N} \leq \bar{\mu}^{\mathrm{f}, \mathrm{E}} \perp\left(\bar{f}^{\mathrm{E}^{\mathcal{S}}}-f^{\mathrm{E}}\right) \geq \mathbf{0}_{T \times N} \\
& \mathbf{0}_{T \times N} \leq \underline{\mu}^{\mathrm{f}, \mathrm{H}} \perp\left(\bar{f}^{\mathrm{H}^{\mathcal{S}}}+f^{\mathrm{H}}\right) \geq \mathbf{0}_{T \times N}, \mathbf{0}_{T \times N} \leq \bar{\mu}^{\mathrm{f}, \mathrm{H}} \perp\left(\bar{f}^{\mathrm{H}^{\mathcal{S}}}-f^{\mathrm{H}}\right) \geq \mathbf{0}_{T \times N} \\
& \mathbf{0}_{T \times N} \leq \underline{\mu}^{\Phi^{\mathrm{E}}} \perp \Phi^{\mathrm{E}} \geq \mathbf{0}_{T \times N}, \mathbf{0}_{T \times N} \leq \bar{\mu}^{\Phi^{\mathrm{E}}} \perp\left(\bar{\Phi}^{\mathrm{E}^{\mathcal{S}}}-\Phi^{\mathrm{E}}\right) \geq \mathbf{0}_{T \times N} \\
& \mathbf{0}_{T \times N} \leq \underline{\mu}^{\Phi^{\mathrm{H}}} \perp \Phi^{\mathrm{H}} \geq \mathbf{0}_{T \times N}, \mathbf{0}_{T \times N} \leq \bar{\mu}^{\Phi^{\mathrm{H}}} \perp\left(\bar{\Phi}^{\mathrm{H}^{\mathcal{S}}}-\Phi^{\mathrm{H}}\right) \geq \mathbf{0}_{T \times N} \\
& \mathbf{0}_{T \times N} \leq \underline{\mu}^{\Phi^{\mathrm{ENR}}} \perp \Phi^{\mathrm{ENR}} \geq \mathbf{0}_{T \times N}, \mathbf{0}_{T \times N} \leq \bar{\mu}^{\Phi^{\mathrm{ENR}}} \perp\left(\bar{\Phi}^{\mathrm{ENR}^{\mathcal{S}}}-\Phi^{\mathrm{ENR}}\right) \geq \mathbf{0}_{T \times N} \\
& \mathbf{0}_{T \times N} \leq \underline{\mu}^{u^{\mathrm{up}}} \perp u^{\mathrm{up}} \geq \mathbf{0}_{T \times N}, \mathbf{0}_{T \times N} \leq \bar{\mu}^{u^{\mathrm{up}}} \perp\left(\bar{u}^{\mathrm{up}}{ }^{\mathcal{S}}-u^{\mathrm{up}}\right) \geq \mathbf{0}_{T \times N} \\
& \mathbf{0}_{T \times N} \leq \underline{\mu}^{u^{\text {down }}} \perp u^{\text {down }} \geq \mathbf{0}_{T \times N}, \mathbf{0}_{T \times N} \leq \bar{\mu}^{u^{\text {down }}} \perp\left(\bar{u}^{\text {down }}{ }^{\mathcal{S}}-u^{\text {down }}\right) \geq \mathbf{0}_{T \times N} \\
& \mathbf{0}_{T \times N} \leq \underline{\mu}^{L^{\mathrm{E}}} \perp L^{\mathrm{E}} \geq \mathbf{0}_{T \times N}, \mathbf{0}_{T \times N} \leq \bar{\mu}^{L^{\mathrm{E}}} \perp\left(\bar{L}^{\mathrm{E}^{\mathcal{S}}}-L^{\mathrm{E}}\right) \geq \mathbf{0}_{T \times N} \\
& \mathbf{0}_{T \times N} \leq \underline{\mu}^{L^{\mathrm{HP}}} \perp L^{\mathrm{HP}} \geq \mathbf{0}_{T \times N}, \mathbf{0}_{T \times N} \leq \bar{\mu}^{L^{\mathrm{HP}}} \perp\left(\bar{L}^{\mathrm{HP}^{\mathcal{S}}}-L^{\mathrm{HP}}\right) \geq \mathbf{0}_{T \times N} \\
& \mathbf{0}_{T \times N} \leq \underline{\mu}^{L^{\mathrm{HW}}} \perp L^{\mathrm{HP}} \geq \mathbf{0}_{T \times N}, \mathbf{0}_{T \times N} \leq \bar{\mu}^{L^{\mathrm{HW}}} \perp\left(\bar{L}^{\mathrm{HW}^{\mathcal{S}}}-L^{\mathrm{HW}}\right) \geq \mathbf{0}_{T \times N}
\end{aligned}
$$




$$
\begin{aligned}
& \mathbf{0}_{T \times N} \leq \underline{\mu}^{L^{\mathrm{SH}}} \perp L^{\mathrm{SH}} \geq 0, \mathbf{0}_{T \times N} \leq \bar{\mu}^{L^{\mathrm{SH}}} \perp\left(\bar{L}^{\mathrm{SH}^{\mathcal{S}}}-L^{\mathrm{SH}}\right) \geq \mathbf{0}_{T \times N} \\
& \mathbf{0}_{T \times N} \leq \underline{\mu}^{e n^{\mathrm{E}}} \perp e n^{\mathrm{E}} \geq \mathbf{0}_{T \times N}, \mathbf{0}_{T \times N} \leq \bar{\mu}^{e n^{\mathrm{E}}} \perp\left(\overline{e n}^{\mathrm{E}^{\mathcal{S}}}-e n^{\mathrm{E}}\right) \geq \mathbf{0}_{T \times N} \\
& \mathbf{0}_{T} \leq \mu^{\mathrm{en}, \mathrm{E}^{0}} \perp\left(\left(e n_{T}^{\mathrm{E}}\right)^{\top}-\left(e n_{T}^{\mathrm{E}, 0^{\mathcal{S}}}\right)^{\top}\right) \geq \mathbf{0}_{T} \\
& \mathbf{0}_{T \times N} \leq \underline{\mu}^{e n^{\mathrm{H}}} \perp e n^{\mathrm{H}} \geq \mathbf{0}_{T \times N}, \mathbf{0}_{T \times N} \leq \bar{\mu}^{e n^{\mathrm{H}}} \perp\left(\overline{e n}^{\mathrm{H}^{\mathcal{S}}}-e n^{\mathrm{H}}\right) \geq \mathbf{0}_{T \times N} \\
& \mathbf{0}_{T} \leq \mu^{\mathrm{en}, \mathrm{H}^{0}} \perp\left(\left(e n_{T}^{\mathrm{H}}\right)^{\top}-\left(e n_{T}^{\mathrm{H}, 0^{\mathcal{S}}}\right)^{\top}\right) \geq \mathbf{0}_{T} \\
& \mathbf{0}_{T \times N} \leq \underline{\mu}^{c h^{\mathrm{E}}} \perp c h^{\mathrm{E}} \geq \mathbf{0}_{T \times N}, \mathbf{0}_{T \times N} \leq \bar{\mu}^{c h^{\mathrm{E}}} \perp\left(\overline{c h}^{\mathrm{E}^{\mathcal{S}}}-c h^{\mathrm{E}}\right) \geq \mathbf{0}_{T \times N} \\
& \mathbf{0}_{T \times N} \leq \underline{\mu}^{c h^{\mathrm{H}}} \perp c h^{\mathrm{H}} \geq \mathbf{0}_{T \times N}, \mathbf{0}_{T \times N} \leq \bar{\mu}^{c h^{\mathrm{H}}} \perp\left(\overline{c h} \overline{\mathrm{H}}^{\mathcal{S}}-c h^{\mathrm{H}}\right) \geq \mathbf{0}_{T \times N} \\
& \mathbf{0}_{T \times N} \leq \underline{\mu}^{d i s^{\mathrm{E}}} \perp d i s^{\mathrm{E}} \geq \mathbf{0}_{T \times N}, \mathbf{0}_{T \times N} \leq \bar{\mu}^{d i s^{\mathrm{E}}} \perp\left(\overline{d i s}^{\mathrm{E}}{ }^{\mathcal{S}}-d i s^{\mathrm{E}}\right) \geq \mathbf{0}_{T \times N} \\
& \mathbf{0}_{T \times N} \leq \underline{\mu}^{d i s^{\mathrm{H}}} \perp d i s^{\mathrm{H}} \geq \mathbf{0}_{T \times N}, \mathbf{0}_{T \times N} \leq \bar{\mu}^{d i s^{\mathrm{H}}} \perp\left(\overline{\operatorname{dis}}^{\mathrm{H}^{\mathcal{S}}}-d i s^{\mathrm{H}}\right) \geq \mathbf{0}_{T \times N} .
\end{aligned}
$$

\section{Appendix C. Proofs}

This Appendix presents the proofs of the properties introduced in this work.

\section{Appendix C.1. Proof of Proposition 1: super-additivity of value function}

In order to prove Proposition 1, we first formulate the following lemma:

Lemma 1. The optimal objective function of the energy management problem $\theta($.$) is super-additive,$ i.e., for any preferences $\mathcal{B} \in \mathbb{R}^{K_{1} \times N}$, set of coalitions $\mathcal{S} \in 2^{\mathcal{N}}$, and $\mathcal{V} \in 2^{\mathcal{N}}$, and positive real values $a \geq 0$ and $b \geq 0$,

$$
\theta^{a \mathcal{S}+b \mathcal{V}}(\mathcal{B}) \geq \theta^{a \mathcal{S}}(\mathcal{B})+\theta^{b \mathcal{V}}(\mathcal{B})
$$

\section{Appendix C.1.1. Proof of Lemma 1}

For any feasible solutions $X \in \Omega_{\mathcal{S}}(\mathcal{B})$ and $Y \in \Omega_{\mathcal{V}}(\mathcal{B})$, from the formulation of the constraints (B.6b)-(B.6x), it is straightforward that the matrix $a X+b Y$ is in the feasible space $\Omega_{(a \mathcal{S}+b \mathcal{V})}(\mathcal{B})$. In particular, for $X^{\mathcal{S}^{*}}(\mathcal{B}) \in \Omega_{\mathcal{S}}(\mathcal{B})$ and $X^{\mathcal{V}^{*}}(\mathcal{B}) \in \Omega_{\mathcal{V}}(\mathcal{B})$, we can derive that the optimal solution of the energy management problem over the coalition $(a \mathcal{S}+b \mathcal{V})$ satisfies

$$
\theta^{(a \mathcal{S}+b \mathcal{V})}(\mathcal{B})=\mathcal{H}\left(X^{(a \mathcal{S}+b \mathcal{V})^{*}}(\mathcal{B}), Q^{\mathrm{c}}\right) \geq \mathcal{H}\left(a X^{\mathcal{S}^{*}}(\mathcal{B})+b X^{\mathcal{V}^{*}}(\mathcal{B}), Q^{\mathrm{c}}\right) .
$$

As the objective function $\mathcal{H}\left(X, Q^{\mathrm{c}}\right)$ in $(\mathrm{B} .6 \mathrm{a})$ is a linear map, it results

$$
\theta^{(a \mathcal{S}+b \mathcal{V})}(\mathcal{B}) \geq a \mathcal{H}\left(X^{\mathcal{S}^{*}}(\mathcal{B}), Q^{\mathrm{c}}\right)+b \mathcal{H}\left(X^{\mathcal{V}^{*}}(\mathcal{B}), Q^{\mathrm{c}}\right)=a \theta^{\mathcal{S}}(\mathcal{B})+b \theta^{\mathcal{V}}(\mathcal{B})
$$




\section{Appendix C.1.2. Proof of Proposition 1}

Let us consider $\mathcal{B} \in \mathbb{R}^{K_{1} \times N}, \mathcal{S} \in 2^{\mathcal{N}}, \mathcal{V} \in 2^{\mathcal{N}}$, such that $\mathcal{S} \cap \mathcal{V}=\emptyset$, and $a \geq 0, b \geq 0$, and let us prove that $v^{\mathcal{S} \cup \mathcal{V}}(\mathcal{B}) \geq v^{\mathcal{S}}(\mathcal{B})+v^{\mathcal{V}}(\mathcal{B})$. It results from the summation operation on coalition superscripts in the special case where $\mathcal{S} \cap \mathcal{V}=\emptyset$ that $\Omega_{\mathcal{S}+\mathcal{V}}(\mathcal{B})=\Omega_{\mathcal{S} \cup \mathcal{V}}(\mathcal{B})$, and $\theta^{\mathcal{S}+\mathcal{V}}(\mathcal{B})=$ $\theta^{\mathcal{S} \cup \mathcal{V}}(\mathcal{B})$. Thus,

$$
v^{\mathcal{S} \cup \mathcal{V}}(\mathcal{B})=\theta^{\mathcal{S} \cup \mathcal{V}}(\mathcal{B})-\sum_{j \in \mathcal{S} \cup \mathcal{V}} \theta^{\{j\}}(\mathcal{B})=\theta^{(\mathcal{S}+\mathcal{V})}(\mathcal{B})-\sum_{j \in \mathcal{S}} \theta^{\{j\}}(\mathcal{B})-\sum_{j \in \mathcal{V}} \theta^{\{j\}}(\mathcal{B}) .
$$

It follows from the super-additivity of the optimal objective value demonstrated in Lemma 1 that

$$
v^{\mathcal{S} \cup \mathcal{V}}(\mathcal{B}) \geq \theta^{\mathcal{S}}(\mathcal{B})-\theta^{\mathcal{V}}(\mathcal{B})-\sum_{j \in \mathcal{S}} \theta^{\{j\}}(\mathcal{B})-\sum_{j \in \mathcal{V}} \theta^{\{j\}}(\mathcal{B})=v^{\mathcal{S}}(\mathcal{B})+v^{\mathcal{V}}(\mathcal{B}) .
$$

Appendix C.2. Proof of Proposition 2: balancedness of cooperative game

Let us consider a balanced map $\alpha$, as introduced in Definition 7 , and the preferences $\mathcal{B} \in \mathbb{R}^{K_{1} \times N}$, and let us prove that the value function $v(\mathcal{B})$ defined in $(9)$ is balanced. By definition, the value function can be formulated as

$$
\begin{aligned}
\sum_{S \in 2^{\mathcal{N}}} \alpha(\mathcal{S}) v^{\mathcal{S}}(\mathcal{B}) & =\sum_{S \in 2^{\mathcal{N}}} \alpha(\mathcal{S})\left[\theta^{\mathcal{S}}(\mathcal{B})-\sum_{j \in \mathcal{S}} \theta^{\{j\}}(\mathcal{B})\right] \\
& =\sum_{S \in 2^{\mathcal{N}}} \alpha(\mathcal{S}) \theta^{\mathcal{S}}(\mathcal{B})-\sum_{j \in \mathcal{N}}\left(\sum_{S \in 2^{\mathcal{N}}} \alpha(\mathcal{S}) \delta_{j}^{\mathcal{S}}\right) \theta^{\{j\}}(\mathcal{B}) \\
& =\sum_{S \in 2^{\mathcal{N}}} \alpha(\mathcal{S}) \theta^{\mathcal{S}}(\mathcal{B})-\sum_{j \in \mathcal{N}} \theta^{\{j\}}(\mathcal{B}) .
\end{aligned}
$$

Furthermore, Lemma 1, with the positive values $\alpha(\mathcal{S}) \geq 0$, guarantees that

$$
\sum_{S \in 2^{\mathcal{N}}} \alpha(\mathcal{S}) \theta^{\mathcal{S}}(\mathcal{B}) \leq \theta^{\sum_{S \in 2 \mathcal{N}} \alpha(\mathcal{S}) \mathcal{S}}(\mathcal{B})
$$

And, by definition of the coalition summation in (A.1), the optimal value indexed by coalition superscripts can be reformulated as

$$
\begin{aligned}
\theta^{\sum_{S \in 2^{\mathcal{N}}} \alpha(\mathcal{S}) \mathcal{S}}(\mathcal{B}) & =\theta^{\sum_{S \in 2^{\mathcal{N}}} \alpha(\mathcal{S}) \sum_{j \in \mathcal{S}}\{j\}}(\mathcal{B})=\theta^{\sum_{j \in \mathcal{N}}\left(\sum_{S \in 2^{\mathcal{N}}} \alpha(\mathcal{S}) \delta_{j}^{\mathcal{S}}\right)\{j\}}(\mathcal{B})=\theta^{\sum_{j \in \mathcal{N}}\{j\}}(\mathcal{B}) \\
& =\theta^{\mathcal{N}}(\mathcal{B}) .
\end{aligned}
$$

By replacing (C.7) and (C.8) in the right-hand side of (C.6), we get

$$
\sum_{S \in 2^{\mathcal{N}}} \alpha(\mathcal{S}) v^{\mathcal{S}} \leq \theta^{\mathcal{N}}(\mathcal{B})-\sum_{j \in \mathcal{N}} \theta^{\{j\}}(\mathcal{B})=v^{\mathcal{N}} \theta^{\mathcal{N}}(\mathcal{B})
$$

This inequality shows that the cooperative game considered in this work is balanced, and has a non-empty core. 


\section{Appendix C.3. Proof of Proposition 3: bidder-optimality of core-selecting mechanisms}

Let us consider a core-selecting mechanism that, for the submitted bids $\mathcal{B}$, selects the outcomes $X^{\mathcal{N}^{*}}(\mathcal{B}), \pi\left(X^{\mathcal{N}^{*}}(\mathcal{B})\right)$ of the energy management problem $(7)$, such that the allocation mechanism is in the revealed core $\mathcal{C}\left(\mathcal{N}, v^{\mathcal{N}}(\mathcal{B})\right)$. Let us show that this allocation mechanism is Pareto efficient for the agents. This property directly ensues from the efficient allocation property of the revealed core.

We assume that there exists one other allocation in the revealed core $\hat{\pi}(\mathcal{B}) \in \mathcal{C}\left(\mathcal{N}, v^{\mathcal{N}}(\mathcal{B})\right)$ that dominates $\pi\left(X^{\mathcal{N}^{*}}(\mathcal{B})\right)$, i.e., $\hat{\pi}_{j}(\mathcal{B}) \geq \pi_{j}\left(X^{\mathcal{N}^{*}}(\mathcal{B})\right), \quad \forall j \in \mathcal{N}$ and the inequality is strict for at least one prosumer $j \in \mathcal{N}$. As a result, the efficient allocation property ensures that

$$
v^{\mathcal{N}}(\mathcal{B})=\sum_{j \in \mathcal{N}} \hat{\pi}_{j}(\mathcal{B})>\sum_{j \in \mathcal{N}} \pi_{j}\left(X^{\mathcal{N}^{*}}(\mathcal{B})\right)=v^{\mathcal{N}}(\mathcal{B})
$$

As $v^{\mathcal{N}}(\mathcal{B})$ is defined as the global minimum of a convex function over a convex and compact set, the strict inequality (C.10) is impossible. This shows that there exists no such allocation $\hat{\pi}(\mathcal{B})$ in the revealed core that dominates $\pi\left(X^{\mathcal{N}^{*}}(\mathcal{B})\right)$. This shows that, by design, all core-selecting mechanisms for the cooperative game defined in (9) are bidder efficient and thus incentive-optimal.

\section{Appendix C.4. Proof of Proposition 4: group rationality of uniform pricing}

In the proposed community-based market mechanism, we demonstrate that the uniform pricing payment rule is a core-selecting mechanism under the assumption of perfect competition.

\section{Appendix C.4.1. Non-cooperative equilibrium under perfect competition assumption}

Under the assumption of perfect competition, the uniform prices payment rule has been shown to be dominant-strategy incentive compatible and efficient ${ }^{3}$. Hence, truthful bidding, i.e., $\mathcal{B}=\tilde{\mathcal{B}}$, is a dominant strategy for all agents $j \in \mathcal{N}$. Additionally, all optimal solutions of the energy procurement problem (7) coincide with and efficient Nash equilibriums of the utility maximization problems of the individual agents.

The non-cooperative equilibrium problem, where the prosumers in a coalition $j \in \mathcal{S} \in 2^{\mathcal{N}}$ try to maximize their individual utilities $u_{j}\left(X_{j}(\mathcal{B}), \lambda\left(\mathcal{B}, \mathcal{B}_{j}\right)\right)$, is denoted by $E q{ }^{\mathcal{S}}$. Under perfect competition assumption, the utility maximization problem of an individual agent in the grand coalition $j \in \mathcal{N}$ in this equilibrium problem $E q{ }^{\mathcal{S}}$ can be formulated as follows:

$$
\max _{X_{j} \in \mathcal{K}_{j}^{\mathcal{S}}\left(\mathcal{B}_{j}\right)} u_{j}\left(X_{j}, \lambda, \mathcal{B}_{j}\right)
$$

where the feasible space is defined as

$$
\mathcal{K}_{j}^{\mathcal{S}}=\left\{X_{j} \in \mathbb{R}_{K_{2}} \mid Q_{j}^{(2)}\left(X_{j} \circ Q_{j}^{(1)}\right) \leq Q_{j}^{0^{\mathcal{S}}}\right\}
$$

Note that the expression of the feasible space $\mathcal{K}_{j}^{\mathcal{S}}\left(\mathcal{B}_{j}\right)$ of each prosumer $j \in \mathcal{N}$, indexed by the coalition $\mathcal{S}$, ensures that any feasible decision variable $X_{j}^{\mathcal{S}} \in \mathcal{K}_{j}\left(\mathcal{B}_{j}\right)$ is fixed to zero if $j \notin \mathcal{S}$. In addition, the linking constraints $(4 \mathrm{a})$ and (4b) are added to this equilibrium problem to ensure

\footnotetext{
${ }^{3}$ The proofs of these properties rely on the strong duality and complementarity theorems of the energy management problem.
} 
balance of heat and electricity exchanges within the coalition $\mathcal{S} \in 2^{\mathcal{N}}$. The uniform prices $\lambda^{\mathrm{E}} \in \mathbb{R}^{T}$ and $\lambda^{\mathrm{H}} \in \mathbb{R}^{T}$ are derived as dual variables of these balance equations at equilibrium.

The objective of the utility maximization problem (C.11) of each prosumer depends on the uniform prices $\lambda=\left[\lambda^{\mathrm{E}}, \lambda^{\mathrm{H}}\right] \in \mathbb{R}^{2 T}$. Under the assumption of perfect competition, each prosumer models these prices are independent from its decisions, i.e., as fixed parameters in (C.11). It results from this assumption, that the KKT conditions of the equilibrium problem $E q \cdot{ }^{\mathcal{S}}$ are identical to the KKT conditions of the energy management problem (7) for a coalition $\mathcal{S} \in 2^{\mathcal{N}}$, which are detailed in Appendix B.3. As these KKT conditions are necessary and sufficient, any optimal solution to the energy management problem (7) for a coalition $\mathcal{S} \in 2^{\mathcal{N}}$ coincides with an efficient Nash equilibrium of the equilibrium problem $E q \cdot{ }^{\mathcal{S}}$, and vice versa.

\section{Appendix C.4.2. Efficient allocation}

It has been shown that the uniform pricing payment rule $\mathcal{P}^{\lambda}$ is budget-balanced for any revealed preferences $\mathcal{B} \in \mathbb{R}^{K_{1} \times N}$. In other words, it defines an efficient allocation mechanism $\pi^{\lambda}$, such that $\pi_{j}^{\lambda}\left(X_{j}^{\mathcal{N}^{*}}(\mathcal{B}), \lambda^{\mathcal{N}^{*}}(\mathcal{B})\right)=\mathcal{P}_{j}^{\lambda}\left(X_{j}^{\mathcal{N}^{*}}(\mathcal{B}), \lambda^{\mathcal{N}^{*}}(\mathcal{B})\right)+\mathcal{F}_{j}\left(X_{j}^{\mathcal{N}^{*}}(\mathcal{B}), Q_{j}^{\mathrm{c}}\right)-\theta^{\{j\}}(\mathcal{B}), \quad \forall j \in \mathcal{N}$.

\section{Appendix C.4.3. Group rationality}

Let us consider a coalition of prosumers $\mathcal{S} \in 2^{\mathcal{N}}$, and show that the excess of the coalition $\epsilon_{\mathcal{S}}\left(\pi\left(X^{\mathcal{N}^{*}}(\mathcal{B})\right)\right)$ is negative.

By definition of the payment rule and allocation mechanism in (10), the excess $\epsilon_{\mathcal{S}}\left(\pi\left(X^{\mathcal{N}^{*}}(\mathcal{B})\right)\right)$ of the coalition can be reformulated as

$$
\begin{aligned}
\epsilon_{\mathcal{S}}\left(\pi^{\lambda}\left(X^{\mathcal{N}^{*}}(\mathcal{B}), \lambda^{\mathcal{N}^{*}}(\mathcal{B})\right)\right) & =v^{\mathcal{S}}(\mathcal{B})-\sum_{j \in \mathcal{S}}\left[\mathcal{P}_{j}^{\lambda}\left(X_{j}^{\mathcal{N}^{*}}(\mathcal{B}), \lambda^{\mathcal{N}^{*}}(\mathcal{B})\right)+\mathcal{F}_{j}\left(X_{j}^{\mathcal{N}^{*}}, Q_{j}^{\mathrm{c}}\right)-\theta^{\{j\}}(\mathcal{B})\right] \\
& =\theta^{\mathcal{S}}(\mathcal{B})-\sum_{j \in \mathcal{S}}\left[\mathcal{P}_{j}^{\lambda}\left(X_{j}^{\mathcal{N}^{*}}(\mathcal{B}), \lambda^{\mathcal{N}^{*}}(\mathcal{B})\right)+\mathcal{F}_{j}\left(X_{j}^{\mathcal{N}^{*}}, Q_{j}^{\mathrm{c}}\right)\right] \\
& =\sum_{j \in \mathcal{S}}\left[u_{j}\left(X_{j}^{\mathcal{S}^{*}}(\mathcal{B}), \lambda^{\mathcal{S}^{*}}(\mathcal{B}), \mathcal{B}\right)-u_{j}\left(X_{j}^{\mathcal{N}^{*}}(\mathcal{B}), \lambda^{\mathcal{N}^{*}}(\mathcal{B}), \mathcal{B}\right)\right] .
\end{aligned}
$$

Outcomes of the mechanism in the grand-coalition $\mathcal{N}$ and the coalition $\mathcal{S} \in 2^{\mathcal{N}}$ are denoted by $\left(X^{\mathcal{N}^{*}}(\mathcal{B}), \lambda^{\mathcal{N}^{*}}(\mathcal{B})\right)$ and $\left(X^{\mathcal{S}^{*}}(\mathcal{B}), \lambda^{\mathcal{S}^{*}}(\mathcal{B})\right)$, respectively. As discussed above, these optimal solutions of the convex energy management problem (7) and their associated dual solutions are also Nash equilibriums of the equilibrium problems $E q \cdot^{\mathcal{N}}$ and $E q{ }^{\mathcal{S}}$, respectively. By definition of a Nash equilibrium, and under the assumption of perfect competition, it follows that

$$
u_{j}\left(X_{j}^{\mathcal{N}^{*}}(\mathcal{B}), \lambda^{\mathcal{N}^{*}}(\mathcal{B})\right)=\max _{X_{j} \in \mathcal{K}_{j}^{\mathcal{N}}} u_{j}\left(X_{j}, \lambda^{\mathcal{N}^{*}}(\mathcal{B})\right), \quad \forall j \in \mathcal{N}
$$

Furthermore, for any prosumer $j \in \mathcal{S}, X_{j}^{\mathcal{S}^{*}} \in \mathcal{K}_{j}^{\mathcal{N}}$, which implies that

$$
u_{j}\left(X_{j}^{\mathcal{N}^{*}}(\mathcal{B}), \lambda^{\mathcal{N}^{*}}(\mathcal{B})\right) \geq u_{j}\left(X_{j}^{\mathcal{S}^{*}}(\mathcal{B}), \lambda^{\mathcal{N}^{*}}(\mathcal{B})\right), \quad \forall j \in \mathcal{S} .
$$

By replacing these inequalities in the expression of the excess of the coalition in (C.13), it results that this excess is negative. Hence, we have proved that under the assumption of perfect competition, the allocation mechanism $\pi^{\lambda}$ respects the group rationality. Along with the efficient allocation property, it results that this is a core-selecting mechanism, under the assumption of perfect competition 


\section{Appendix D. Case study setup}

This section details the setup of the case study used in Section 5 .

Table D.6: Preferences: Technical parameters of the assets and building of each prosumer.

\begin{tabular}{|c|c|c|c|c|c|}
\hline & & Christos & Anna & Vladimir & Andrea \\
\hline$T^{\text {set }}$ & $\left({ }^{\circ} \mathrm{C}\right)$ & 20 & 20 & 20 & 20 \\
\hline $\bar{u}^{\mathrm{up}}$ & $\left({ }^{\circ} \mathrm{C}\right)$ & 1 & 1 & 0 & 1 \\
\hline $\bar{u}^{\text {down }}$ & $\left({ }^{\circ} \mathrm{C}\right)$ & 1 & 1 & 0 & 1 \\
\hline$c^{\mathrm{up}}$ & $\left(€ /{ }^{\circ} \mathrm{C}\right)$ & 0.001 & 0.001 & - & 0.001 \\
\hline$c^{\text {down }}$ & $\left(€ /{ }^{\circ} \mathrm{C}\right)$ & 0.01 & 0.01 & - & 0.01 \\
\hline $\bar{L}^{\mathrm{HP}}$ & $(\mathrm{kW})$ & - & 10 & - & - \\
\hline$C O P^{\mathrm{HP}}$ & - & 2.5 & - & - & - \\
\hline$\overline{e n}^{\mathrm{E}} / e n^{\mathrm{H}, 0}$ & $(\mathrm{kWh})$ & $5 / 2.5$ & - & - & - \\
\hline$\overline{c h}^{\mathrm{E}} / \overline{d i s}^{\mathrm{E}}$ & $(\mathrm{kW})$ & $2 / 2$ & - & - & - \\
\hline$\rho^{d i s^{\mathrm{E}}} / \rho^{c h^{\mathrm{E}}}$ & - & $1.1 / 0.9$ & - & - & - \\
\hline$\overline{e n}^{\mathrm{H}} / e n^{\mathrm{H}, 0}$ & (kWh) & - & 2.5 & - & - \\
\hline$\overline{c h}^{\mathrm{H}} / \overline{d i s}^{\mathrm{H}}$ & $(\mathrm{kW})$ & - & $2 / 2$ & - & - \\
\hline$\rho^{d i s^{\mathrm{H}}} / \rho^{c h^{\mathrm{H}}}$ & - & - & $1.1 / 0.9$ & - & - \\
\hline $\bar{f}^{\mathrm{E}}$ & $(\mathrm{kW})$ & 5 & 5 & 5 & 5 \\
\hline $\bar{f}^{\mathrm{H}}$ & $(\mathrm{kW})$ & 5 & 5 & 5 & 5 \\
\hline $\bar{b}^{\mathrm{E}}, \bar{s}^{\mathrm{E}}$ & $(\mathrm{kW})$ & 5 & 5 & 5 & 5 \\
\hline $\bar{b}^{\mathrm{H}}, \bar{s}^{\mathrm{H}}$ & $(\mathrm{kW})$ & 5 & 5 & 5 & 5 \\
\hline$\alpha^{1}$ & - & 1.0062 & 1.0062 & 1.0062 & 1.0062 \\
\hline$\alpha^{2}$ & - & 0.0036 & 0.0036 & 0.0036 & 0.0036 \\
\hline$\alpha^{3}$ & $\left(0^{-5}\right)$ & 6.17 & 6.17 & 6.17 & 6.17 \\
\hline$\beta^{1}$ & - & 0.0011 & 0.0011 & 0.0011 & 0.0011 \\
\hline$\beta^{2}$ & - & 1.0021 & 1.0021 & 1.0021 & 1.0021 \\
\hline
\end{tabular}

Furthermore, the outdoor temperatures $T^{\mathrm{e}}$ and dynamic technical parameters $\alpha^{0}$ and $\beta^{0}$ of the buildings are assumed identical for all buildings in the energy community, and represented in Figure D.3 over all hours of the day.

For the sake of simplicity, solely space heating loads are considered flexible. Table D.7 illustrates the temperature preferences of each prosumer and the cost for deviating from the temperature setpoint, which are considered constant over all hours of the day.

Table D.7: Preferences: Temperature preferences and utilities.

\begin{tabular}{lccccc} 
& & Christos & Anna & Vladimir & Andrea \\
\hline$T^{\text {set }}$ & $\left({ }^{\circ} \mathrm{C}\right)$ & 20 & 20 & 20 & 20 \\
$\bar{u}^{\text {up }}$ & $\left({ }^{\circ} \mathrm{C}\right)$ & 1 & 1 & 0 & 1 \\
$\bar{u}^{\text {down }}$ & $\left({ }^{\circ} \mathrm{C}\right)$ & 1 & 1 & 0 & 1 \\
$c^{\text {up }}$ & $\left(€ /{ }^{\circ} \mathrm{C}\right)$ & 0.001 & 0.001 & - & 0.001 \\
$c^{\text {down }}$ & $\left(€ /{ }^{\circ} \mathrm{C}\right)$ & 0.01 & 0.01 & - & 0.01 \\
\hline
\end{tabular}




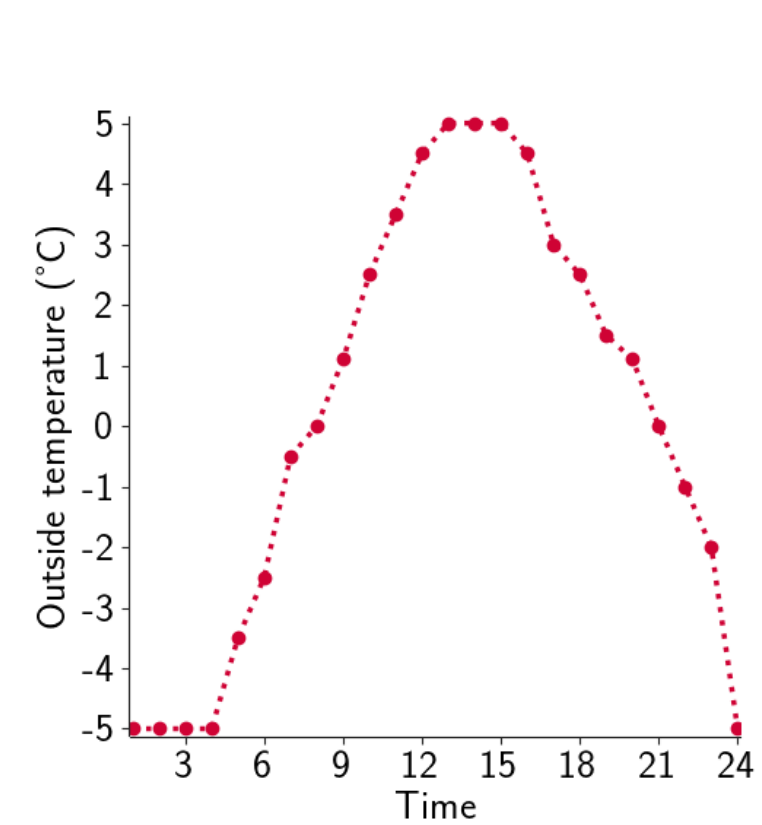

(a)

\section{$\cdots \times \cdot \alpha^{0}\left(10^{-2}\right) \quad \cdots \times \cdots \quad \beta^{0}\left(10^{-3}\right)$}

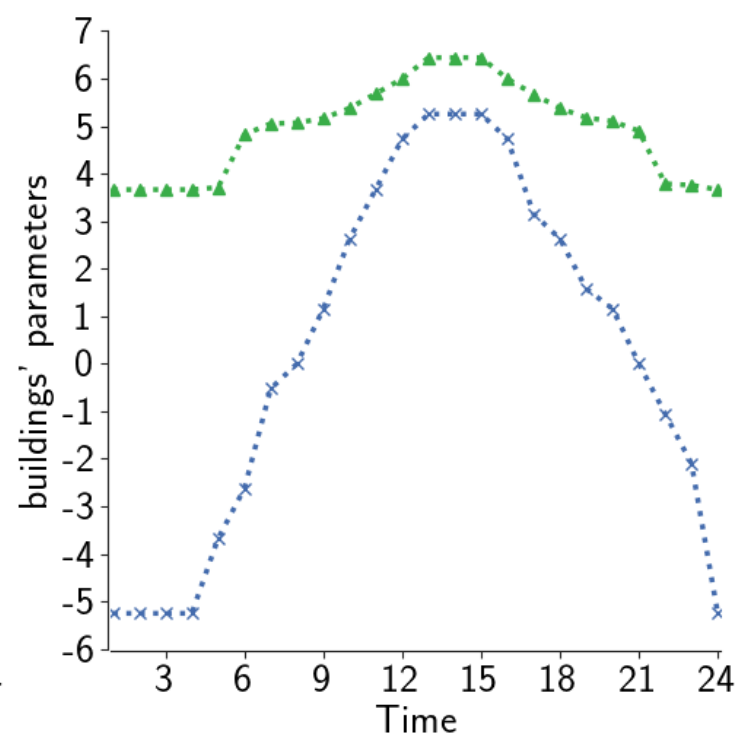

(b)

Figure D.3: (a) Outdoor temperatures $T^{\mathrm{e}}$, and (b) technical parameters $\alpha^{0}$ and $\beta^{0}$ of the buildings in the energy community.

Additionally, Figures D.4(a) and D.4(b) illustrate the inflexible electricity and hot water loads of each prosumer over all hours of the day. The electricity production from solar panels belonging to Christos is considered perfectly known, and illustrated in Figure D.4(c) over all hours of the day.

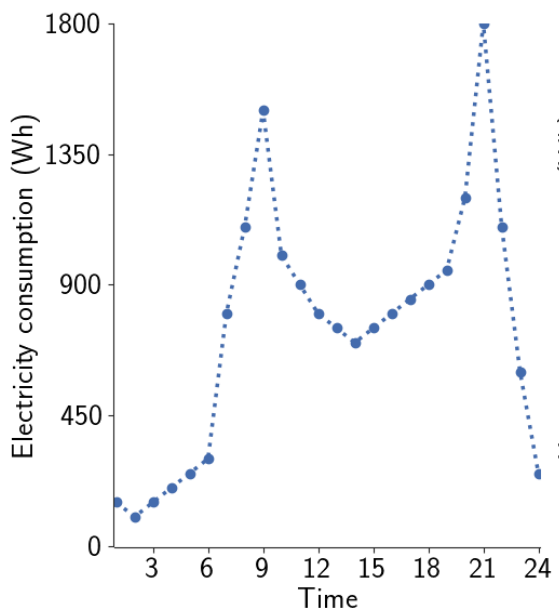

(b)

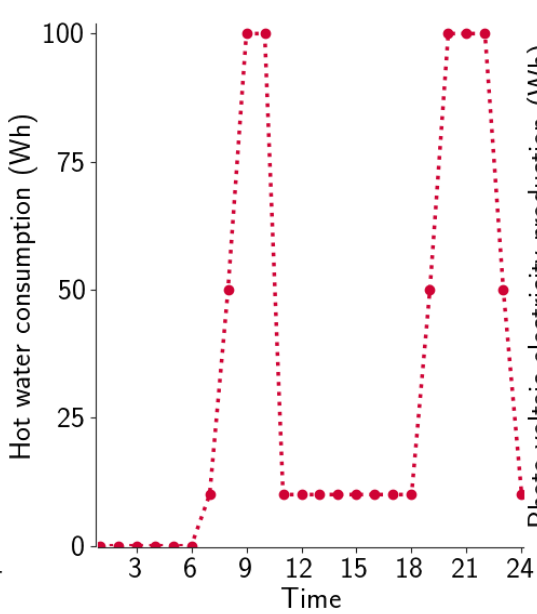

(c)

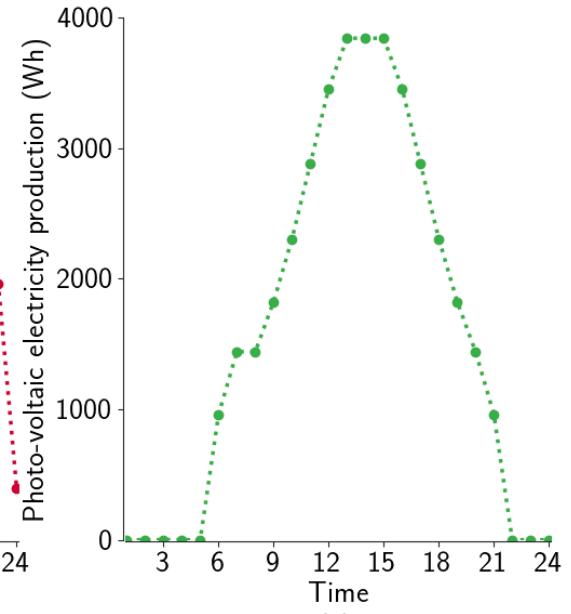

(a)

Figure D.4: Inflexible (a) electricity from electrical appliances $L^{\mathrm{E}}$, and (b) hot water load $L^{\mathrm{HW}}$ of each prosumer in the energy community, and (c) photo-voltaic electricity production $\overline{P h i}^{\text {ENR }}$ from Christos.

Finally, prosumers can import or export electricity and heat from the grid at an hourly tariff, 
as illustrated in Figures D.5(a) and D.5(b).

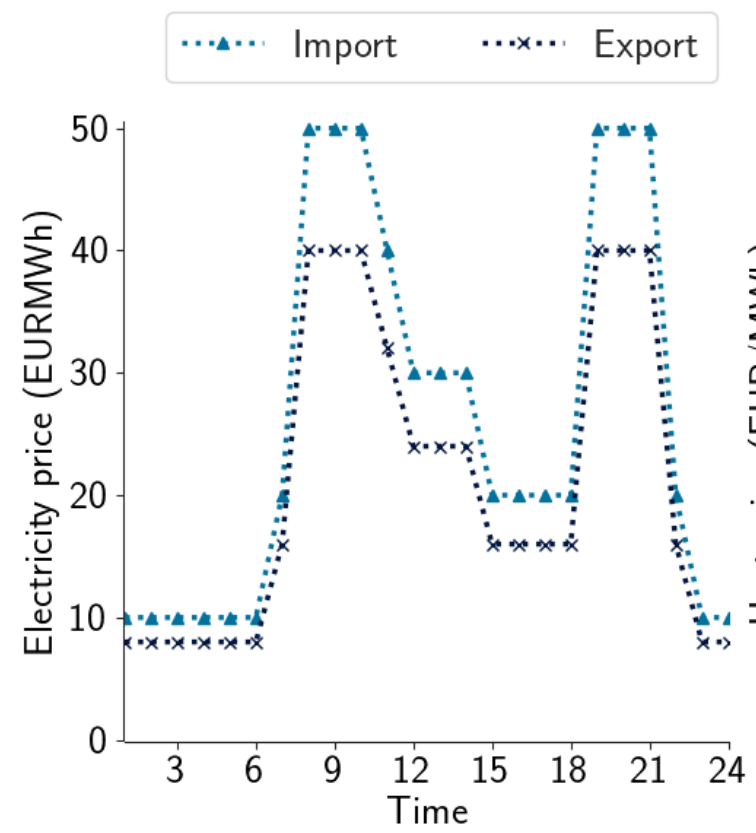

(a)

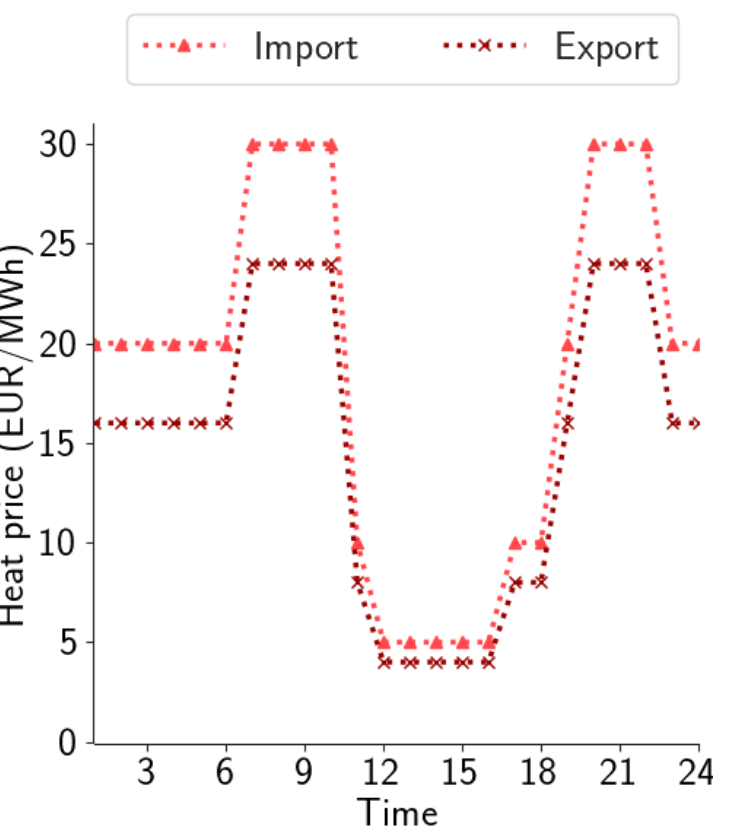

(b)

Figure D.5: Hourly tariffs for exchanges with the (a) electricity and (b) heat grids.

\section{References}

Alahäivälä, A., Corbishley, J., Ekström, J., Jokisalo, J., \& Lehtonen, M. (2017). A control framework for the utilization of heating load flexibility in a day-ahead market. Electric Power Systems Research, 145, 44-54.

Alam, M., Ramchurn, S. D., \& Rogers, A. (2013). Cooperative energy exchange for the efficient use of energy and resources in remote communities. In Proceedings of the 2013 International Conference on Autonomous Agents and Multi-Agent Systems (pp. 731-738). International Foundation for Autonomous Agents and Multiagent Systems.

Alimohammadisagvand, B., Jokisalo, J., Kilpeläinen, S., Ali, M., \& Sirén, K. (2016). Cost-optimal thermal energy storage system for a residential building with heat pump heating and demand response control. Applied Energy, $174,275-287$.

Allcott, H., \& Mullainathan, S. (2010). Behavior and energy policy. Science, 327, 1204-1205.

Angeletos, G.-M., Hellwig, C., \& Pavan, A. (2007). Dynamic global games of regime change: Learning, multiplicity, and the timing of attacks. Econometrica, 75, 711-756.

Arthur, W. B. (1994). Inductive reasoning and bounded rationality. The American Economic Review, 84, $406-411$. Ausubel, L. M., \& Milgrom, P. (2006). The lovely but lonely Vickrey auction. Combinatorial Auctions, 17, 22-26.

Bellet, A., Guerraoui, R., Taziki, M., \& Tommasi, M. (2017). Fast and Differentially Private Algorithms for Decentralized Collaborative Machine Learning. Ph.D. thesis INRIA Lille.

Bernheim, B. D., Peleg, B., \& Whinston, M. D. (1987). Coalition-proof Nash equilibria I. concepts. Journal of Economic Theory, 42, 1-12.

Bernheim, B. D., \& Whinston, M. D. (1987). Coalition-proof Nash equilibria II. applications. Journal of Economic Theory, 42, 13-29.

Bobo, L. A., Delikaraoglou, S., Vespermann, N., Kazempour, J., \& Pinson, P. (2018). Offering strategy of a flexibility aggregator in a balancing market using asymmetric block offers. In Power Systems Computation Conference $(P S C C)$. IEEE.

Bouffard, F., \& Kirschen, D. S. (2008). Centralised and distributed electricity systems. Energy Policy, 36, 4504-4508.

Brahman, F., Honarmand, M., \& Jadid, S. (2015). Optimal electrical and thermal energy management of a residential energy hub, integrating demand response and energy storage system. Energy and Buildings, 90, 65-75. 
Brange, L., Englund, J., \& Lauenburg, P. (2016). Prosumers in district heating networks-a swedish case study. Applied Energy, 164, 492-500.

Callaway, D. S., \& Hiskens, I. A. (2011). Achieving controllability of electric loads. Proceedings of the IEEE, 99, 184-199.

Cavallo, R. (2006). Optimal decision-making with minimal waste: Strategy-proof redistribution of VCG payments. In Proceedings of the 5th International Joint Conference on Autonomous Agents and Multiagent Systems (pp. 882-889).

Chu, S., Cui, Y., \& Liu, N. (2017). The path towards sustainable energy. Nature Materials, $16,16$.

Chu, S., \& Majumdar, A. (2012). Opportunities and challenges for a sustainable energy future. Nature, 488, $294-303$.

Clarke, E. H. (1971). Multipart pricing of public goods. Public Choice, 11, 17-33.

Da Silva, P. G., Karnouskos, S., \& Ilic, D. (2012). A survey towards understanding residential prosumers in smart grid neighbourhoods. In 2012 3rd IEEE PES Innovative Smart Grid Technologies Europe (ISGT Europe) (pp. 1-8). IEEE.

Darivianakis, G., Georghiou, A., Eichler, A., Smith, R. S., \& Lygeros, J. (2017a). Scalability through decentralization: A robust control approach for the energy management of a building community. IFAC-PapersOnLine, 50, 1431414319.

Darivianakis, G., Georghiou, A., \& Lygeros, J. (2018). Decentralized decision making for networks of uncertain systems. In arXiv preprint arXiv:1803.07660.

Darivianakis, G., Georghiou, A., Smith, R. S., \& Lygeros, J. (2015). A stochastic optimization approach to cooperative building energy management via an energy hub. In 2015 IEEE 54th Annual Conference on Decision and Control (CDC) (pp. 7814-7819). IEEE.

Darivianakis, G., Georghiou, A., Smith, R. S., \& Lygeros, J. (2017b). The power of diversity: Data-driven robust predictive control for energy-efficient buildings and districts. IEEE Transactions on Control Systems Technology, 27, $132-145$.

Day, R., \& Milgrom, P. (2008). Core-selecting package auctions. International Journal of Game Theory, 36, $393-407$.

De Paepe, M., D'Herdt, P., \& Mertens, D. (2006). Micro-CHP systems for residential applications. Energy Conversion and Management, 4\%, 3435-3446.

Dominković, D., Gianniou, P., Münster, M., Heller, A., \& Rode, C. (2018). Utilizing thermal building mass for storage in district heating systems: Combined building level simulations and system level optimization. Energy, 153, 949-966.

Dwork, C. (2008). Differential privacy: A survey of results. In International Conference on Theory and Applications of Models of Computation (pp. 1-19). Springer.

Dwork, C., \& Roth, A. (2014). The algorithmic foundations of differential privacy. Foundations and Trends in Theoretical Computer Science, 9, 211-407.

Energylab Nordhavn project (2019). Reports and downloads. URL: http://www.energylabnordhavn.com/ reports-and-downloads.html.

Exizidis, L., Kazempour, J., Papakonstantinou, A., Pinson, P., De Greve, Z., \& Vallee, F. (2019). Incentivecompatibility in a two-stage stochastic electricity market with high wind power penetration. IEEE Transactions on Power Systems, 34, 2846-2858.

Forges, F., Heifetz, A., \& Minelli, E. (2001). Incentive compatible core and competitive equilibria in differential information economies. Economic Theory, 18, 349-365.

Forges, F., \& Minelli, E. (2001). A note on the incentive compatible core. Journal of Economic Theory, 98, $179-188$.

Gangale, F., Mengolini, A., \& Onyeji, I. (2013). Consumer engagement: An insight from smart grid projects in europe. Energy Policy, 60, 621-628.

Geidl, M., Koeppel, G., Favre-Perrod, P., Klockl, B., Andersson, G., \& Frohlich, K. (2007). Energy hubs for the future. IEEE Power and Energy Magazine, 5, 24-30.

Gigerenzer, G., \& Selten, R. (2002). Bounded Rationality: The Adaptive Toolbox. MIT Press.

Good, N., Ellis, K. A., \& Mancarella, P. (2017). Review and classification of barriers and enablers of demand response in the smart grid. Renewable and Sustainable Energy Reviews, 72, 57-72.

Good, N., \& Mancarella, P. (2017). Flexibility in multi-energy communities with electrical and thermal storage: A stochastic, robust approach for multi-service demand response. IEEE Transactions on Smart Grid, 10, 503-513.

Goulden, M., Bedwell, B., Rennick-Egglestone, S., Rodden, T., \& Spence, A. (2014). Smart grids, smart users? the role of the user in demand side management. Energy Research \& Social Science, 2, 21-29.

Green, J. R., \& Laffont, J.-J. (1979). Incentives in Public Decision Making. North-Holland.

Groves, T. (1973). Incentives in teams. Econometrica, (pp. 617-631).

Guajardo, M., \& Jörnsten, K. (2015). Common mistakes in computing the nucleolus. European Journal of Operational 
Research, 241, 931-935.

Gyamfi, S., Krumdieck, S., \& Urmee, T. (2013). Residential peak electricity demand response-highlights of some behavioural issues. Renewable and Sustainable Energy Reviews, 25, 71-77.

Han, L., Morstyn, T., \& McCulloch, M. (2018). Incentivizing prosumer coalitions with energy management using cooperative game theory. IEEE Transactions on Power Systems, 34, 303-313.

Haring, T. W., Mathieu, J. L., \& Andersson, G. (2016). Comparing centralized and decentralized contract design enabling direct load control for reserves. IEEE Transactions on Power Systems, 31, 2044-2054.

Heinrichs, H. (2013). Sharing economy: A potential new pathway to sustainability. GAIA-Ecological Perspectives for Science and Society, 22, 228-231.

Hobbs, B. F., Helman, U., \& Bunn, D. W. (2004). Complementarity-based equilibrium modeling for electric power markets. In Modeling Prices in Competitive Electricity Markets (pp. 69-94). Wiley Series in Financial Economics.

Hobbs, B. F., Rothkopf, M. H., Hyde, L. C., \& O'Neill, R. P. (2000). Evaluation of a truthful revelation auction in the context of energy markets with nonconcave benefits. Journal of Regulatory Economics, 18, 5-32.

Houwing, M., Negenborn, R. R., \& De Schutter, B. (2011). Demand response with micro-CHP systems. Proceedings of the IEEE, 99, 200-213.

Huang, Z., Mitra, S., \& Vaidya, N. (2015). Differentially private distributed optimization. In Proceedings of the 2015 International Conference on Distributed Computing and Networking.

Hurwicz, L. (1972). On informationally decentralized systems. In Decision and Organization: A Volume in Honor of J. Marschak. North-Holland.

Hurwicz, L. (1973). The design of mechanisms for resource allocation. The American Economic Review, 63, 1-30.

Hurwicz, L., \& Reiter, S. (2006). Designing Economic Mechanisms. Cambridge University Press.

Jaffe, A. B., \& Stavins, R. N. (1994). The energy-efficiency gap what does it mean? Energy Policy, 22, 804-810.

Joskow, P., \& Kahn, E. (2001). A quantitative analysis of pricing behavior in California's wholesale electricity market during summer 2000. In Power Engineering Society Summer Meeting (pp. 392-394). IEEE volume 1.

Kahneman, D., \& Tversky, A. (2013). Prospect theory: An analysis of decision under risk. In Handbook of the Fundamentals of Financial Decision Making: Part I (pp. 99-127). World Scientific.

Kalathil, D., Wu, C., Poolla, K., \& Varaiya, P. (2017). The sharing economy for the electricity storage. IEEE Transactions on Smart Grid, 10, 556-565.

Kalkbrenner, B. J., \& Roosen, J. (2016). Citizens' willingness to participate in local renewable energy projects: The role of community and trust in germany. Energy Research \&6 Social Science, 13, 60-70.

Karaca, O., \& Kamgarpour, M. (2017). Game theoretic analysis of electricity market auction mechanisms. In 2017 IEEE 56th Annual Conference on Decision and Control (CDC) (pp. 6211-6216). IEEE.

Karaca, O., Sessa, P. G., Walton, N., \& Kamgarpour, M. (2019). Designing coalition-proof reverse auctions over continuous goods. IEEE Transactions on Automatic Control, 64, 4803-4810.

Kazempour, J., Pinson, P., \& Hobbs, B. F. (2018). A stochastic market design with revenue adequacy and cost recovery by scenario: Benefits and costs. IEEE Transactions on Power Systems, 33, 3531-3545.

Khazaei, H., \& Zhao, Y. (2019). Indirect mechanism design for efficient and stable renewable energy aggregation. IEEE Transactions on Power Systems, 34, 1033-1042.

Kılkıs, S. (2015). Exergy transition planning for net-zero districts. Energy, 92, 515-531.

Kirschen, D. S. (2003). Demand-side view of electricity markets. IEEE Transactions on power systems, 18, 520-527.

Klein, L., Kwak, J.-y., Kavulya, G., Jazizadeh, F., Becerik-Gerber, B., Varakantham, P., \& Tambe, M. (2012). Coordinating occupant behavior for building energy and comfort management using multi-agent systems. Automation in Construction, 22, 525-536.

Klein, S. J., \& Coffey, S. (2016). Building a sustainable energy future, one community at a time. Renewable and Sustainable Energy Reviews, 60, 867-880.

Koch, E., \& Piette, M. A. (2009). Direct versus facility centric load control for automated demand response. Technical Report Lawrence Berkeley National Lab.(LBNL), Berkeley, CA (United States).

Lampropoulos, I., Vanalme, G. M., \& Kling, W. L. (2010). A methodology for modeling the behavior of electricity prosumers within the smart grid. In 2010 IEEE PES Innovative Smart Grid Technologies Conference Europe (ISGT Europe) (pp. 1-8). IEEE.

Lesage-Landry, A., \& Taylor, J. A. (2017). Learning to shift thermostatically controlled loads. In Proceedings of the 50th Hawaii International Conference on System Sciences.

Liu, Y., Holzer, J. T., \& Ferris, M. C. (2015). Extending the bidding format to promote demand response. Energy Policy, 86, 82-92.

Lund, H. (2005). Large-scale integration of wind power into different energy systems. Energy, 30, 2402-2412.

Lund, H. (2007). Renewable energy strategies for sustainable development. Energy, 32, 912-919. 
Lund, H., Möller, B., Mathiesen, B. V., \& Dyrelund, A. (2010). The role of district heating in future renewable energy systems. Energy, 35, 1381-1390.

Lund, H., Werner, S., Wiltshire, R., Svendsen, S., Thorsen, J. E., Hvelplund, F., \& Mathiesen, B. V. (2014). 4 th generation district heating $(4 \mathrm{GDH})$ : Integrating smart thermal grids into future sustainable energy systems. Energy, 68, 1-11.

Lund, P. D., Lindgren, J., Mikkola, J., \& Salpakari, J. (2015a). Review of energy system flexibility measures to enable high levels of variable renewable electricity. Renewable and Sustainable Energy Reviews, 45, 785-807.

Lund, P. D., Mikkola, J., \& Ypyä, J. (2015b). Smart energy system design for large clean power schemes in urban areas. Journal of Cleaner Production, 103, 437-445.

Maskin, E. (1999). Nash equilibrium and welfare optimality. The Review of Economic Studies, 66, 23-38.

McSherry, F., \& Talwar, K. (2007). Mechanism design via differential privacy. In 48th Annual IEEE Symposium on Foundations of Computer Science (FOCS'07) (pp. 94-103). IEEE.

Medina, J., Muller, N., \& Roytelman, I. (2010). Demand response and distribution grid operations: Opportunities and challenges. IEEE Transactions on Smart Grid, 1, 193-198.

Meibom, P., Hilger, K. B., Madsen, H., \& Vinther, D. (2013). Energy comes together in Denmark: The key to a future fossil-free Danish power system. IEEE Power Energy Magazine, 11, 46-55.

Meibom, P., Kiviluoma, J., Barth, R., Brand, H., Weber, C., \& Larsen, H. V. (2007). Value of electric heat boilers and heat pumps for wind power integration. Wind Energy: An International Journal for Progress and Applications in Wind Power Conversion Technology, 10, 321-337.

Ming, H., \& Xie, L. (2014). Analysis of coupon incentive-based demand response with bounded consumer rationality. In North American Power Symposium (NAPS) (pp. 1-6). IEEE.

Mitridati, L., \& Pinson, P. (2016). Optimal coupling of heat and electricity systems: A stochastic hierarchical approach. In 2016 International Conference on Probabilistic Methods Applied to Power Systems (PMAPS) (pp. 1-6). IEEE.

Morales, J. M., Conejo, A. J., Madsen, H., Pinson, P., \& Zugno, M. (2013). Integrating Renewables in Electricity Markets: Operational Problems. Springer Science \& Business Media.

Moret, F., Baroche, T., Sorin, E., \& Pinson, P. (2018). Negotiation algorithms for peer-to-peer electricity markets: Computational properties. In 2018 Power Systems Computation Conference (PSCC) (pp. 1-7). IEEE.

Moret, F., \& Pinson, P. (2019). Energy collectives: A community and fairness based approach to future electricity markets. IEEE Transactions on Power Systems, 34, 3994-4004.

Morstyn, T., Farrell, N., Darby, S. J., \& McCulloch, M. D. (2018). Using peer-to-peer energy-trading platforms to incentivize prosumers to form federated power plants. Nature Energy, 3, 94-101.

Morstyn, T., \& McCulloch, M. (2019). Multiclass energy management for peer-to-peer energy trading driven by prosumer preferences. IEEE Transactions on Power Systems, 34, 4005-4014.

Morstyn, T., Teytelboym, A., \& McCulloch, M. D. (2019). Bilateral contract networks for peer-to-peer energy trading. IEEE Transactions on Smart Grid, 10, 2026-2035.

Münster, M., Morthorst, P. E., Larsen, H. V., Bregnbæk, L., Werling, J., Lindboe, H. H., \& Ravn, H. (2012). The role of district heating in the future Danish energy system. Energy, 48, 47-55.

Myerson, R. B., \& Satterthwaite, M. A. (1983). Efficient mechanisms for bilateral trading. Journal of Economic Theory, 29, 265-281.

NERC (2010). Technical report of north american electric reliability corporation - Flexibility requirements and metrics for variable generation: Implications for system planning studies. https://www.nerc.com/files/IVGTF_ Task_1_4_Final.pdf.

Nisan, N., Roughgarden, T., Tardos, E., \& Vazirani, V. V. (2007). Algorithmic Game Theory. Cambridge University Press.

Nozari, E., Tallapragada, P., \& Cortés, J. (2018). Differentially private distributed convex optimization via functional perturbation. IEEE Transactions on Control of Network Systems, 5, 395-408.

O'Connell, N., Pinson, P., Madsen, H., \& O'Malley, M. (2016). Economic dispatch of demand response balancing through asymmetric block offers. IEEE Transactions on Power Systems, 31, 2999-3007.

O'Connell, N., Pinson, P., Madsen, H. et al. (2014). Benefits and challenges of electrical demand response: A critical review. Renewable and Sustainable Energy Reviews, 39, 686-699.

Osborne, M. J., \& Rubinstein, A. (1994). A Course in Game Theory. MIT Press.

Parag, Y., \& Sovacool, B. K. (2016). Electricity market design for the prosumer era. Nature Energy, 1, 16032.

Peters, H. (2015). Game Theory: A Multi-Leveled Approach. Springer.

Pinson, P., Mitridati, L., Ordoudis, C., \& Østergaard, J. (2017). Towards fully renewable energy systems: Experience and trends in Denmark. CSEE Journal of Power and Energy Systems, 3, 26-35. 
Rey, F., Zhang, X., Merkli, S., Agliati, V., Kamgarpour, M., \& Lygeros, J. (2019). Strengthening the group: Aggregated frequency reserve bidding with ADMM. IEEE Transactions on Smart Grid, 10, 3860-3869.

Roberts, D. J., \& Postlewaite, A. (1976). The incentives for price-taking behavior in large exchange economies. Econometrica, (pp. 115-127).

Roth, A. E. (1982). The economics of matching: Stability and incentives. Mathematics of Operations Research, 7, $617-628$.

Roth, A. E. (2000). Game theory as a tool for market design. In Game Practice: Contributions from Applied Game Theory (pp. 7-18). Springer.

Saad, W., Han, Z., Debbah, M., Hjørungnes, A., \& Basar, T. (2009). Coalitional game theory for communication networks: A tutorial. In arXiv preprint arXiv:0905.4057.

Sanstad, A. H., \& Howarth, R. B. (1994). Consumer rationality and energy efficiency. In Proceedings of the ACEEE (pp. 175-83). volume 1.

Sargent, T. J. et al. (1993). Bounded rationality in macroeconomics: The Arne Ryde memorial lectures. In OUP Catalogue. Oxford University Press.

Scarf, H. E. (1967). The core of an N person game. Econometrica, (pp. 50-69).

Schmeidler, D. (1969). The nucleolus of a characteristic function game. SIAM Journal on Applied Mathematics, 17, $1163-1170$.

Schweppe, F. C., Caramanis, M. C., Tabors, R. D., \& Bohn, R. E. (1988). Spot Pricing of Electricity. Springer Science \& Business Media.

Sessa, P. G., Walton, N., \& Kamgarpour, M. (2017). Exploring the Vickrey-Clarke-Groves mechanism for electricity markets. IFAC-PapersOnLine, 50, 189-194.

Shaikh, P. H., Nor, N. B. M., Nallagownden, P., Elamvazuthi, I., \& Ibrahim, T. (2014). A review on optimized control systems for building energy and comfort management of smart sustainable buildings. Renewable and Sustainable Energy Reviews, 34, 409-429.

Silva, C., Wollenberg, B. F., \& Zheng, C. Z. (2001). Application of mechanism design to electric power markets (republished). IEEE Transactions on Power Systems, 16, 862-869.

Simon, H. A. (1997). Models of Bounded Rationality: Empirically Grounded Economic Reason volume 3. MIT Press.

Sorin, E., Bobo, L., \& Pinson, P. (2019). Consensus-based approach to peer-to-peer electricity markets with product differentiation. IEEE Transactions on Power Systems, 34, 994-1004.

Sousa, T., Soares, T., Pinson, P., Moret, F., Baroche, T., \& Sorin, E. (2019). Peer-to-peer and community-based markets: A comprehensive review. Renewable and Sustainable Energy Reviews, 104, 367-378.

Stern, P. C. (1992). What psychology knows about energy conservation. American Psychologist, 47, 1224.

Tang, W., \& Jain, R. (2013). A Nash equilibrium need not exist in the locational marginal pricing mechanism. In arXiv preprint arXiv:1310.4282.

Tushar, W., Saha, T. K., Yuen, C., Liddell, P., Bean, R., \& Poor, H. V. (2018). Peer-to-peer energy trading with sustainable user participation: A game theoretic approach. IEEE Access, 6, 62932-62943.

Vallés, M., Reneses, J., Cossent, R., \& Frías, P. (2016). Regulatory and market barriers to the realization of demand response in electricity distribution networks: A European perspective. Electric Power Systems Research, 140, $689-698$.

Vanhaesebrouck, P., Bellet, A., \& Tommasi, M. (2017). Decentralized collaborative learning of personalized models over networks. In International Conference on Artificial Intelligence and Statistics (AISTATS).

Vickrey, W. (1961). Counterspeculation, auctions, and competitive sealed tenders. The Journal of Finance, 16, $8-37$.

Vulkan, N., Roth, A. E., \& Neeman, Z. (2013). The Handbook of Market Design. OUP Oxford.

Wang, H., Good, N., Ceseña, E. M., \& Mancarella, P. (2018). Co-optimization of a multi-energy microgrid considering multiple services. In 2018 Power Systems Computation Conference (PSCC) (pp. 1-7). IEEE.

Wang, H., Good, N., \& Mancarella, P. (2017a). Modeling and valuing multi-energy flexibility from community energy systems. In 2017 Australasian Universities Power Engineering Conference (AUPEC) (pp. 1-6). IEEE.

Wang, J., Zhong, H., Ma, Z., Xia, Q., \& Kang, C. (2017b). Review and prospect of integrated demand response in the multi-energy system. Applied Energy, 202, 772-782.

Wang, J., Zong, Y., You, S., \& Træholt, C. (2017c). A review of Danish integrated multi-energy system flexibility options for high wind power penetration. Clean Energy, 1, 23-35.

Wang, T., \& Deng, S. (2019). Multi-period energy procurement policies for smart-grid communities with deferrable demand and supplementary uncertain power supplies. Omega, 89, 212-226.

Warrington, J., Goulart, P., Mariéthoz, S., \& Morari, M. (2013). Policy-based reserves for power systems. IEEE Transactions on Power Systems, 28, 4427-4437. 
Wilson, C., \& Dowlatabadi, H. (2007). Models of decision making and residential energy use. Annual Review of Environment and Resources, 32, 169-203.

Wilson, R. (1977). A bidding model of perfect competition. The Review of Economic Studies, 44, 511-518.

Wolfram, C. D. (1997). Strategic bidding in a multi-unit auction: An empirical analysis of bids to supply electricity. Technical Report National Bureau of Economic Research.

$\mathrm{Xu}$, Y., \& Low, S. H. (2017). An efficient and incentive compatible mechanism for wholesale electricity markets. IEEE Transactions on Smart Grid, 8, 128-138.

Zhang, X., Kamgarpour, M., Georghiou, A., Goulart, P., \& Lygeros, J. (2017). Robust optimal control with adjustable uncertainty sets. Automatica, 75, 249-259.

Zhao, J., Zheng, T., \& Litvinov, E. (2016). A unified framework for defining and measuring flexibility in power system. IEEE Transactions on Power Systems, 31, 339-347.

Zou, P., Chen, Q., Xia, Q., He, C., \& Kang, C. (2015). Incentive compatible pool-based electricity market design and implementation: A bayesian mechanism design approach. Applied Energy, 158, 508-518. 